\title{
Aboriginal marine subsistence foraging flexibility in a dynamic estuarine environment: The late development of Tin Can Inlet (southeast Queensland) middens revisited
}

\author{
Tam Smitha, ${ }^{\mathrm{a}}$ and Ian J. McNiven ${ }^{\mathrm{b}}$ \\ a School of Social Science, The University of Queensland, Brisbane, QLD 4072, Australia; ${ }^{\text {b }}$ ARC Centre of Excellence for \\ Australian Biodiversity and Heritage, Monash Indigenous Studies Centre, Monash University, Clayton, VIC 3800, Australia \\ * Corresponding author tam.smith@uq.edu.au
}

\begin{abstract}
Although the sea arrived in southeast Queensland around 8000 years ago, most estuarine middens date to the past 1000 years. An example is midden deposits dating to the past 400 years forming the upper levels of Sites 62 and $75 \mathrm{~b}$ from Tin Can Inlet located immediately south of the Fraser Island (K'gari) World Heritage Area. Both sites were excavated and analysed in the 1980s. This paper revisits these results following a detailed reanalysis of midden materials and new insights on regional sea level changes. Taking an historical ecology approach, species-specific habitat requirements and associated substrate sediment dynamics help explain similarities and differences between the two midden shell assemblages. Environmental factors and the location of both sites on landforms that formed following sea level fall over the past 2000 years help explain why the basal levels of both sites are probably $<1000-1500$ years old. Documenting pre-2000-year-old Aboriginal use of Tin Can Inlet will need to target more elevated inland dune deposits ( $>5 \mathrm{~m} \mathrm{ASL})$ fronting the mid-Holocene sea level highstand palaeoshoreline.
\end{abstract}

\section{Introduction}

The archaeological record of subtropical southeast Queensland is one of the most intensively studied coastal regions in Australia (Ulm and Hall 1996; McNiven 2006). Research has focused on middens located in two major ecological zones - surf beaches (dominated by Plebidonax (Donax) deltoides middens) and estuaries (dominated by Saccostrea spp., Pyrazus ebeninus and Anadara trapezia middens). Surf beach middens are restricted mostly to the past 1000 years while estuarine middens extend back to 5000 years ago. McNiven $(1989,2006)$ argued that the greater antiquity of estuarine middens may relate to the higher marine resource productivity of estuarine areas compared to surf beach areas. This relatively higher productivity of estuarine areas relates to greater quantities and varieties of marine organisms (mainly shellfish and fish).

For the most part, archaeological research on excavated and radiocarbon-dated estuarine middens in southeast Queensland has focused on Moreton Bay and questions of chronology and the general issue of sea level change (e.g. Ulm and Hall 1996). In some cases, the development of middens dominated by estuarine shellfish and fish has been linked to establishment of nearby estuarine habitats (e.g. Hall 1982, 1999; Walters 1992:176). In other cases, more sociallyoriented arguments have been advanced in situations where development of middens appears to occur hundreds and even thousands of years after establishment of nearby estuarine resources (e.g. Nolan 1986; Walters 1989). To the north of Moreton Bay, social factors have been implicated in late Holocene onset of estuarine middens along the Maroochy River (McNiven 1989) and Tin Can Bay (McNiven 1991a).

In the years since these studies there have been a number of developments in the understanding of estuarine resource habitat dynamics and long-term environmental change both in terms of sea level stabilisation and regional vegetation changes, as well as landscape formation. In the light of these developments, this paper re-examines the estuarine midden Sites 62 and 75b located on the eastern shore of Tin Can Bay (hereafter Tin Can Inlet) immediately south of K'gari (Fraser Island) (Figure 1). These sites were excavated in 1985 by McNiven as part of his PhD research (McNiven 1990a) with results published in McNiven (1991a). The focus of our reexamination is the excavated midden assemblages from both sites which have been housed and curated in the Queensland Museum since McNiven submitted his $\mathrm{PhD}$ thesis in mid1990. Taking an historical ecology perspective, we review, reassess (with additional measurements of molluscan richness and diversity), and recontextualise and reinterpret shellfish assemblages from these sites in light of new understandings of the long-term development of Tin Can Inlet and short-term changes in shellfish habitats. Our findings have implications for modelling long-term and short-term changes in Aboriginal use of marine resources in southeast Queensland over the past 8000 years.

\section{Historical Ecology Perspective}

The theoretical framework for this research is based in historical ecology, providing a vehicle for studying long-term human-environmental relationships. Historical ecology differs from traditional ecology in that it acknowledges the importance of human-environment interactions and utilises deeper historical perspectives, seeing the environment as an outcome of these interactions. Archaeological data such as faunal remains provide information not only on subsistence practices, but also on demography and technology, environmental changes, and human impacts on local habitats and organisms (Braje 2007). Historical ecology allows for the inclusion of evolutionary, ecological, interdisciplinary, and social and historical approaches in interpreting archaeological data. One of its major strengths lies in demonstrating the broader significance of archaeological research to studies of conservation ecology and biology, and cultural and environmental management, as it provides a long-term, historical dataset for the evaluation of human-environmental interactions and the structure of past ecosystems (e.g. Gillies et al. 2018). 


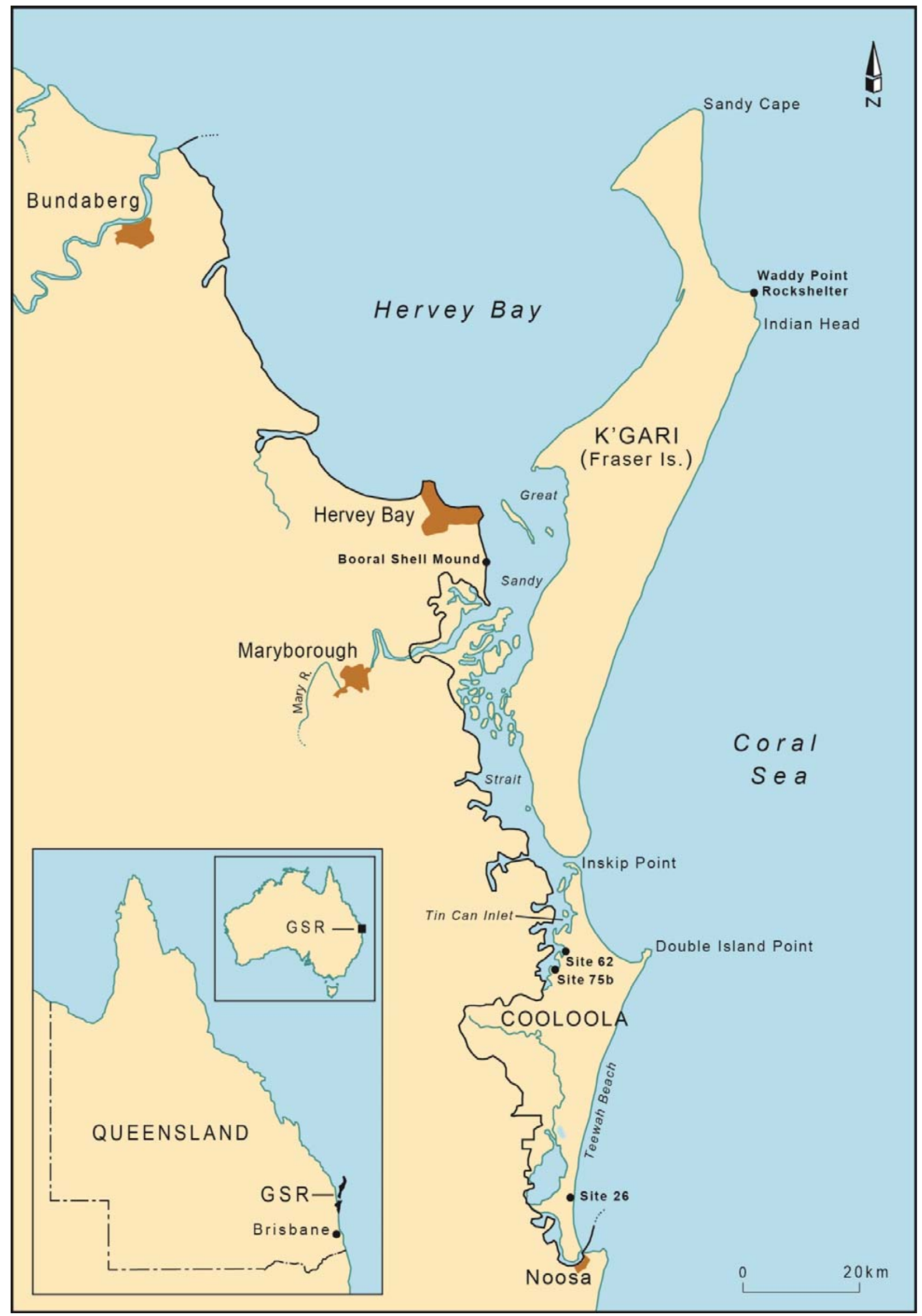

Figure 1. Great Sandy Region (K'gari and Cooloola) showing major towns and archaeological sites mentioned in text. 
The historical ecology perspective is increasingly used internationally in studies of human-environment interaction and the effects of people on ecosystems in a broad range of contexts. Its utility has been demonstrated by Jerardino (1997) and Jerardino et al. (2008) in explicating the deep archaeological record of the South African coast. Similarly, Milner et al. (2007) and Milner (2013) employed the approach to identify human impacts on molluscan assemblages in Orkney and Denmark in the contexts of Viking-age Europe and the Mesolithic-Neolithic transition. The Californian Channel Islands have been the focus of long-term studies (e.g. Ainis et al. 2014; Braje 2007; Braje and Erlandson 2009; Braje et al. 2007, 2011, 2012; Erlandson and Colten 1991; Erlandson and Glassow 1997; Erlandson et al. 1998, 1999, 2004, 2005, 2008, 2009; Rick and Erlandson 2008; Rick et al. 2001, 2005) which encompass not only molluscan studies, but those of marine and terrestrial mammals, and human, faunal, and other environmental relationships to illustrate the changing nature of occupation over a period of 10,000 years. The common theme in these studies is that all humans affect their environment and the larger the population, the larger the potential ecological impacts (Rick and Erlandson 2008). Over extended periods (up to thousands of years) there is the expectation of a wide range of resource harvesting strategies that will differentially alter the environment and ecosystems, requiring 'deft integration of both environmental and cultural information at a variety of temporal and spatial scales' (Crumley 1994:9). This integration requires the differentiation of 'natural' (non-human) ecosystem variations from those caused by humans (Rick and Erlandson 2008) thus introducing ecological and biological information on regional species (faunal and floral) and their responses to natural events (e.g. non-human predation, climate phenomena, and associated impacts such as sea temperature change, disease etc) and changes to the physical environment (e.g. progradation or degradation of landforms).

Thompson (2013:6-7) described historical ecology as an approach that lends itself to the study of small-scale economies (i.e. those that rely on hunting, gathering, fishing, and/or low-level agriculture). This makes historical ecology an ideal basis from which to approach the archaeological record of coastal Australia, and a re-examination of the rich coastal archaeology of southeast Queensland. It also provides the vehicle for the current research to be relevant to global discussions of Holocene human behavioural and environmental relationships.

\section{Tin Can Inlet}

Tin Can Inlet has a tidal hydrology with a central channel (7$11 \mathrm{~m}$ deep) flanked by extensive sand and mud tidal flats forming the southern extension of the Great Sandy Strait (McIntyre 1982:26) (Figures 1-4). It opens to the sea through a $1.0-1.5 \mathrm{~km}$-wide channel between Inskip Point and the southern end of K'gari (Fraser Island). The inlet features a $\sim 60 \mathrm{~km}$-long meandering coastline on its eastern margin extending from Carland Creek in the south to the western tip of Inskip Point in the north. This entire coastline is flat and low-lying with no areas $>5 \mathrm{~m}$ ASL in elevation and most areas $<3 \mathrm{~m}$ ASL. European impacts on the eastern margins of Tin Can Inlet have been minimal and restricted largely to a small log-loading facility at Poverty Point $(1.5 \mathrm{~km}$ northwest of Site $75 \mathrm{~b}$ ) associated with a tramline connecting to logging operations on the Cooloola sandmass in the late nineteenth century (Kerr 1970). Today the area is protected by inclusion in the Cooloola National Park (terrestrial) and the Kauri Creek and Tin Can Inlet Fish Habitat Areas (marine). Various sections of the western margins of the inlet have been impacted by urban development (township of Tin Can Bay established in the 1920s) and a military training area (Brown 2000:186). Loyau (1897:47) stated that the name 'Tin Can' derives from 'Tinkun', the Aboriginal name for the inlet (see also Anonymous 1875). Indeed, in the late nineteenth century the inlet was generally referred to as 'Tincan Bay' by European colonists. According to Watson (1946:114), the 'name is derived from tinchin, or tindhin, the aborigines' name for a species of mangrove'.

\section{Palaeoenvironments and Formation of the Inlet}

The eastern margin of Tin Can Inlet is backed by two physiographic units - a low, $\sim 2.5 \mathrm{~km}$-wide, sandy strand plain across the northern half (tipped by Inskip Point) and a low and undulating coastal sand plain extending up to $\sim 4 \mathrm{~km}$ inland across the southern half (Thompson and Moore 1984). The form of the coastal sand plain 'has been determined largely by the drainage waters issuing from the [Cooloola] sandmass [to the east] and by the marine influences of the inlet [to the west]' (Thompson and Moore 1984:14). Sediments are nearly all podzol and humic podzol sands with small areas of acid peats and derive largely from the Cooloola sandmass. Much of the undulating form of the coastal sand plain was created in the Pleistocene as indicated by a core taken from a lowlying peat swamp (Carlo Creek) located $1.5 \mathrm{~km}$ inland which revealed $\sim 22,000$ year old sandy deposits at a depth of $3 \mathrm{~m}$ (Krull et al. 2004:414). The potential for older Pleistocene landforms across the coastal sand plain is indicated by the interfingering of Cooloola sandmass Dune System 7 dating (OSL) to $\sim 700,000$ years ago across the eastern parts of the plain (Walker et al. 2018). These interfingerings form drainage lines that empty into the inlet indicating that much of the meandering form of the east coast of the inlet is a relic of Pleistocene drainage patterns (Krull et al. 2004:413). The only rock outcrops are low-lying and deeply weathered quartzose sandstone deposits (mostly rubble) at the southern end of the inlet (Grimes 1992a; Thompson and Moore 1984).

Marine processes leading up to the formation of the study area began soon after 20,000 years ago when sea levels began rising with global warming at the end of the last Ice Age and the $70 \mathrm{~km}$-wide continental shelf east of Inskip Point flooded rapidly at an average rate of nearly $250 \mathrm{~m}$ per generation between 15,000 and 8000 years ago (Hill 1992:Figure 1; Williams et al. 2018:Figure 2). Just prior to 8000 years ago, advancing sea began moving further inland to drown the lowlying river valley behind the high sandmasses of K'gari (to form Hervey Bay and Great Sandy Strait) and Cooloola (to form Tin Can Inlet) (Grimes 1992a:32-33; Ward and Grimes 1987; see also Lewis et al. 2013). It appears that K'gari was cut off from the mainland by rising seas running up palaeochannels (now submerged) of the Mary River drainage system located to the north of the island (Payenberg et al. 2006) and possibly at the south end of the island (Grimes 1992a:5, 32; McIntyre 1982:156, 175-176, 201).

A range of evidence from the Great Barrier Reef indicates that rising sea levels reached current levels $\sim 8000$ years ago and continued rising a further $1.0-1.5 \mathrm{~m}$ which were maintained between $\sim 7000$ and $\sim 4000$ years ago and then, with some fluctuations, fell back to modern levels, especially 


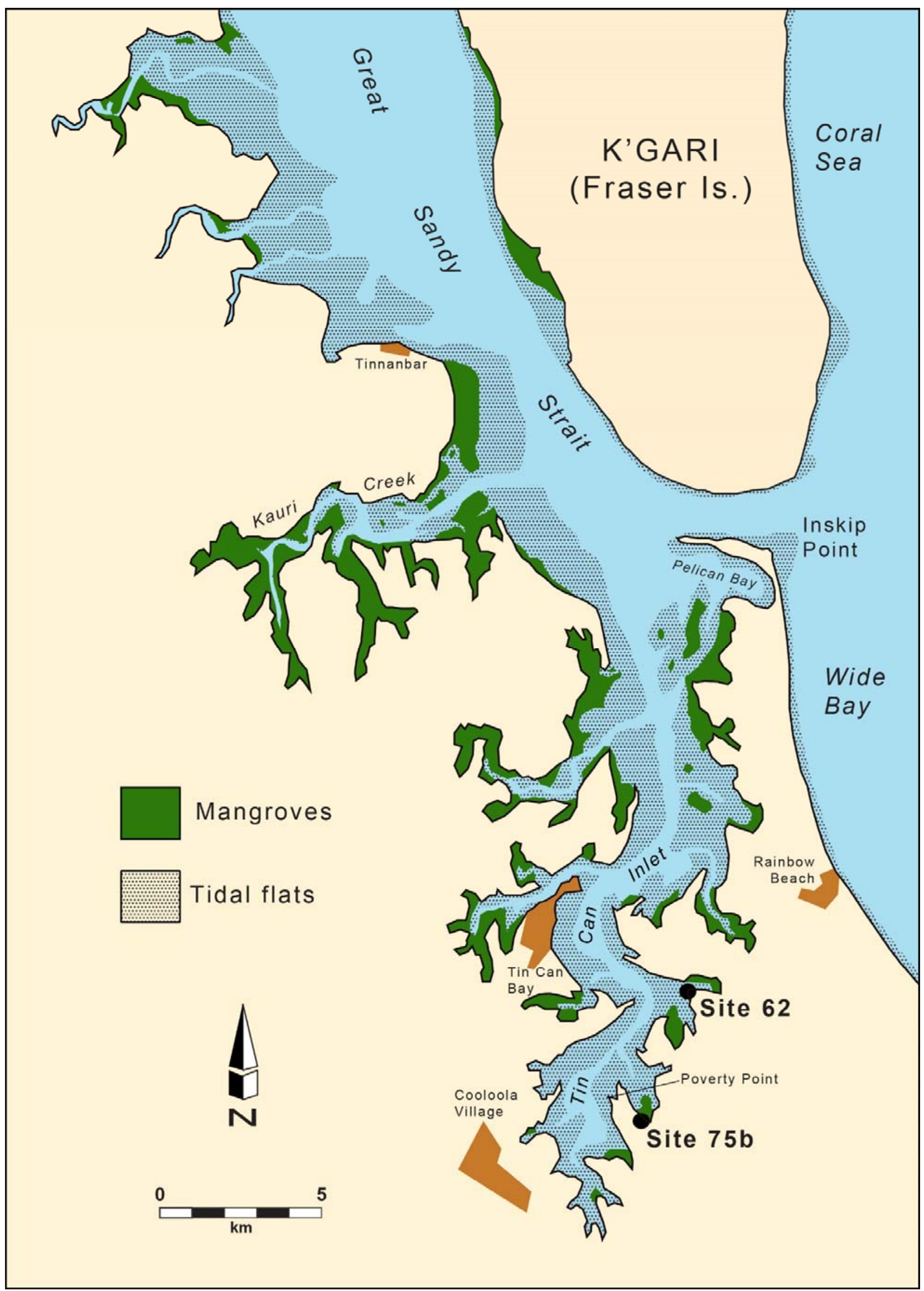

Figure 2. Intertidal flats and mangroves in the study area during the 1970s (after Dredge et al. 1977:Figures 2-3) with contemporary townships. 


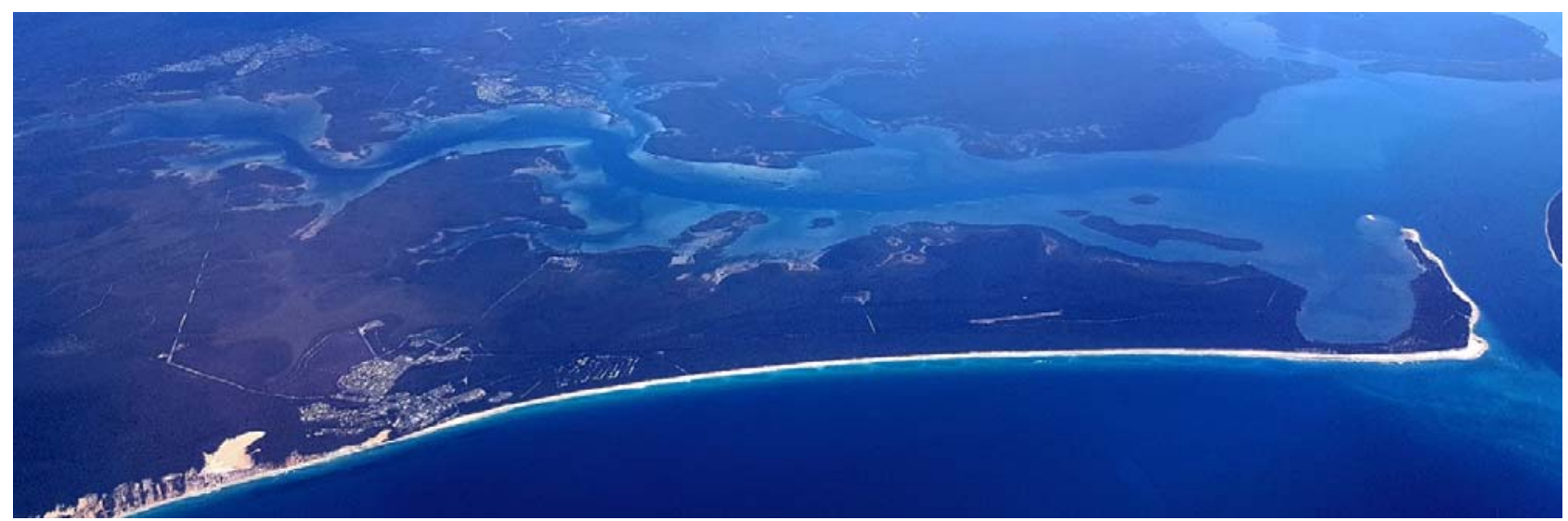

Figure 3. Oblique aerial photo of Tin Can Inlet with Rainbow Beach township (lower left) and southern tip of K'gari (far centre right), looking west (Photograph: Ian J. McNiven, August 2018).

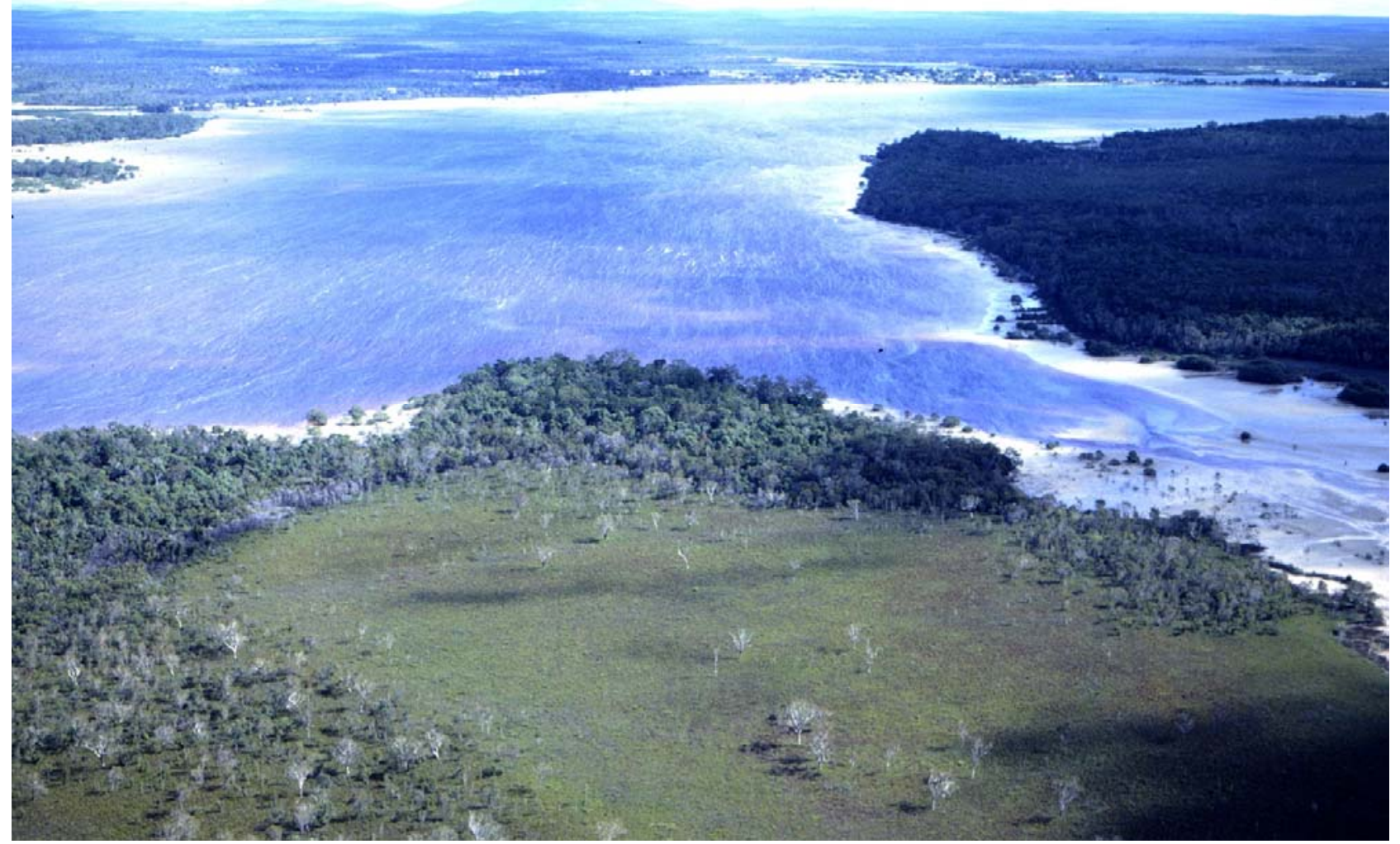

Figure 4. Oblique aerial photo of the central sections of Tin Can Inlet looking northwest over Cameron Point and Site 62 (centre), looking towards Tin Can Bay township (Photograph: Ian J. McNiven, July 1985).

over the past 1000 years (Lewis et al. 2013:126-127; see also Sloss et al. 2018). In Moreton Bay in southeast Queensland, sea level appears to have been at least $1.1 \mathrm{~m}$ higher between $>$ c. 6700 and c. 5700 years ago, after which it started to fall to modern levels (Cooley 2017:122; Leonard et al. 2013; Major 2012), possibly mostly within the past 2000 years as indicated by progradation of beach ridges (Cotter 1996; Ward and Hacker 2006). Similar coastal progradation of low-lying sand dune ridges $<5 \mathrm{~m}$ above sea level over the past 2000 years has been documented for the mainland coast of Hervey Bay (QBPA 1989).
The most direct implications of the mid-Holocene sea level highstand is that essentially the entire eastern terrestrial margin of Tin Can Inlet would have been inundated by the sea at this time such that the inlet would have been wider and larger in area (see Moss et al. 2013:44). Indeed, most of the $4 \mathrm{~km}$-long recurved spit of Inskip Point, forming Pelican Bay, probably only formed within the past 2000 years (see McIntyre 1982:155, 173, 182). 'Patches of slightly higher sandy sheets and low sandy banks occur sporadically along the coastal margin of the plain and probably represent winddrifted sand from the intertidal zone' (Thompson and Moore 
1984:14). More elevated areas of higher ground (5-10m ASL) are currently located hundreds of metres inland and would have been suitable for Aboriginal camping for the past 8000 years. In marked contrast, the entire eastern margin of the inlet most likely formed (and became available for camping) only within the past 1000-2000 years as sea levels dropped to modern levels.

A range of evidence indicates that estuarine habitats for marine shellfish and fish were present in the broader region since arrival of the sea $\sim 8000$ years ago. Coring of fens at Moon Point on the northwest side of K'gari reveals mangrove pollen dating back to $\sim 5000$ and perhaps $\sim 10,000$ years ago (Moss et al. 2013:26, 2016:822), while coring of fens of the Cooloola sand plain backing eastern Tin Can Inlet reveals mangrove pollen dating back to $\sim 7000-8000$ years ago (Moss et al. 2013:39-40; Withers 2013:60,63). The earliest known evidence for marine shellfish in Tin Can Inlet is a calibrated radiocarbon date of $\sim 6800$ years on 'shells' obtained from a core taken at Bullock Point on the southwest corner of Pelican Bay (Figure 2) (Grimes 1992b:26; Ward and Grimes 1987:329). More secure evidence comes from Booral Shell Mound located $50 \mathrm{~km}$ north of Tin Can Inlet on the mainland coast of Great Sandy Strait. This archaeological site contains estuarine shellfish (e.g. oyster, Saccostrea spp.) and crustaceans (e.g. mud crab, Scylla serrata) dating back to 3000 years ago (Bowen 1998; Frankland 1990; McNiven 1994; Smith 2016). More broadly, a diverse marine shell deposit (e.g. A. trapezia, P. ebeninus, Saccostrea spp.) with an associated calibrated radiocarbon date of $\sim 8300$ years ago on the Maroochy River located $70 \mathrm{~km}$ south of Tin Can Inlet supports the view that estuarine shellfish resources have been available in the Great Sandy Region since arrival of the sea (Wood 1972; Wood and King 1974). Similarly, Hofmann (1980:508) reports estuarine sediments with 'traces of shells' associated with the North Pine River of Moreton Bay (located $145 \mathrm{~km}$ south of Tin Can Inlet) with an associated calibrated radiocarbon date of $\sim 9300$ years ago. As expected, the early Holocene estuarine deposits of the Maroochy and North Pine Rivers occur $5.2 \mathrm{~m}$ and $7.5 \mathrm{~m}$ below current sea level and relate to the final stage of the marine transgression (see Lewis et al. 2013).

Freshwater is freely available throughout the year from swampy depressions across the coastal sand plain and numerous small spring-fed creeks (Reeve et al. 1985). It is likely that groundwater became increasingly available across the Cooloola sand plain backing Tin Can Inlet as the water table rose in response to sea level rise $\sim 8000$ years ago and wetlands, especially in the form of shallow lakes, expanded (Krull et al. 2004; Moss et al. 2013:44, 2016). As sea level fell after the mid-Holocene highstand, these lake wetlands transformed into swampy wetlands and fens, especially over the past 1000 years (Moss et al. 2013:42-43; Withers 2013:74, 80).

The mid-Holocene sea level highstand coincided broadly with warmer temperatures and variable wetter/drier conditions in the Great Sandy Region as revealed by pollen cores taken from perched lakes on K'gari. For example, Lake Jennings reveals evidence of warmer temperatures and increased effective precipitation relative to modern levels between $\sim 6000$ and $\sim 3500$ years ago (Hembrow et al. 2018:564). In contrast, drier conditions are registered at Lake McKenzie between $\sim 8000$ and $\sim 4000$ years ago (Atahan et al. 2015:152-153; Hembrow et al. 2014:782; Woltering et al.
2014:142), at Lake Allom between $\sim 12,000$ and $\sim 5400$ years ago (Donders et al. 2006:433, 435), and at Hidden Lake between $\sim 8500$ and $\sim 2500$ years ago (Longmore 1998). That wetter and warmer conditions were likely to be more pervasive across the region during the mid-Holocene is in keeping with increased moisture between $\sim 7000$ and $\sim 4000$ years ago on North Stradbroke Island (Barr et al. 2013) and warmer and wetter conditions more generally across eastern Australia during the mid-Holocene $\sim 8000-5000$ years ago (Reeves et al. 2013:29). Over the past $\sim 3500$ years, reduced effective precipitation approaching modern climatic patterns is recorded at Lake Jennings (Hembrow et al. 2018:565) and Lake Allom (Donders et al. 2006:436).

The upshot of these environmental changes is that Tin Can Inlet has been suitable for coastal occupation by Aboriginal people since arrival of the sea $\sim 8000$ years ago due to the availability of freshwater swamp resources (e.g. drinking water and plant foods) and marine resources (e.g. shellfish and fish). However, the eastern margins of Tin Can Inlet elevated $<1.5 \mathrm{~m}$ ASL only become available for Aboriginal camping as 'new landscapes' within the past 1000-2000 years as sea levels fell to modern levels after the mid-Holocene highstand of $+1.0-1.5 \mathrm{~m}$. The best opportunities for finding archaeological evidence of older (pre-2000 year old) Aboriginal settlement along Tin Can Inlet will be higher dune deposits ( $>5 \mathrm{~m}$ ASL) located along the mid-Holocene palaeoshoreline located up to $1-2 \mathrm{~km}$ inland (see Cotter 1996; Hall 1999:178).

\section{Aboriginal Associations}

According to Harry Aldridge who lived on the mainland coast of Great Sandy Strait during the late nineteenth century, 'Tin can' was the territory of the 'Paringnoba' who may have been a 'tribe' or one of a number of 'tribelets or clans of one great tribe' (Howitt 1904:58-59). In terms of more encompassing tribal designations, amateur anthropologist John Mathew (1910:map facing pp.67, 108) included Tin Can Inlet within the huge territory of the 'Kabi tribe', adding that 'coastal' peoples were known as the 'Bīdhala' (Mathew 1910:108). Anthropologist Norman Tindale (1974:SE map) included Tin Can Inlet within the large tribal territory of the 'Batjala' which included K'gari and adjacent mainland areas. More specifically, Tindale (1974:165) saw Tin Can Inlet, along with Cooloola and the southern third of K'gari, as belonging to the 'Dulingbara' 'horde groups' of the Batjala who 'behaved much as if a separate tribe'. The anthropological notion of Tin Can Inlet as part of the lands of the Butchulla people is supported by contemporary Aboriginal oral tradition and law. For example, Reeves and Miller (1964:3) state explicitly that 'Butchulla territory was Fraser Island, Double Island Point, Tin Can Bay, Bauple Mountain and north to a point at the Burrum Heads' (see also Miller 1993). Foley (1994:7, map) similarly notes that 'Badtjala traditional homelands' include K'gari and adjacent mainland areas that take in northern Cooloola (encompassing Tin Can Inlet and Double Island Point). The upshot of these affiliations is that ethnographic and ethnohistorical information relating to $\mathrm{K}$ 'gari and Great Sandy Strait is also relevant for Tin Can Inlet.

Little historical information is available on European sightings of Aboriginal people along the eastern margins of Tin Can Inlet during the nineteenth century. Through their connections with neighbouring coastal groups, Aboriginal peoples of Tin Can Inlet would have been aware of visits to 
the coasts of Cooloola and K'gari by European explorers, mariners, castaways, and escaped convicts between 1800 and 1840. They also would have been aware of escalating violence and massacres that accompanied European colonial settlement of the region during the 1850 s, especially adjacent inland regions of the pastoral frontier (Evans and Walker 1977). Numerous Aboriginal people involved in frontier resistance raids were known to take safe refuge on $\mathrm{K}$ 'gari (Fraser Island), and it is probable that other coastal sand dune regions such as Tin Can Inlet and Cooloola similarly functioned as frontier 'refuges' (Evans and Walker 1977:52, 56; Heap 1966:10; McNiven 1991a:103, 2006:118-120).

As frontier dynamics moved from overt resistance and violence in the $1850 \mathrm{~s}$ to covert resistance and strategic accommodation and adaptation in the 1860s, Aboriginal peoples of the Tin Can Inlet (and affiliated neighbours) became involved in the rapidly developing colonial economy such as the timber industry of northern Cooloola and the dugong fishery and oyster farming of the inlet and Great Sandy Strait. Aboriginal involvement in logging operations across the high dunes of northern Cooloola appear to have spanned the 1860s-1880s (Brown 2000:146-154). William Pettigrew, who owned these operations, was shown the location of the commercial stands of Kauri pine by Aboriginal guides in the 1860s (Brown 2004:153,160; C. Petrie 1904:187; W. Petrie 1921:5). The critical role of Aboriginal people in the Tin Can Inlet dugong fishery may have commenced in the $1850 \mathrm{~s}$ and is well-documented for the 1860s and 1870s (Anonymous 1876; Brown 2000:170; Daley et al. 2008:233; Lavers 1871; Thorne 1876:249). Aboriginal people were also involved in the Tin Can Inlet oyster (Saccostrea spp.) industry during the 1870 s and through into the 20th century (Anonymous 1877; Brown 2000:173; Devoy 1902:20; Fison 1889; Frawley 2017:12; Smith 1981:50, 52; Woolley 2016).

The closest and most consistent recordings of Aboriginal people on the eastern shores of Tin Can Inlet, dating from the 1820 s through to the $1880 \mathrm{~s}$, are of what appears to have been a permanent or semi-permanent campsite at nearby Inskip Point on the mainland opposite the southern end of K'gari (Anonymous 1870; Brown 2000:67-68; McNiven 1990a:5680 ; Russell 1845:314, 1888:260-264). In the 1880s, Aboriginal people at Inskip Point were provisioned annually with government blankets (e.g. Anonymous 1880, 1888). In 1896, Archibald Meston visited Inskip Point as a potential location for an Aboriginal mission which eventually was established at Bogimbah Creek on the west coast of K'gari (Anonymous 1896). The establishment of the mission marks the end of available information on Aboriginal settlement of eastern Tin Can Inlet. As such, archaeological evidence for post-contact occupation and use of the eastern shores of the inlet may extend from the 1850 s through to the 1890 s.

Remarkably, the only known nineteenth century images of Aboriginal people along the east coast of Tin Can Inlet are two photographs taken at the launch of the Mary Ann steam engine and railway line near Poverty Point associated with Pettigrew and Sim's Cooloola forests timber-getting operations in October 1873 (Brown 1999; Kerr 1970) (Figures $2,5)$. The photographs show at least six Aboriginal men standing in the background, some in trees, who were probably employed (Brown 1999:244; Elaine Brown, pers. comm., 2018) on low wages and also paid in kind with tea, flour, tobacco, clay pipes etc (see Evans and Walker 1977:61-66).

\section{Aboriginal Food Resources}

Available ethnographic and historical information suggests strongly that Aboriginal subsistence practices in the Tin Can Inlet region (K'gari and Cooloola) during the nineteenth century focused on plants from swamps and rainforests and marine fish and shellfish (see Devitt 1979; McNiven 1992a for overviews). To date, few nineteenth century historical records have been found specifically documenting Aboriginal activities in Tin Can Inlet.

\section{Plants}

The coastal fringe vegetation of Tin Can Inlet is influenced by local drainage regimes and varies from tall open forests on higher elevation areas to swamp communities in the lower depressions. Four vegetation zones have been identified for the area: forest red gum (Eucalyptus tereticornis) and cypress (Callitris columellaris) forest complexes, and casuarina (Casuarina littoralis) and Queensland brush box (Lophostemon confertus, syn. Tristania conferta) forests; wallum banksia (Banksia aemula) woodland and scribbly gum (Eucalyptus signata) woodland; heathland (Banksia spp.) ranging to paperbark (Melaleuca quinquenervia) swamps, mixed paperbark woodland, and paperbark and forest red gum woodland; and large mangrove forests (e.g. orange mangrove, Bruguiera gymnorrhiza; grey mangrove, Avicennia marina; and spotted mangrove, Rhizophora stylosa) in the intertidal zones of most of the inlet. Major plant food sources include bungwall or swamp fern (Blechnum indicum), cabbage-tree palm (Livistonia decipiens), bracken fern (Pteridium esculentum), and orange mangrove (Bruinsma and Danaher 2000; Fisheries Research Consultants 1993; Harrold et al. 1987; Sandercoe 1986; Thompson and Moore 1984).

\section{Animals}

The rich marine resources available within Tin Can Inlet include 39 species of fish of modern commercial or recreational importance, with bream (Acanthopagrus spp.), whiting (Sillago spp.), flathead (Platycephalus spp.), and mullet (Mugil spp.) constituting major elements of the shallow water fishery (Beumer and Halliday 1994:2; Morton and Healy 1992:14). The sand banks, mudflats, and seagrass and mangrove communities within the intertidal zone support a wide variety of molluscan species such as oyster (Saccostrea spp.), Hercules club whelk (P. ebeninus), cockle (A. trapezia), and nerites (Nerita spp.), crustaceans such as mud crab ( $S$. serrata) and sand crab (Portunus pelagicus), and a nursery environment for juvenile prawns (Fisheries Research Consultants 1993, 1994). Loyau (1897:52) noted that 'Rock oysters abound in the mangrove lined inlets of Hervey's and Tin Can bays, and all other sea delicacies are plentiful'. Meston (1895:140) proclaimed that 'Tin Can Bay ... is a famous place for oysters'. The high productivity of oysters in the region is indicated by the presence of numerous commercial oyster operations within Great Sandy Strait and Tin Can Inlet over the past 150 years (Fison 1889; Morton and Healy 1992:15; Woolley 2016). Tin Can Inlet also supports an active mud crab industry (Dredge et al. 1977:8; Morton and Healy 1992). 


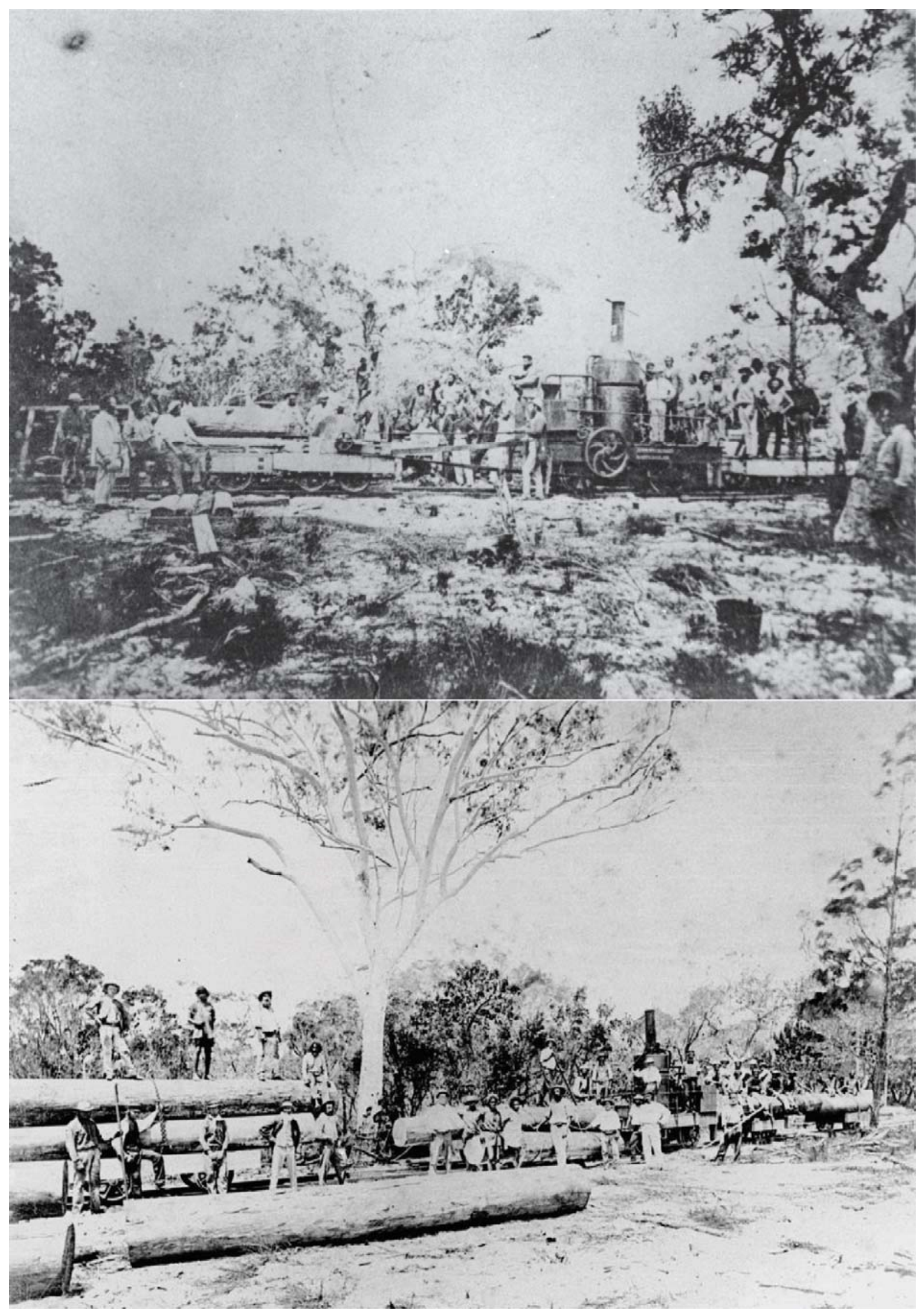

Figure 5. Photographs of Aboriginal people participating in the official opening of the Kaloola railway associated with timber-getting operations of Pettigrew and Sim located near Poverty Point, southeast Tin Can Inlet, in October 1873. Photographs by Edward Forster from the Maryborough Chronicle newspaper (Source: John Oxley Library). 
Great Sandy Strait and to a lesser extent Tin Can Inlet also support turtles (e.g. green sea turtle, Chelonia mydas, hawksbill turtle, Eretmochelys imbricata) and a range of marine mammals such as dugongs (Dugong dugon), dolphins (e.g. Indo-Pacific humpback dolphins, Sousa chinensis), and humpback whales (Megaptera novaeangliae) (Cagnazzi et al. 2011; Corkeron 1995; Fisheries Research Consultants 1993:28; Preen and Marsh 1995). Of dugongs it was observed that they 'are found in immense numbers in Tincan Bay' (Anonymous 1873:3). Turtles were said to 'abound in the [Tin Can Inlet] Bay' (Anonymous 1873:3).

By comparison, terrestrial faunal resources flanking Tin Can Inlet are depauperate in terms of species diversity and abundance. Barry and Campbell (1979:173) remarked on the 'high diversity' of mammals and amphibians on the Cooloola Peninsula, but other sources (Dwyer et al. 1979a, 1979b) suggest that the potential terrestrial mammal resource base of the coastal and strand plains is poor. Although Barry and Campbell (1979) recorded a number of amphibian species for the Cooloola peninsula, reptile records for the general area are poor to non-existent.

\section{Aboriginal Cultural Sites}

Over 70 Aboriginal cultural sites have been recorded along the eastern margin of Tin Can Inlet. These include ceremonial bora rings (associated with intergroup gatherings and initiations - see Satterthwait and Heather 1987), and middens, stone artefact scatters, and a scarred tree (McNiven 1984:85, 1985:14, 1990a:188, 1991a:87). Two bora rings are reputed to exist in the southeast sections of Tin Can Inlet while a large and well-preserved bora ring is located within Pipeclay National Park located in the southwest corner of the inlet (Steele 1983:186). Most middens are $<10 \mathrm{~cm}$ thick and can be seen eroding from a low (1.0-1.5m-high) erosion face which flanks most of the eastern margins of the bay at the high water mark (for further details see McNiven 1991a). Nearly all middens $(91.3 \%)$ are located within forest red gum and cypress forest, Casuarina and Queensland brush box forest, and scribbly gum woodland (McNiven 1985:15, 1991a:87, 101; see Smith 1992; Stockton 1974 for similar findings from the Moreton Bay Region). A wide range of stone artefact raw materials was found associated with middens, including those sourced locally (e.g. Double Island Point andesite) and from the hinterland (e.g. silcrete, quartzite, chert). The most common formal stone artefact implement type was beveledged tools, a well-known tool type from coastal southeast Queensland used to process plant foods (e.g. Gillieson and Hall 1982; Kamminga 1981; McNiven 1992b). As only small estuarine midden scatters largely devoid of stone artefacts were found along swamp margins up to $7 \mathrm{~km}$ inland across the adjacent coastal sand plain, McNiven (1992c) hypothesised that the relatively large middens located along the shoreline of eastern Tin Can Inlet functioned as home bases where people processed and consumed marine foods obtained from the inlet and plant foods obtained from adjacent inland swamps. This settlement-subsistence pattern was associated with Recent Phase occupation of the broader Cooloola region over the past 900 years which saw establishment of a separate coastal residential group in the region (McNiven 1991b, 1999). Excavations at Tin Can Inlet midden Sites 62 and 75b provided artefactual and chronological data that contributed to defining the Recent Phase.

\section{Analytical Methods}

Central to the aims of this study is the use of archaeological molluscan remains as indicators both of human behaviour and environmental variability. To this end, a range of analyses was employed on the molluscan material beyond the 'traditional' quantitative measures. The analyses, and their purposes, are outlined in this section.

\section{Weight, NISP and MNI}

Each taxon was weighed and the numbers of identifiable specimens (NISP) and minimum number of individuals (MNI) counted. While there are ongoing debates about the most appropriate method for quantifying molluscan remains (e.g. Claassen 1998, 2000; Giovas 2009; Glassow 2000; Lyman 2008; Mannino and Thomas 2001; Mason et al. 1998; Nunn et al. 2007; Reitz and Wing 2008), this combination is frequently used in faunal studies, as it is more able to accurately describe the proportions of taxa, the degree of intersite and intrasite fragmentation, and also allows comparisons with non-molluscan components (e.g. Faulkner 2013:73; Szabó 2009; Ulm 2006a).

The use of NISP and MNI also allowed for calculation of the fragmentation ratio (NISP:MNI) for individual taxa, which may reflect the level of intensity of site use, while acknowledging morphological differences between taxa that may affect fragmentation rates (e.g. thin vs. thick, obese vs. flat valves), as well as micro-structural differences that can assist particular taxa to resist crushing or treadage. Sites at which deposition or accumulation rates are low and where cultural material, including shell, is exposed for longer periods before burial, generally exhibit greater rates of fragmentation than those sites where deposition is more rapid and cultural material is buried before prolonged exposure to weathering and treadage (Claassen 1998; Faulkner 2010, 2013; Hiscock 1985).

\section{Morphometric Analyses}

A range of measurements was taken, including valve height, length and depth, hinge length, and umbo height and length for bivalves; and shell height, width, spire height, aperture width and aperture height for gastropods. These morphometric data are important in determining the composition of samples in terms of population structure, determining ratios of juveniles to adults, levels of exploitation and potential evidence of resource depletion, and biological, ecological, and environmental conditions (see e.g. Claassen 1998; Faulkner 2006, 2008, 2013; Giovas et al. 2010, 2013; Szabó 2009). Breakage patterns were also noted, as was the condition of the shell (e.g. chalky, weathered, degraded, burned, or damaged by worms or borers) (see Zuschin and Stanton 2001).

\section{Statistical Analyses}

The degree of species richness, diversity, and evenness in the assemblages was measured. The characteristics and performance of the diversity statistics vary in terms of their discriminant ability, sensitivity to sample size, and focus on richness or evenness/dominance. NTAXA was used to assess species richness. Shannon's Index of Diversity $(H)$ and Shannon's Evenness (E) were used to measure evenness (relative abundance of species). $H$ values range between 0 and 
5 - the higher the $H$ value, the greater species diversity and richness. E values range between 0 and 1, with a value of 1 indicating that all taxa are equally abundant. Simpson's Index of Diversity $(1-D)$ with values from 0 to 1 was used to determine the dominance of few species in the assemblage, with values close to zero indicating dominance by a single taxon (Harris et al. 2015; Lyman 2008; Magurran 2004). All diversity indices were calculated using Palaeontological Statistics (PAST) version 3.04 (Hammer et al. 2001).

Additional statistical analyses were also performed on the morphometric data and varied according to the assumptions being tested. The appropriate tests were determined by reference to Field (2014) and Pallant (2013), and the analyses undertaken using IBMC SPSSC Statistics Version 22.

\section{Tin Can Inlet Midden Site 75b}

Site $75 \mathrm{~b}$ is the more southerly of the two sites and is located $1.5 \mathrm{~km}$ southeast of Poverty Point (Figure 2). It is part of a large complex of middens located along a small baylet adjacent to extensive tidal mudflats, mangroves, and $\sim 20 \mathrm{~m}$ from a freshwater spring (for details see McNiven 1990a, 1991a). The midden was intermittently exposed over at least $10 \mathrm{~m}$ within a mixed Melaleuca, Eucalyptus, Banksia, and cypress forest (Figure 6). Two contiguous $50 \mathrm{~cm} \mathrm{x} 50 \mathrm{~cm}$ excavation pits (Squares A and B) were placed in an area of relatively dense surface midden deposit. The surface of the excavation pit is elevated only $1.2 \mathrm{~m}$ above the high water mark. Squares A and B were excavated using 21 Excavation Units (XUs) down to depths of $92 \mathrm{~cm}\left(0.23 \mathrm{~m}^{3}\right)$ and $89 \mathrm{~cm}$ $\left(0.22 \mathrm{~m}^{3}\right)$ respectively between 6 and 9 December 1985 . Excavated sediments were wet-sieved through $3 \mathrm{~mm}$ mesh. Two major stratigraphic units, SU1 and SU2, were identified (Figures 7-8). SU1 was further divided into subunits 1a and 1b: SU1a consisted of relatively loose dark grey sand that was slightly acidic ( $\mathrm{pH}$ value 4.5 ), while SU1b consisted of light brownish grey sand of a more neutral $\mathrm{pH}$ value (7.5) and contained a concentration of shell, as well as the majority of the other cultural material recovered. SU1 varied in thickness from $9 \mathrm{~cm}$ to $23 \mathrm{~cm}$. SU2 was loose sand grading with depth from light grey to white and very light grey with $\mathrm{pH}$ values ranging from 8.0 in the upper levels to 6.5 at the base. It contained a low number of shell fragments and stone artefacts in the context of large tree roots.

Due to time restrictions, only faunal materials (molluscan and vertebrate remains) from Square A (which contained the bulk of midden material recovered from the pit) were fully analysed by McNiven (1990a, 1991a). The data presented for the molluscan remains consisted of MNI for each taxa and total shell weights per XU. McNiven obtained a single radiocarbon date for the site based on a mixed sample of oyster (Saccostrea spp.) and Hercules club whelk ( $P$. ebeninus) from XU7 (depth $=18-21 \mathrm{~cm}$ ) at the base of the main midden deposit (SU1b) in Square A. McNiven reported a conventional age of $700 \pm 70$ BP (Beta-19421), with a calibrated age (corrected for marine reservoir effect) of 'modern' (i.e. nineteenth century). We update this calibration using the online calibration program Calib 7.10 (Stuiver and Reimer 1993) and the marine calibration dataset (Marine13) (Reimer et al. 2013), along with the northeast Australia regional $\Delta \mathrm{R}$ correction value of $12 \pm 10$ (Ulm 2006b; see also Ulm 2002a and Ulm et al. 2009 for a discussion of the complexities of determining $\Delta \mathrm{R}$ values for estuarine shell). The revised calibrated date is $147-479$ cal BP $(95.4 \%$ probability) with a median age of $340 \mathrm{cal}$ BP (Table 1). As such, the main midden deposit at Site $75 \mathrm{~b}$ spans a few hundred years, possibly from the fifteenth century to the end of the nineteenth century when traditional Aboriginal use of northern Cooloola ceased due to the devastating impacts of European colonial invasion (McNiven 1990a, 1991c:13). The presence of $\sim 5 \mathrm{~cm}$ of sandy sediments with a low density of cultural remains above the main midden deposit (Figure 8) possibly reflects continued sedimentation at the site after Aboriginal occupation ceased. The basal levels of the site with low numbers of stone artefacts probably date to $<1000-1500$ years ago based on radiocarbon dating of Site 62 (see below).

\section{Molluscs}

Square A

A total of $6656.2 \mathrm{~g}$ of marine shell comprising 14 taxa with a MNI of 909 was recovered from Square A (Tables 2-3). The shell was most numerous in the upper eight XUs, after which numbers declined significantly with only a few specimens identified and quantified by MNI below that level, although shell weights were recorded for all but one XU (Table 2). McNiven noted that all the taxa except for P. deltoides (pipi) could be obtained from the intertidal zone adjacent to the site. The pipi were available from Rainbow Beach and/or Teewah Beach at a distance of $9 \mathrm{~km}$ and $13 \mathrm{~km}$ respectively. Also noted were vertical changes in the distribution of the molluscan species: while Saccostrea spp. (oyster) and P. ebeninus (Hercules club whelk) occurred throughout most of the midden, Isognomon spp. (toothed or tree oysters) occurred only in the top four XUs, and Conuber sordidum (sand snails) occurred only in XUs 4-7. Possible reasons for these variations are discussed below.

\section{Square B}

Smith (2016) sorted and analysed the faunal component of Square B. This assemblage was from the eastern periphery of the major midden deposit and exhibited only slight differences in composition compared to Square A. Unlike Square A, shell was only recovered in the upper seven XUs of Square B (Table 4). Species recorded in Square A but absent from Square B were Bedeva paivae (oyster drill), Patelloida mimula (limpet), Ergalatax contracta (murex), and Cerithidea largillierti (mud whelk). Likewise, two taxa recorded in Square B were absent from Square A: ceriths (creepers, Cerithium spp.) and false or yellow striped mussel (Fluviolanatus subtorta). For the Isognomon spp. and Cerithium spp. insufficient landmarks were preserved for confident identification beyond genera. The total NISP for molluscs in Square B was 4386 (Table 5). At 348 the total MNI for Square B was fewer than that recorded for Square A, reflecting the less dense concentration of midden deposit in the square (Table 6). Estimates of MNI based on volume are $3952 / \mathrm{m}^{3}$ for Square A and $1513 / \mathrm{m}^{3}$ for Square B (Table 7). Shell weights were similarly lower in Square B (2779.28g) than those recorded for Square A (Tables 4, 8).

Although there is a difference in scale, both squares exhibit similar trends in shell discard. Saccostrea spp. discard peaks in both squares at XU4, and shell weight is highest in XUs 3 to 5 in both squares (Tables 2, 3,7). Measures of richness, evenness, and diversity reflect low-to-moderate species diversity and richness, and the dominance of Saccostrea spp. in the assemblages, albeit less marked for Square B (Tables 9-10). 


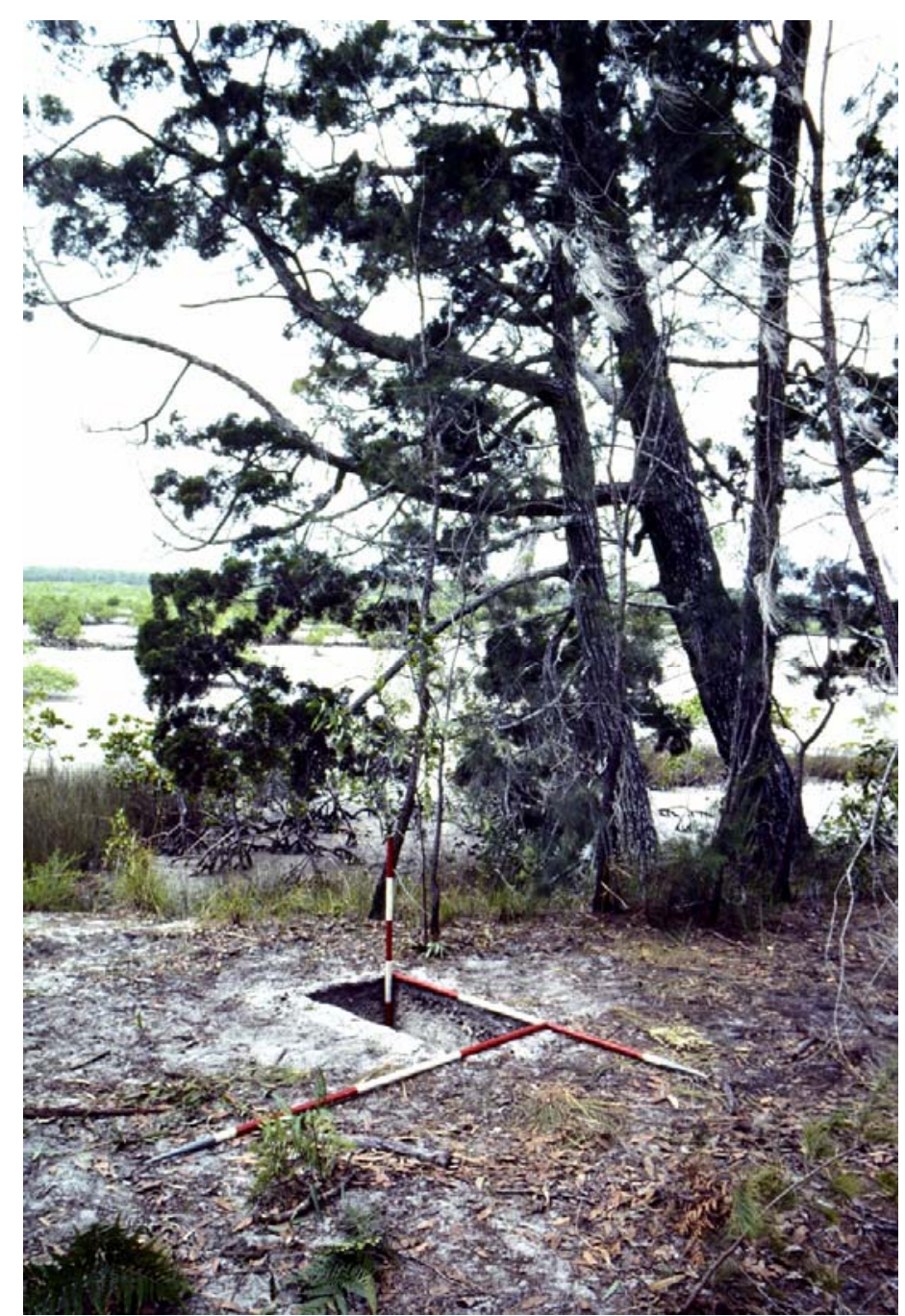

Figure 6. Site 75b after excavation, looking northeast (Photograph: Ian J. McNiven, December 1985).

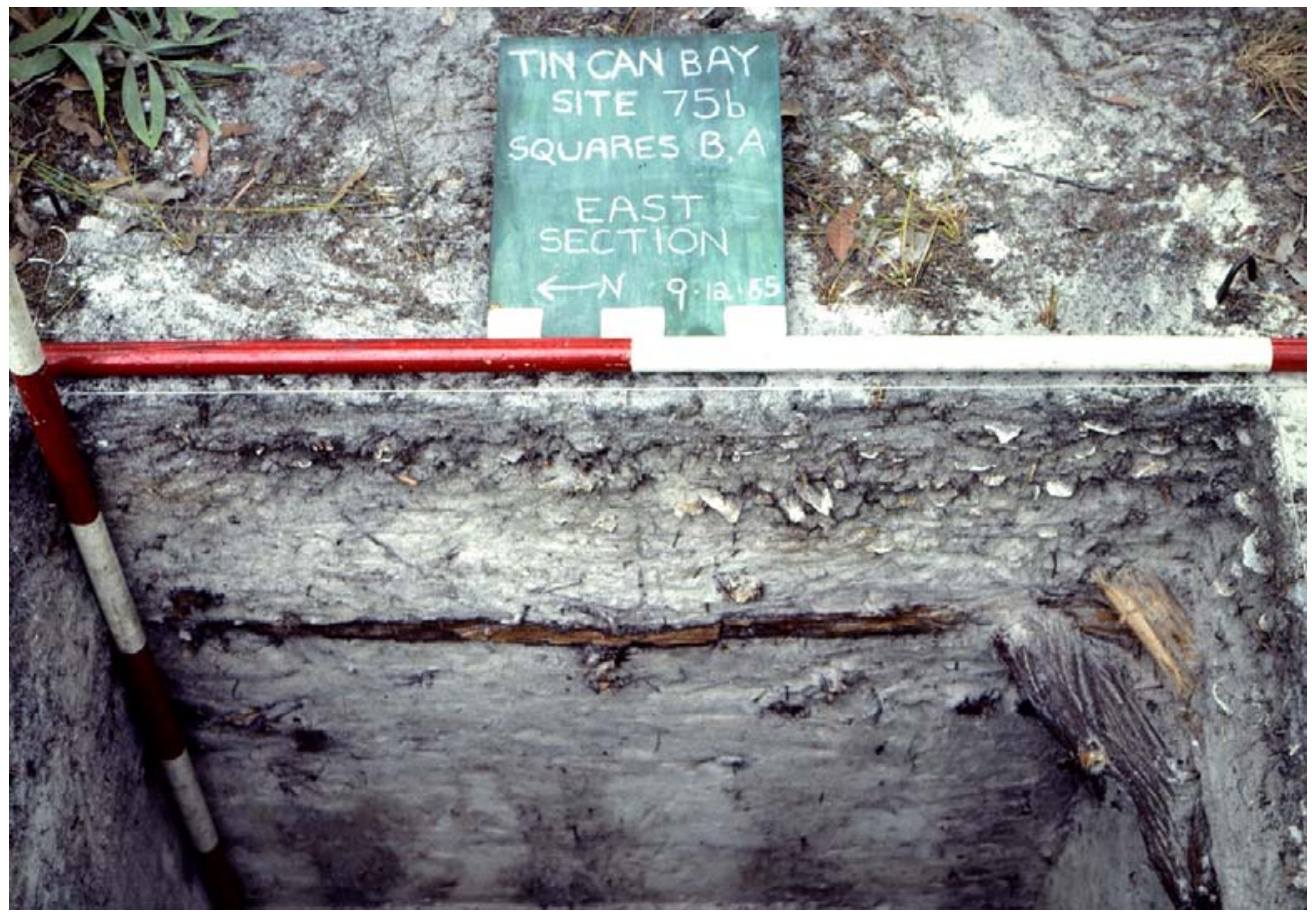

Figure 7. East section of Site 75b after excavation (Photograph: Ian J. McNiven, December 1985). 


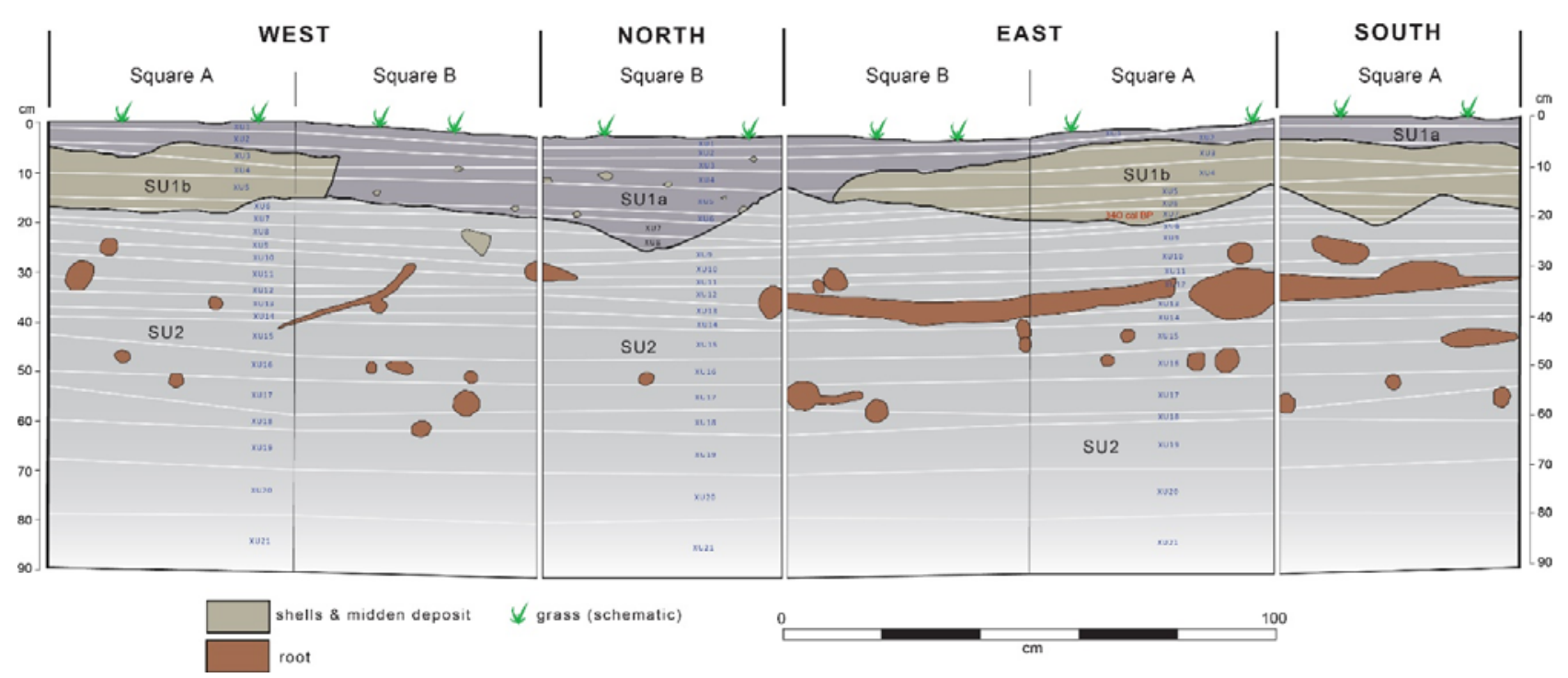

Figure 8. Stratigraphic sections for Squares A and B, Site 75b.

Table 1. Radiocarbon age determinations for Tin Can Inlet Sites 62 and $75 \mathrm{~b}$.

\begin{tabular}{|c|c|c|c|c|c|c|c|c|c|}
\hline Site & $\begin{array}{c}\text { Lab. } \\
\text { No. }\end{array}$ & $\begin{array}{c}\text { Depth } \\
\text { Below } \\
\text { Surface } \\
\text { (cm) }\end{array}$ & SU & SqXU & $\begin{array}{c}\text { Sample } \\
\text { Type }\end{array}$ & $\begin{array}{c}\text { Conventional } \\
{ }^{14} \text { C Age } \\
\text { (years BP) }\end{array}$ & $\begin{array}{c}\text { Calibrated Age BP } \\
68.3 \% \\
\text { (probabilities) }\end{array}$ & $\begin{array}{c}\text { Calibrated Age BP } \\
95.4 \% \\
\text { (probabilities) }\end{array}$ & $\begin{array}{c}\text { Median } \\
\text { Age (cal } \\
\text { BP) }\end{array}$ \\
\hline 62 & $\begin{array}{l}\text { Beta- } \\
34400\end{array}$ & $14-17$ & 1 & B6 & charcoal & $190 \pm 50$ & $\begin{array}{c}0-21(0.113) \\
71-93(0.107) \\
103-113(0.053) \\
139-157(0.107) \\
164-230(0.376) \\
241-281(0.243)\end{array}$ & $\begin{array}{c}0-47(0.141) \\
53-125(0.237) \\
131-291(0.622)\end{array}$ & 165 \\
\hline 62 & $\begin{array}{l}\text { Beta- } \\
34401\end{array}$ & $27-30$ & 2 & B10 & charcoal & $950 \pm 60$ & $\begin{array}{l}760-823(0.491) \\
827-844(0.114) \\
855-905(0.395)\end{array}$ & $\begin{array}{l}690-699(0.009) \\
721-930(0.991)\end{array}$ & 824 \\
\hline $75 b$ & $\begin{array}{l}\text { Beta- } \\
19421\end{array}$ & $18-21$ & $1 \mathrm{~b}$ & A7 & $\begin{array}{l}\text { marine } \\
\text { shell* }\end{array}$ & $700 \pm 70$ & $274-414(1.000)$ & $\begin{array}{l}147-164(0.014) \\
187-479(0.986)\end{array}$ & 340 \\
\hline
\end{tabular}

* Mixed sample of oyster (Saccostrea spp.) and Hercules club whelk (P. ebeninus). 
Table 2. Data recordings for Square A, Site 75b (after McNiven 1990a:403, Table A.25, 1991a:104).

\begin{tabular}{|c|c|c|c|c|c|c|c|c|c|}
\hline SU & $\mathbf{X U}$ & $\begin{array}{l}\text { Mean XU } \\
\text { Thickness } \\
\text { (cm) }\end{array}$ & $\begin{array}{c}\text { Mean XU } \\
\text { Depth } \\
\text { Below } \\
\text { Surface } \\
\text { (cm) } \\
\end{array}$ & $\begin{array}{c}\text { XU } \\
\text { Weight } \\
\text { (kg) }\end{array}$ & pH & $\begin{array}{c}\text { Charcoal } \\
\text { (g) }\end{array}$ & $\begin{array}{c}\text { Shell } \\
\text { (g) }\end{array}$ & $\begin{array}{c}\text { Stone } \\
\text { Artefacts } \\
\text { (g) }\end{array}$ & $\begin{array}{c}\text { Median } \\
\text { Age } \\
\text { (cal BP) }\end{array}$ \\
\hline 1 & 1 & 2 & 2 & 5.5 & 4.5 & 19.34 & 21.4 & 0.05 & \\
\hline 1 & 2 & 3 & 5 & 7.7 & - & 12.52 & 344.9 & 0.20 & \\
\hline 1 & 3 & 3 & 8 & 11.1 & 5.0 & 11.00 & 1679.9 & 0.33 & \\
\hline 1 & 4 & 4 & 12 & 12.7 & - & 31.11 & 2361.5 & 0.10 & \\
\hline 1 & 5 & 4 & 16 & 12.7 & 7.0 & 17.90 & 1568.5 & 131.36 & \\
\hline 1 & 6 & 2 & 18 & 9.1 & - & 8.17 & 491.6 & 0.89 & \\
\hline $1 / 2$ & 7 & 3 & 21 & 8.9 & 7.5 & 6.61 & 151.0 & 0.09 & 340 \\
\hline 2 & 8 & 2 & 23 & 8.6 & - & 3.77 & 28.9 & 0.14 & \\
\hline 2 & 9 & 5 & 28 & 10.5 & 7.0 & 4.25 & 3.9 & 4.05 & \\
\hline 2 & 10 & 3 & 31 & 9.6 & - & 3.40 & 1.2 & 1.10 & \\
\hline 2 & 11 & 3 & 34 & 9 & 8.0 & 1.59 & 0.4 & 0.34 & \\
\hline 2 & 12 & 3 & 37 & 10.5 & - & 1.20 & 0.3 & 0.07 & \\
\hline 2 & 13 & 3 & 40 & 9.9 & 7.5 & 2.15 & 0.6 & 0.19 & \\
\hline 2 & 14 & 2 & 42 & 7.9 & - & 0.79 & 0.1 & 0.08 & \\
\hline 2 & 15 & 5 & 47 & 16.7 & 7.0 & 0.50 & 0.8 & 0.19 & \\
\hline 2 & 16 & 5 & 52 & 19.2 & - & 0.29 & 0.1 & 0.19 & \\
\hline 2 & 17 & 6 & 58 & 20.6 & 7.5 & 0.39 & 0.8 & 0.19 & \\
\hline 2 & 18 & 4 & 62 & 18.6 & - & 0.14 & 0.1 & 0.05 & \\
\hline 2 & 19 & 9 & 71 & 32.2 & 7.0 & 0.03 & 0.1 & 0.33 & \\
\hline 2 & 20 & 10 & 81 & 37.5 & - & 0.06 & 0 & 0.41 & \\
\hline 2 & 21 & 11 & 92 & 41.2 & 6.5 & 0.08 & 0.1 & 0.68 & \\
\hline Total & & & & 319.7 & & 125.29 & 6656.2 & 141.03 & \\
\hline
\end{tabular}

Table 3. Mollusc MNI by XU for Square A, Site 75b (after McNiven 1990a:197, 1991a:93).

\begin{tabular}{|c|c|c|c|c|c|c|c|c|c|c|c|c|c|c|}
\hline Taxon & 1 & 2 & 3 & 4 & 5 & 6 & 7 & 8 & 9 & 11 & 12 & 17 & Total MNI & $\%$ MNI \\
\hline Anadara trapezia & 0 & 0 & 0 & 5 & 8 & 0 & 0 & 0 & 0 & 0 & 0 & 0 & 13 & 1.43 \\
\hline Bedeva paivae & 0 & 0 & 0 & 1 & 1 & 0 & 0 & 0 & 0 & 0 & 0 & 0 & 2 & 0.22 \\
\hline Bembicium nanum & 0 & 1 & 1 & 2 & 2 & 0 & 0 & 0 & 0 & 0 & 0 & 0 & 6 & 0.66 \\
\hline Cerithidea largillierti $^{1}$ & 0 & 0 & 2 & 6 & 6 & 0 & 0 & 0 & 0 & 0 & 0 & 0 & 14 & 1.54 \\
\hline Conuber sordidum ${ }^{2}$ & 0 & 0 & 0 & 3 & 27 & 5 & 1 & 0 & 0 & 0 & 0 & 0 & 36 & 3.96 \\
\hline Ergalatax contracta ${ }^{3}$ & 0 & 0 & 0 & 0 & 1 & 0 & 0 & 0 & 0 & 0 & 0 & 0 & 1 & 0.11 \\
\hline Isognomon sp. ${ }^{4}$ & 3 & 21 & 38 & 5 & 0 & 0 & 0 & 0 & 0 & 0 & 0 & 0 & 67 & 7.37 \\
\hline Mactridae & 0 & 0 & 1 & 0 & 0 & 0 & 0 & 0 & 0 & 0 & 0 & 0 & 1 & 0.11 \\
\hline Nerita balteata ${ }^{5}$ & 0 & 0 & 0 & 0 & 2 & 12 & 0 & 0 & 0 & 0 & 0 & 0 & 14 & 1.54 \\
\hline Patelloida mimula & 0 & 0 & 1 & 2 & 1 & 1 & 1 & 0 & 0 & 0 & 0 & 0 & 6 & 0.66 \\
\hline Plebidonax deltoides ${ }^{6}$ & 0 & 0 & 1 & 3 & 0 & 0 & 0 & 0 & 0 & 0 & 0 & 0 & 4 & 0.44 \\
\hline Pyrazus ebeninus & 0 & 0 & 16 & 40 & 35 & 29 & 12 & 1 & 1 & 1 & 1 & 1 & 137 & 15.07 \\
\hline Saccostrea spp. ${ }^{7}$ & 4 & 41 & 90 & 249 & 168 & 38 & 11 & 3 & 0 & 0 & 0 & 0 & 604 & 66.45 \\
\hline Trichomya hirsuta & 0 & 2 & 2 & 0 & 0 & 0 & 0 & 0 & 0 & 0 & 0 & 0 & 4 & 0.44 \\
\hline Total & 7 & 65 & 152 & 316 & 251 & 85 & 25 & 4 & 1 & 1 & 1 & 1 & 909 & 100 \\
\hline
\end{tabular}

${ }^{1}$ Velacumantus australis in original text, ${ }^{2}$ syn. Polinices sordidus, ${ }^{3}$ Formerly Cronia contracta, ${ }^{4}$ Ephippium ephippium in original text, ${ }^{5}$

Formerly Nerita lineata, ${ }^{6}$ Formerly Donax deltoides, ${ }^{7}$ Saccostrea commercialis in original text. 
Table 4. Data recordings for Square B, Site 75b (after McNiven 1990a:404).

\begin{tabular}{|c|c|c|c|c|c|c|c|c|}
\hline SU & $\mathbf{X U}$ & $\begin{array}{l}\text { Mean XU } \\
\text { Thickness } \\
\text { (cm) }\end{array}$ & $\begin{array}{c}\text { Mean XU } \\
\text { Depth Below } \\
\text { Ground Surface } \\
\text { (cm) }\end{array}$ & $\begin{array}{c}\text { XU } \\
\text { Weight } \\
\text { (kg) }\end{array}$ & $\begin{array}{c}\text { Charcoal } \\
(\mathrm{g})^{*}\end{array}$ & $\begin{array}{c}\text { Bone } \\
(\mathrm{g})^{*}\end{array}$ & $\begin{array}{l}\text { Shell } \\
\text { (g)* }\end{array}$ & $\begin{array}{c}\text { Stone } \\
\text { Artefacts } \\
\text { (g) }\end{array}$ \\
\hline 1 & 1 & 2 & 2 & 6.0 & 0 & 0 & 6.73 & 0 \\
\hline 1 & 2 & 2 & 4 & 7.5 & 0 & 0 & 48.79 & 0 \\
\hline 1 & 3 & 4 & 8 & 10.5 & 9.33 & 0.09 & 926.34 & 0 \\
\hline 1 & 4 & 2 & 10 & 10.3 & 13.70 & 0.24 & 579.95 & 1.64 \\
\hline 1 & 5 & 5 & 15 & 13.3 & 16.11 & 0 & 831.73 & 4.74 \\
\hline $1 / 2$ & 6 & 2 & 17 & 9.7 & 10.29 & 0.01 & 316.59 & 0.12 \\
\hline 2 & 7 & 3 & 20 & 9.6 & 6.33 & 0 & 69.15 & 0.04 \\
\hline 2 & 8 & 3 & 23 & 9.5 & 4.92 & 0 & 0 & 0.27 \\
\hline 2 & 9 & 2 & 25 & 8.8 & 5.45 & 0 & 0 & 1.02 \\
\hline 2 & 10 & 3 & 28 & 8.9 & 0 & 0 & 0 & 0.06 \\
\hline 2 & 11 & 3 & 31 & 9.3 & 0 & 0 & 0 & 0.12 \\
\hline 2 & 12 & 2 & 33 & 8.5 & 0 & 0 & 0 & 0 \\
\hline 2 & 13 & 4 & 37 & 9.5 & 0 & 0 & 0 & 0 \\
\hline 2 & 14 & 2 & 39 & 8.8 & 0 & 0 & 0 & 0.03 \\
\hline 2 & 15 & 6 & 45 & 19.7 & 0 & 0 & 0 & 0.26 \\
\hline 2 & 16 & 5 & 50 & 18.0 & 0 & 0 & 0 & 0.16 \\
\hline 2 & 17 & 6 & 56 & 17.4 & 0 & 0 & 0 & 0.03 \\
\hline 2 & 18 & 4 & 60 & 21.7 & 0 & 0 & 0 & 0.15 \\
\hline 2 & 19 & 9 & 69 & 28.4 & 0 & 0 & 0 & 0.11 \\
\hline 2 & 20 & 9 & 78 & 37.1 & 0 & 0 & 0 & 0.33 \\
\hline 2 & 21 & 11 & 89 & 38.1 & 0 & 0 & 0 & 1.01 \\
\hline Total & & & & 310.6 & 66.13 & 0.34 & 2779.28 & 10.09 \\
\hline
\end{tabular}

*Absent from the original data and recorded during Smith's (2016) study.

Table 5. Mollusc NISP by XU for Square B, Site 75b.

\begin{tabular}{|l|r|r|r|r|r|r|r|r|r|}
\hline \multicolumn{1}{|c|}{ Taxon } & \multicolumn{1}{c|}{$\mathbf{2}$} & \multicolumn{1}{c|}{$\mathbf{3}$} & \multicolumn{1}{c|}{$\mathbf{5}$} & \multicolumn{1}{c|}{$\mathbf{6}$} & $\mathbf{7}$ & Total NISP & \% NISP \\
\hline Anadara trapezia & 1 & 0 & 0 & 13 & 19 & 0 & 0 & 33 & 0.76 \\
\hline Bembicium sp. & 0 & 0 & 2 & 2 & 0 & 0 & 0 & 4 & 0.09 \\
\hline Cerithium spp. & 0 & 0 & 6 & 0 & 0 & 1 & 0 & 7 & 0.16 \\
\hline Conuber sordidum & 0 & 0 & 0 & 1 & 22 & 5 & 1 & 29 & 0.66 \\
\hline Fluviolanatus subtorta & 1 & 0 & 0 & 0 & 0 & 0 & 0 & 1 & 0.02 \\
\hline Isognomon spp. & 0 & 215 & 854 & 60 & 0 & 0 & 0 & 1129 & 25.84 \\
\hline Nerita balteata & 0 & 0 & 3 & 1 & 0 & 0 & 0 & 4 & 0.09 \\
\hline Plebidonax deltoides & 1 & 9 & 0 & 0 & 0 & 0 & 0 & 10 & 0.23 \\
\hline Pyrazus ebeninus & 0 & 0 & 20 & 60 & 196 & 101 & 33 & 410 & 9.38 \\
\hline Saccostrea spp. & 17 & 4 & 529 & 1297 & 557 & 204 & 91 & 2699 & 61.78 \\
\hline Trichomya hirsuta & 0 & 1 & 37 & 0 & 5 & 0 & 0 & 43 & 0.98 \\
\hline Total & $\mathbf{2 0}$ & $\mathbf{2 2 9}$ & $\mathbf{1 4 5 1}$ & $\mathbf{1 4 3 4}$ & $\mathbf{7 9 9}$ & $\mathbf{3 1 1}$ & $\mathbf{1 2 5}$ & $\mathbf{4 3 6 9}$ & $\mathbf{1 0 0}$ \\
\hline
\end{tabular}


Table 6. Mollusc MNI by XU for Square B, Site $75 b$.

\begin{tabular}{|l|r|r|r|r|r|r|r|r|r|}
\hline \multicolumn{1}{|c|}{ Taxon } & $\mathbf{1}$ & $\mathbf{2}$ & $\mathbf{3}$ & \multicolumn{1}{c|}{$\mathbf{4}$} & $\mathbf{5}$ & $\mathbf{6}$ & $\mathbf{7}$ & Total MNI & \% MNI \\
\hline Anadara trapezia & 1 & 0 & 0 & 3 & 7 & 0 & 0 & 11 & 3.16 \\
\hline Bembicium auratum & 0 & 0 & 2 & 2 & 0 & 0 & 0 & 4 & 1.15 \\
\hline Cerithium sp. & 0 & 0 & 6 & 0 & 0 & 1 & 0 & 7 & 2.01 \\
\hline Conuber sordidum & 0 & 0 & 0 & 1 & 10 & 4 & 1 & 16 & 4.60 \\
\hline Fluviolanatus subtorta & 1 & 0 & 0 & 0 & 0 & 0 & 0 & 1 & 0.29 \\
\hline Isognomon spp. & 0 & 4 & 29 & 2 & 0 & 0 & 0 & 35 & 10.06 \\
\hline Nerita balteata & 0 & 0 & 3 & 1 & 0 & 0 & 0 & 4 & 1.15 \\
\hline Plebidonax deltoides & 1 & 1 & 0 & 0 & 0 & 0 & 0 & 2 & 0.57 \\
\hline Pyrazus ebeninus & 0 & 0 & 8 & 15 & 32 & 38 & 9 & 102 & 29.31 \\
\hline Saccostrea spp. & 3 & 1 & 55 & 57 & 35 & 7 & 1 & 159 & 45.69 \\
\hline Trichomya hirsuta & 0 & 1 & 4 & 0 & 2 & 0 & 0 & 7 & 2.01 \\
\hline Total & $\mathbf{6}$ & $\mathbf{7}$ & $\mathbf{1 0 7}$ & $\mathbf{8 1}$ & $\mathbf{8 6}$ & $\mathbf{5 0}$ & $\mathbf{1 1}$ & $\mathbf{3 4 8}$ & $\mathbf{1 0 0}$ \\
\hline
\end{tabular}

Table 7. Volume-corrected mollusc MNI estimates for dominant species for Squares A and B, Site 75b.

\begin{tabular}{|c|c|c|c|c|c|c|c|c|c|}
\hline \multirow{2}{*}{\multicolumn{2}{|c|}{ Square A }} & SU1m ${ }^{3}$ & $\mathrm{SU} 2 \mathrm{~m}^{3}$ & Total & \multirow{2}{*}{\multicolumn{2}{|c|}{ Square B }} & SU1m ${ }^{3}$ & SU2 $\mathrm{m}^{3}$ & Total \\
\hline & & 0.053 & 0.178 & 0.23 & & & 0.053 & 0.178 & 0.23 \\
\hline \multirow[t]{2}{*}{ All shell } & MNI & 909 & 0 & 909 & \multirow[t]{2}{*}{ All shell } & MNI & 348 & 0 & 348 \\
\hline & $\mathrm{MNI} / \mathrm{m}^{3}$ & 17314 & 0 & 3952 & & $\mathrm{MNI} / \mathrm{m}^{3}$ & 0 & 0 & 1513 \\
\hline \multirow{2}{*}{$\begin{array}{l}\text { Anadara } \\
\text { trapezia }\end{array}$} & MNI & 13 & 0 & 13 & \multirow{2}{*}{$\begin{array}{l}\text { Anadara } \\
\text { trapezia }\end{array}$} & MNI & 11 & 0 & 11 \\
\hline & $\mathrm{MNI} / \mathrm{m}^{3}$ & 248 & 0 & 57 & & $\mathrm{MNI} / \mathrm{m}^{3}$ & 210 & 0 & 48 \\
\hline \multirow{2}{*}{$\begin{array}{l}\text { Conuber } \\
\text { sordidum }\end{array}$} & MNI & 36 & 0 & 36 & \multirow{2}{*}{$\begin{array}{l}\text { Conuber } \\
\text { sordidum }\end{array}$} & MNI & 16 & 0 & 16 \\
\hline & $\mathrm{MNI} / \mathrm{m}^{3}$ & 686 & 0 & 343 & & $\mathrm{MNI} / \mathrm{m}^{3}$ & 305 & 0 & 70 \\
\hline \multirow{2}{*}{$\begin{array}{l}\text { Isognomon } \\
\text { spp. }\end{array}$} & MNI & 67 & 0 & 67 & \multirow{2}{*}{$\begin{array}{l}\text { Isognomon } \\
\text { spp. }\end{array}$} & MNI & 35 & 0 & 35 \\
\hline & $\mathrm{MNI} / \mathrm{m}^{3}$ & 1276 & 0 & 291 & & $\mathrm{MNI} / \mathrm{m}^{3}$ & 667 & 0 & 152 \\
\hline \multirow{2}{*}{$\begin{array}{l}\text { Pyrazus } \\
\text { ebeninus }\end{array}$} & MNI & 132 & 5 & 137 & \multirow{2}{*}{$\begin{array}{l}\text { Pyrazus } \\
\text { ebeninus }\end{array}$} & MNI & 102 & 0 & 102 \\
\hline & $\mathrm{MNI} / \mathrm{m}^{3}$ & 2514 & 28 & 596 & & $\mathrm{MNI} / \mathrm{m}^{3}$ & 1943 & 0 & 443 \\
\hline \multirow{2}{*}{$\begin{array}{l}\text { Saccostrea } \\
\text { spp. }\end{array}$} & MNI & 601 & 3 & 604 & \multirow{2}{*}{$\begin{array}{l}\text { Saccostrea } \\
\text { spp. }\end{array}$} & MNI & 105 & 0 & 105 \\
\hline & $\mathrm{MNI} / \mathrm{m}^{3}$ & 11448 & 17 & 2626 & & $\mathrm{MNI} / \mathrm{m}^{3}$ & 2000 & 0 & 457 \\
\hline
\end{tabular}

Table 8. Mollusc weights (g) by XU for Square B, Site $75 \mathrm{~b}$.

\begin{tabular}{|l|r|r|r|r|r|r|r|r|r|}
\hline \multicolumn{1}{|c|}{ Taxon } & \multicolumn{1}{c|}{$\mathbf{1}$} & \multicolumn{1}{c|}{$\mathbf{2}$} & \multicolumn{1}{c|}{$\mathbf{4}$} & $\mathbf{5}$ & $\mathbf{6}$ & $\mathbf{7}$ & Total (g) & \% Weight \\
\hline Anadara trapezia & 0.43 & 14.11 & 72.7 & 0 & 0 & 0 & 0 & 87.24 & 3.14 \\
\hline Bembicium auratum & 0 & 0 & 0.55 & 0.19 & 0 & 0 & 0 & 0.74 & 0.03 \\
\hline Cerithium sp. & 0 & 0 & 13.01 & 0 & 0 & 2.62 & 0 & 15.63 & 0.56 \\
\hline Conuber sordidum & 0 & 0 & 0 & 1.50 & 35.07 & 0 & 0 & 36.57 & 1.32 \\
\hline Fluviolanatus subtorta & 0.09 & 0 & 0 & 0 & 0 & 0 & 0 & 0.09 & 0.00 \\
\hline Isognomon sp. & 0 & 26.86 & 427.66 & 43.84 & 0 & 0 & 0 & 498.36 & 17.93 \\
\hline Nerita balteata & 0 & 0 & 0.33 & 0.34 & 0 & 0 & 0 & 0.67 & 0.02 \\
\hline Plebidonax deltoides & 1.33 & 2.79 & 0 & 0 & 0 & 0 & 0 & 4.12 & 0.15 \\
\hline Pyrazus ebeninus & 0 & 0 & 26.06 & 106.23 & 473.68 & 229.78 & 47.29 & 883.04 & 31.77 \\
\hline Saccostrea spp. & 4.45 & 4.43 & 384.51 & 426.87 & 302.63 & 73.81 & 18.28 & 1214.98 & 43.72 \\
\hline Trichomya hirsuta & 0 & 0.60 & 1.52 & 0.07 & 0 & 0 & 0 & 2.19 & 0.08 \\
\hline Indeterminate & 0.43 & 0 & 0 & 0.91 & 20.35 & 10.38 & 3.58 & 35.65 & 1.28 \\
\hline Total & $\mathbf{6 . 7 3}$ & $\mathbf{4 8 . 7 9}$ & $\mathbf{9 2 6 . 3 4}$ & $\mathbf{5 7 9 . 9 5}$ & $\mathbf{8 3 1 . 7 3}$ & $\mathbf{3 1 3 . 9 7}$ & $\mathbf{6 9 . 1 5}$ & $\mathbf{2 7 7 9 . 2 8}$ & $\mathbf{1 0 0}$ \\
\hline
\end{tabular}


Table 9. Combined mollusc MNI for Squares A and B, Site 75b.

\begin{tabular}{|l|r|r|r|r|}
\hline \multicolumn{1}{|c|}{ Taxon } & Square A & Square B & Total MNI & \% MNI \\
\hline Anadara trapezia & 13 & 11 & 24 & 1.91 \\
\hline Bedeva paivae & 2 & 0 & 2 & 0.16 \\
\hline Bembicium spp. & 6 & 4 & 10 & 0.80 \\
\hline Cerithium sp. & 14 & 7 & 21 & 1.59 \\
\hline Conuber sordidum & 36 & 16 & 52 & 4.14 \\
\hline Ergalatax contracta & 1 & 0 & 1 & 0.08 \\
\hline Fluviolanatus subtorta & 0 & 1 & 1 & 0.08 \\
\hline Isognomon spp. & 67 & 35 & 102 & 8.12 \\
\hline Mactridae & 1 & 0 & 1 & 0.08 \\
\hline Nerita balteata & 14 & 4 & 18 & 1.43 \\
\hline Patelloida mimula & 6 & 0 & 6 & 0.48 \\
\hline Plebidonax deltoides & 4 & 2 & 6 & 0.48 \\
\hline Pyrazus ebeninus & 137 & 102 & 239 & 19.03 \\
\hline Saccostrea spp. & 604 & 159 & 763 & 60.75 \\
\hline Trichomya hirsuta & 4 & 7 & 11 & 0.88 \\
\hline Total & $\mathbf{9 0 9}$ & $\mathbf{3 4 8}$ & $\mathbf{1 2 5 7}$ & $\mathbf{1 0 0}$ \\
\hline
\end{tabular}

Table 10. Molluscan diversity measures for Squares A and B, Site $75 \mathrm{~b}$.

\begin{tabular}{|l|r|r|}
\hline \multicolumn{1}{|c|}{ Diversity Measure } & \multicolumn{1}{c|}{ Square A } & \multicolumn{1}{c|}{ Square B } \\
\hline NTAXA & 14 & 11 \\
\hline Individuals & 909 & 348 \\
\hline Simpson's Index (1-D) & 0.528 & 0.691 \\
\hline Shannon Index of Diversity ('H) & 1.209 & 1.506 \\
\hline Shannon Index of Evenness (E) & 0.458 & 0.6279 \\
\hline
\end{tabular}

Table 11. Descriptive statistics for Saccostrea spp. for Square B, SU1b, Site 75b.

\begin{tabular}{|c|c|r|r|r|r|r|}
\hline Attribute & \multicolumn{1}{|c|}{ N } & \multicolumn{1}{c|}{ Minimum } & \multicolumn{1}{c|}{ Maximum } & \multicolumn{1}{c|}{ Mean } & \multicolumn{1}{c|}{ Range } & \multicolumn{1}{c|}{ Std. Deviation } \\
\hline Valve height $(\mathrm{mm})$ & 72 & 14.96 & 65.36 & 36.0917 & 50.40 & 11.52514 \\
\hline Valve length $(\mathrm{mm})$ & 71 & 6.4 & 42.10 & 24.0065 & 35.7 & 7.47222 \\
\hline Valve depth $(\mathrm{mm})$ & 47 & 2.71 & 26.9 & 10.0589 & 24.9 & 4.83316 \\
\hline
\end{tabular}




\section{Taxon-Specific Analysis (Square B)}

\section{Saccostrea spp.}

There is some debate as to whether the oysters found in middens in southeast Queensland are either S. glomerata (Sydney rock oyster) and/or S. cucullata (hooded rock oyster) (e.g. Buroker et al. 1979; Healy and Potter 2010; Healy et al. 2011; Lam and Morton 2006; Thomson 1954). Oysters live attached to hard objects and are usually gregarious and often densely packed, leading to frequent morphological variability among individuals - what are described as separate species may in fact simply be ecophenotypical variations (Carpenter and Niem 1998:224). A conservative approach is taken here and the taxon of oyster present is referred to simply as Saccostrea spp.

Saccostrea spp. was the most abundant taxon in Square B, with a NISP of 2699 (Table 5), and an MNI of 159 (Table 6). The rank order did not change with volume-corrected estimates of MNI (Table 7). Heights were obtained for 72 valves, lengths for 71 valves, and depths for 47 valves (descriptive statistics are detailed in Table 11).

Single sample $t$ test results indicated statistically significant differences in both valve height $(t=26.572, d f=71$, $p<0.001)$ and valve length $(t=27.07, d f=70, p<0.001)$. However, further non-parametric Kolmogorov-Smirnov testing indicated a normal distribution for valve height and valve length $(D=0.044, \quad p>0.1$ and $D=0.038, \quad p>0.1$ respectively). Plotting the size frequency confirmed a generally normal distribution (Figure 9). Single sample $t$ test results indicated that there may be statistically significant differences in valve depth $(t=14.26, d f=46, p<0.001)$ and further non-parametric Kolmogorov-Smirnov tests confirmed this with a test statistic of $D=0.162$ and $p<0.05$. Plotting the depth frequencies indicated that the valve depths did not conform to a normal distribution curve (Figure 9).

Saccostrea spp. males reach sexual maturity at a height of 20mm (Catterall and Poiner 1987:120), therefore seven (9.7\%) of the 72 valves can be considered juveniles or subadults as they are between 14.96 and $19.56 \mathrm{~mm}$. Most of the valves from Square B fall into the $25-50 \mathrm{~mm}$ range with a peak in the $30-35 \mathrm{~mm}$ range (Figure 9) suggesting that the shellfish were harvested from a relatively mature population not subjected to over-exploitation.

Two valves exceeded $60 \mathrm{~mm}$ in height. Twenty-five of the 159 valves $(15.72 \%)$ included in the MNI exhibited the elongated hinges or beaks described by Galtsoff (1964:164) as indicative of 'old' oysters, reinforcing the contention that the population from which they were obtained included mature individuals. Only 19 of the valves (11.94\%) exhibited evidence of being cemented to other oysters, suggesting that the bed from which they were obtained was not tightly-packed or clustered. Eleven valves (6.91\%) were found cemented to two P. ebeninus shells (Figure 10), a phenomenon noted by Smith (1985), while a further 12 (7.5\%) had flat bases, implying that they grew directly either on rock or a firmly packed surface. Valve depth and morphology are environmentally influenced and Galtsoff (1964) noted that oysters attached to a pebble or shell, and so raised slightly from the bottom, had deep radially ribbed lower valves which offer greater resistance to dislodgement by currents or waves. The mean depth of the Saccostrea spp. valves is $10.06 \mathrm{~mm}$ and the frequency distribution is skewed towards depths between $2.71 \mathrm{~mm}$ and $12.5 \mathrm{~mm}$. This skewing implies that most of the oysters were not raised from the bottom sufficiently enough for them to develop tide-resistant characteristics. Unfortunately, the depths of the valves attached to P. ebeninus shells could not be measured.

The fragmentation ratio for Saccostrea spp. (NISP:MNI) was reasonably high at 16.97 . A moderately robust species (see Robins and Stock 1990), it is likely that the small numbers of Saccostrea spp. in the lowest levels were trampled at the commencement of midden deposition. This effect would have been exaggerated by the location of the shells within thinner deposits on the edge of the midden. None of the shells exhibited evidence of burning, implying that they were opened using low temperature fires, and therefore the integrity of the shell matrix was not weakened as a result.

\section{Pyrazus ebeninus}

P. ebeninus was the second most abundant species in Square $B$ in terms of MNI $(n=102)$, but ranked third in NISP $(n=410)$ (Table 6). The rank order did not change with volumecorrected estimates of MNI (Table 7). The MNI was derived from a count of whole specimens $(n=6)$, and apertures and apertural lips from broken or partial shells. All broken and partial shells demonstrated breakage patterns consistent with cultural processing for the removal of the snail (i.e. breaking off the top of the shell (apex) to break the suction) (see Meehan 1982). The breakages observed are unlikely to be the result of natural processes given the robustness of adult Hercules club whelk shells and the likelihood that humans are their only successful predators (John Healy, Queensland Museum, pers. comm., 2012). Additionally, the breakage pattern was consistent, with the majority of shells $(62.85 \%)$ broken at the level of the second or third whorl. As only six complete specimens were recovered, meaningful statistical analysis of shell height was not possible. However, it was noted that these specimens had an average height of $62.55 \mathrm{~mm}$, within the range $(50-70 \mathrm{~mm})$ at which sexual maturity is reached (Vohra 1965). As to be expected from such robust shells, the fragmentation ratio was low at 4.01. None of the shells exhibited evidence of burning, implying that they were cooked on low temperature fires.

\section{Isognomon spp.}

Isognomon spp. (commonly known as toothed pearl oyster, mangrove oyster, or tree oyster) was the second most abundant species in terms of NISP $(n=1129)$, but ranked third in the MNI $(n=35)$ (Tables 5-6). The rank order did not change with volume-corrected estimates of MNI (Table 7). Although the common names for the taxon imply association with mangroves (Carpenter and Niem 1998), they also attach to rocks or other shells by their byssal threads (Healy et al. 2011:171). Only nine complete valves were recovered, rendering meaningful statistical analyses of shell height and/or shell length impossible. I. ephippium, the species recorded by McNiven (1990a, 1991a), can reach a length (the maximum dimension across the valve, measured parallel to the hinge) of $140 \mathrm{~mm}$ but more commonly $100 \mathrm{~mm}$ (Carpenter and Niem 1998:191). Length measurements obtained from the nine complete valves ranged from $20 \mathrm{~mm}$ to $64.96 \mathrm{~mm}$, with a mean value of $44.26 \mathrm{~mm}$ (std. dev. 5.9). No literature is available on the overall growth rates of Isognomon spp., but it would appear from the potential adult size that the valves from Square B are juveniles or subadults. None of the shells 
exhibited evidence of burning. Isognomon spp. shells are thin, fragile, and subject to extensive post-mortem delamination. It is therefore unsurprising that the fragmentation ratio was high, and at 32.25 almost twice that recorded for Saccostrea.

\section{Conuber sordidum}

C. sordidum is the fourth most abundant species in both Square A and Square B (Table 9). Although it does not usually appear on the 'economic species' lists from southeast Queensland middens (although see Hall 1980, 1984, and Hall and Bowen 1989 for examples from Moreton Island), it is nevertheless a useful environmental indicator. Commonly known as the leaden or dirty sand snail, it is a carnivorous gastropod which buries itself in sandy sediments in order to ambush its prey, including soldier crabs, bivalves, and other species of sand snail (Healy et al. 2011:133). It typically grows to a maximum height of $40-50 \mathrm{~mm}$. Height measurements were obtained for 15 individuals from Square $\mathrm{B}$ and ranged from $6.61 \mathrm{~mm}$ to $36.1 \mathrm{~mm}$, with a mean value of $22.34 \mathrm{~mm}$, suggesting that most of the individuals were subadult. The shell is robust, with a fragmentation ratio for Square B of 1.8. None of the shells exhibited evidence of burning or exposure to high temperatures. Given the combined total of $C$. sordidum from both squares $(n=52)$, and the snails' habits, it seems an unlikely candidate for casual collection or by-catch, however no other explanation is immediately obvious (see Meehan 1982 for comments on collection methods for partially buried molluscs). Carnivorous or scavenging gastropods are known to clump together in groups (Fisheries Research Consultants 1994:26) and it may be that the C. sordidum shells reflect an opportunistic exploitation of an aggregated resource.

\section{Anadara trapezia}

The low number of $A$. trapezia (NISP=33, MNI=11, volumecorrected MNI estimate $=48$ ) is somewhat surprising given that the species frequently occurs in high numbers in southeast Queensland middens (e.g. Hope Island - Walters et al. 1987; Bribie Island - Smith 1992, 2003), and that it also occurs in habitats similar to those occupied by $P$. ebeninus. Length measurements were obtained from 10 valves and ranged from $30.13 \mathrm{~mm}$ to $51.47 \mathrm{~mm}$, with a mean value of $35.72 \mathrm{~mm}$ (std. dev. 5.9). This length range indicates that the individuals were sexually mature adults (Smith 2016). The valves are robust; the fragmentation ratio for Square B was 3. None of the shells exhibited evidence of burning.

\section{Trichomya hirsuta}

The numbers of $T$. hirsuta from Site $75 \mathrm{~b}$ are surprisingly low given that the species commonly occurs in estuarine middens in southeast Queensland (e.g. St Helena - Alfredson 1983; Booral Shell Mound - Frankland 1990). The MNI of seven was derived from nine valves (including two paired specimens) with a shell height range of $8.18-15.32 \mathrm{~mm}$, and a mean value of $10.49 \mathrm{~mm}$. Except for one pair of valves, all are considered juveniles, suggestive of by-catch and/or evidence of a recently established population (Smith 2016). The fragmentation ratio was 6.1 , which is low considering the thin walled nature of the valves. However juvenile mussels appear more resistant to trampling and breakage than larger specimens (Smith 2016). None of the shells exhibited evidence of burning.

\section{Plebidonax deltoides}

$P$. deltoides (pipi) is a common species in surf coast middens of southeast Queensland, dominating deposits after $1000 \mathrm{cal}$ BP (McNiven 2006). It occurs in small numbers in estuarine middens that have surf coast access but never in abundance to suggest dietary significance. $P$. deltoides at Site $75 \mathrm{~b}$ could have been obtained from Rainbow Beach and/or Teewah Beach, most likely from the former due to the shorter distance. The combined MNI for Site $75 \mathrm{~b}$ was six; a length of $33.6 \mathrm{~mm}$ from an incomplete valve from Square B indicates that the individual had probably reached sexual maturity (MurrayJones 1999). The regularly occurring low abundance suggests that pipis were introduced to the sites for non-dietary purposes (e.g. as raw materials for tool manufacture - see Smith 2016).

\section{Other Molluscan Species}

The remaining molluscan species most probably represent bycatch or collection for non-dietary purposes. All are useful environmental indicators. McNiven (1990a, 1991a) listed Cerithidea largillierti (mud creeper, $\mathrm{MNI}=14$ ) among the Square A taxa; Cerithium sp. (creeper, NISP=7, MNI=7) was identified in Square B. Although belonging to different families (Potamididae and Cerithiidae respectively), they are morphologically very similar (Wilson 1993) with some differentiating characteristics that do not survive archaeologically (apertural morphology, varix, rib morphology on the whorls). Both are detrital grazers on sandy-muddy estuarine sediments and similar species in both families grow to heights of $40-50 \mathrm{~mm}$. The seven ceriths from Square B ranged in height from $26.32 \mathrm{~mm}$ to $36.04 \mathrm{~mm}$, with a mean value of $31.09 \mathrm{~mm}$, suggesting that the individuals were subadult (Smith 2016). The sizes combined with the very low abundance adds support to the by-catch hypothesis. None of the shells exhibited evidence of breakage to extract the snail.

Nerita balteata (NISP $=4, \mathrm{MNI}=4)$ is an algal grazer that lives high in the intertidal zone on the trunks and branches of mangroves, and grows to a height of 40mm (Smith 2016). All specimens recovered from Square B were broken and individual heights could not be obtained. The Patelloida mimula recorded in Square A is a limpet which inhabits sheltered estuaries and bays, usually associated with Saccostrea spp. (Wilson 1993:34) and most likely represents by-catch. Bedeva paivae and Ergalatax contracta are both carnivorous gastropods, which prey on Saccostrea spp. and most likely represent oyster by-catch. Bembicium spp. (periwinkles) are grazing gastropods, with each species preferring slightly different habitats. Bembicium nanum was reported for Square A, however the species prefers moderately exposed rocky shores (Wilson 1993) at odds with the Tin Can Inlet environment. It is probable that the species was misidentified. Bembicium auratum was identified from Square B and prefers less exposed environments, is found on mangroves, and often in estuaries (Wilson 1993). The heights of two shells from Square B were $7.25 \mathrm{~mm}$ and $8.39 \mathrm{~mm}$, indicating that they were subadults. Fluviolanatus subtorta is a bivalve found in brackish water and coastal lagoons (Lamprell and Healy 1998:250) while Mactridae (trough clams) are deep-burrowing bivalves preferring sand or sandymud sediments (Healy et al. 2011:180). 

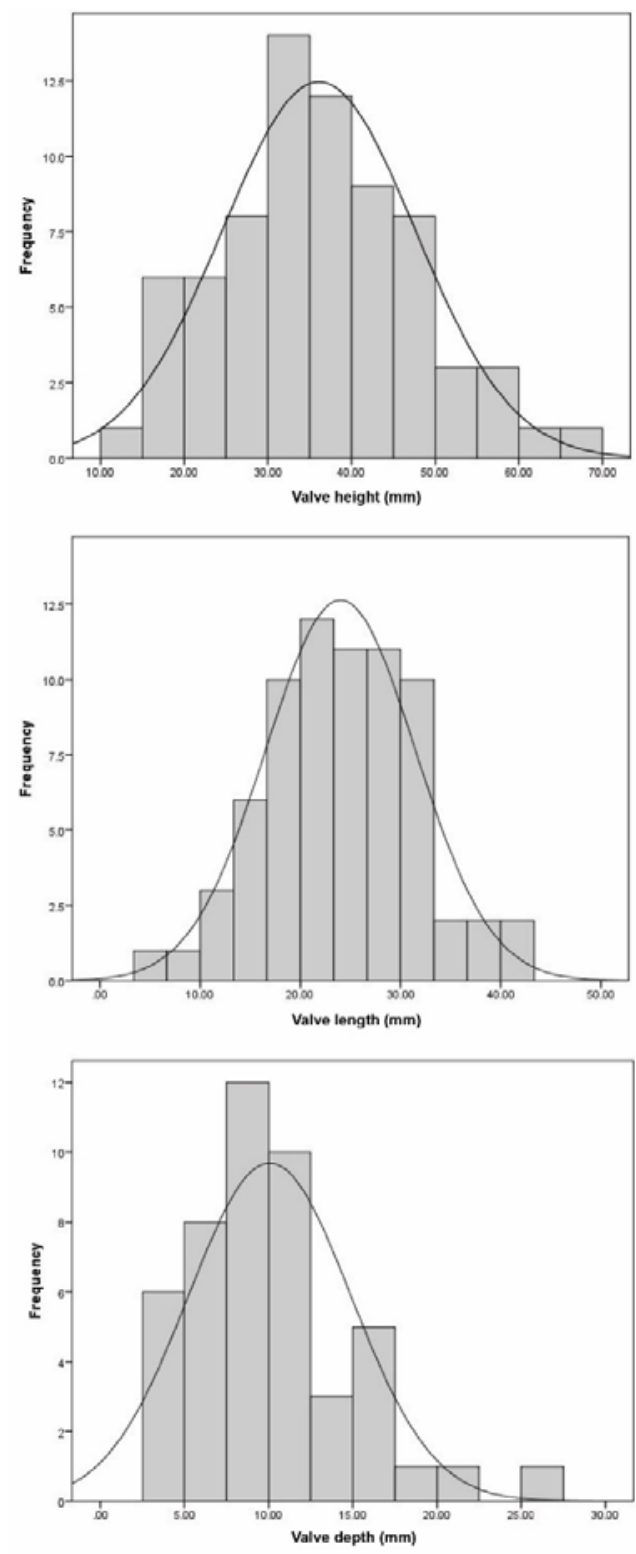

Figure 9. Histograms of Saccostrea spp. size frequency plotted against normal distribution curve for valve height, length and depth, Square B, Site 75b.

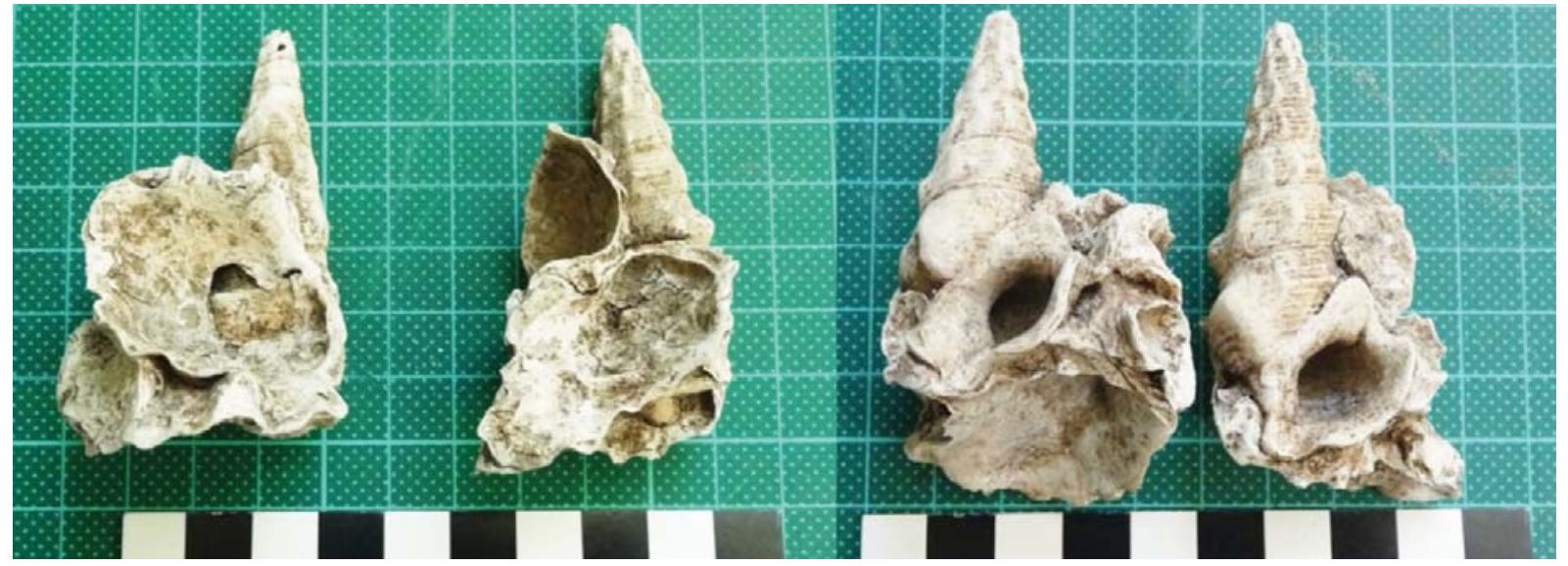

Figure 10. Oysters attached to Hercules club whelk shells from Site 75b Square B. Left: dorsal view.Right: ventral view. Scale in 1cm units (Photograph: Tam Smith). 


\section{Crustacean Remains (Square B)}

No crustacean remains were identified from Square A. In contrast, 12 crustacean fragments with a combined weight of $1.05 \mathrm{~g}$ was recovered from XUs 3-6 of Square B. The level of fragmentation prevented identification beyond the family level of Portunidae, but mud crabs (S. serrata) and sand crabs (P. pelagicus) occur within Tin Can Inlet, and are known to occur in Aboriginal midden deposits in southeast Queensland (e.g. Alfredson 1983; Smith 2016).

\section{Vertebrate Remains (Square B)}

Two fragments of fish bone weighing $0.01 \mathrm{~g}$ and a worn Sillago spp. otolith weighing $0.08 \mathrm{~g}$ were recovered. Terrestrial vertebrates comprised 16 fragments of unburnt bone $(0.09 \mathrm{~g})$, two burned bone fragments $(0.23 \mathrm{~g})$, and four burned fragments of long bone $(0.01 \mathrm{~g})$. The level of fragmentation prevented taxonomic identification of the burned bone, but the size and morphology suggest the bones belong to an animal the size of a small rodent. McNiven (1991a:93) noted two fragments of unburnt bone in XU1 of Square A, but was unconvinced that they represented discard by Aboriginal people, as he had observed bone fragments in recent dingo scats near the site and considered that the bone could equally represent weathered dingo faecal material (see McNiven 1990b for a detailed discussion of the taphonomy of faunal remains from blowouts along the Cooloola Coast, including the consumption of fish and shellfish by dingoes). The fish bone from Square B was similarly unburnt; the Sillago sp. otolith was worn whereas the associated molluscan remains were not. There was no clear evidence on the otolith of either mastication or gastric etching to support the possibility that it represented weathered dingo faecal material, however the worn nature of the element suggests secondary rather than primary deposition in the midden.

\section{Stone Artefacts (Square B)}

Eleven stone artefacts comprising three whole flakes, one retouched flake, four broken flakes and three flaked pieces were analysed in this study. Ten of the artefacts were of silcrete, and one of andesite. A laminar ferruginous sandstone manuport was also recorded. The artefacts were unremarkable (for details see Smith 2016) other than the retouched flake, which morphologically was a backed artefact.

\section{Tin Can Inlet Midden Site 62}

Site 62 is situated on the northern side of Cameron Point some $2 \mathrm{~km}$ north of Site $75 \mathrm{~b}$ (Figures 2, 4, 11) (for details see McNiven 1990a, 1991a; note that this site is also referred to as Cameron Point Site 62). The site was exposed for approximately $40 \mathrm{~m}$ along the upper sections of a $1.9 \mathrm{~m}$-high erosion face at the foot of which were lag deposits of stone artefacts and midden debris spread across the upper limits of the intertidal zone. As with Site 75b, the overall site area could not be estimated because of poor ground surface visibility but naturally eroded sections indicated that it was more than $5 \mathrm{~m}$ wide. The surrounding vegetation differed from that at Site $75 \mathrm{~b}$, being composed of forest red gum and coastal cypress forest. Three contiguous $50 \mathrm{~cm}$ x $50 \mathrm{~cm}$ pits (Squares A, B, C) were excavated in an area of relatively dense surface midden deposits between 21 and 22 November 1985. Squares B and $\mathrm{C}$ were each excavated to a depth of $52 \mathrm{~cm}$ using $13 \mathrm{XUs}$ with the excavated material dry-sieved through $3 \mathrm{~mm}$ mesh. The excavated volume of each square was $0.125 \mathrm{~m}^{3}$. In the original study, Square B was analysed in full, with stone artefacts from Square C also sorted and classified. No analysis was undertaken for Square A due to disturbance by tree roots.

Two major stratigraphic units were identified (Figures 1213). SU1 had a maximum depth of $22 \mathrm{~cm}$ and consisted of loose dark grey sand with a variable $\mathrm{pH}$ of 4.0-5.5. SU1 contained the bulk of the molluscs. SU2 extended from $13 \mathrm{~cm}$ below the surface to the base of the pit. Sediments graded from grey sand with a $\mathrm{pH}$ of 6.0 to light grey sand with a $\mathrm{pH}$ of 4.0. SU2 contained most of the stone artefacts (Table 12).

McNiven (1990a:203, 1991a:98) reported two radiocarbon ages on charcoal from Square $B$ which have been recalibrated using CALIB 7.10 and the Southern Hemisphere calibration dataset (SHCal13) (Hogg et al. 2013) (Table 1). The first from XU6 at a depth of $14-17 \mathrm{~cm}$ at the base of SU1 and the main midden deposit returned a conventional age of $190 \pm 50$ BP (Beta-34400) which produced a calibrated date of 0-291 cal BP (95.4\% probability) with a median age of 165 cal BP. The second from XU10 at a depth of $27-30 \mathrm{~cm}$ in SU2 returned a conventional age of $950 \pm 60$ BP (Beta-34401) which calibrated to $690-930 \mathrm{cal}$ BP ( $95.4 \%$ probability) with a median age of $824 \mathrm{cal} \mathrm{BP}$. Basal artefacts at the site, located $\sim 20 \mathrm{~cm}$ below the date in XU10, probably date to $<1000-1500$ years ago.

\section{Molluscs}

A total of $2854.5 \mathrm{~g}$ of shellfish comprising an MNI of 332 $\left(2656 / \mathrm{m}^{3}\right)$ from six taxa was recovered from Square B. As with Site $75 \mathrm{~b}$, the assemblage was dominated by Saccostrea spp.; the next most abundant species were $A$. trapezia and $P$. ebeninus, followed by three $T$. hirsuta and one each of $C$. largillierti and N. balteata (Table 13). Shell weights were recorded to the level of XU11 (Table 12), although no MNI was recorded below XU7 because of preservation conditions and a lack of diagnostic features for MNI calculation. No pipi ( $P$. deltoides) was excavated, but one valve was noted on the erosional face at the southern end of the site. McNiven (1991a:98) considered all species could be obtained from the intertidal zone adjacent to the site, while the pipi most likely came from Rainbow Beach some 6km away, although Teewah Beach, $13 \mathrm{~km}$ distant (Figure 1), could not be discounted.

Smith (2016) analysed the previously unsorted (other than the stone artefact component) contents of Square C (Table 14). There was a difference in the composition of the shellfish assemblage, in that mussels, ceriths, and nerites were entirely absent, and there was an occurrence of C. sordidum. No shells were recovered from the top two XUs, and the species diversity was low for an estuarine midden. This aspect is discussed further below. The NISP was 1025 (Table 15), and the total MNI was 182 (Table 16), with an estimated density of $1456 / \mathrm{m}^{3}$ (Table 17). Reflecting the fewer MNI, the shell weight (Table 18) is lower than that recorded for Square B. As for Site $75 b$, the shellfish data from Square C is initially presented here by $\mathrm{XU}$ to provide a comparison with those previously published, while the subsequent discussion is framed within the context of SU1 (the main midden deposit). Measures of richness, evenness, and diversity reflect low to moderate species diversity and richness, and the dominance of Saccostrea spp. in the assemblages, albeit less marked for Square C (Table 19). 


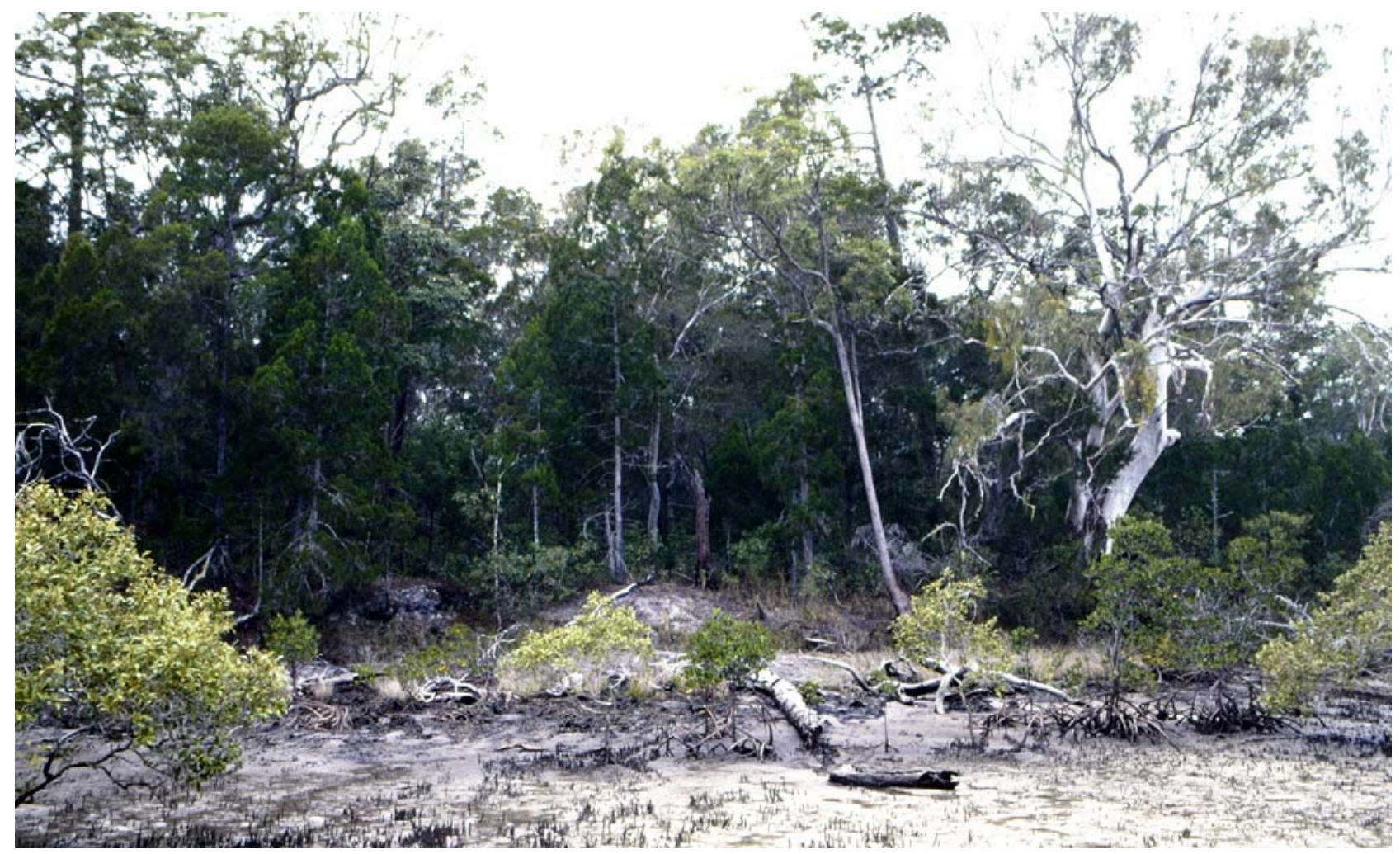

Figure 11. Site 62 (Photograph: Ian J. McNiven, November 1985).

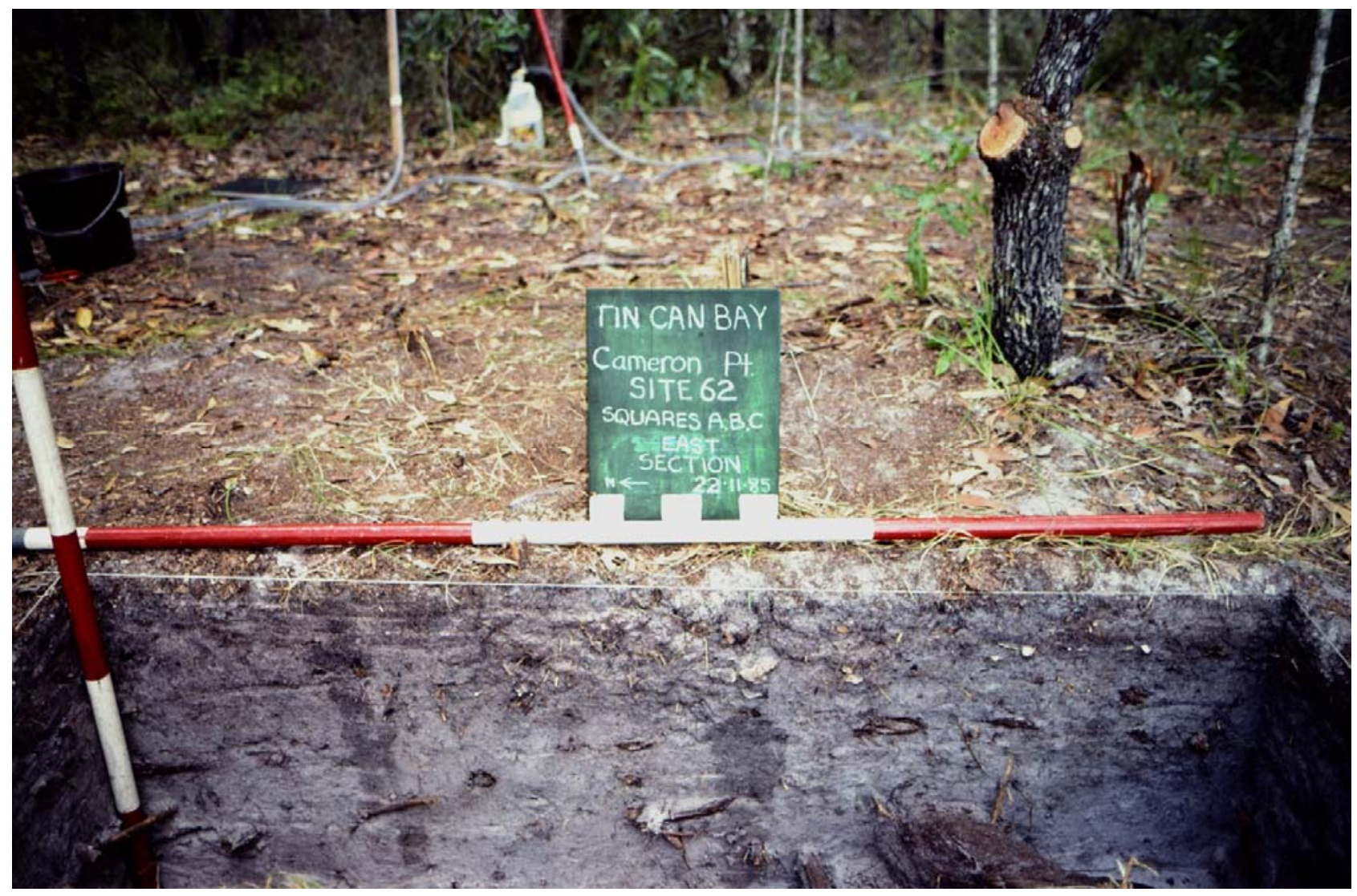

Figure 12. East section of Site 62 after excavation (Photograph: Ian J. McNiven, November 1985). 

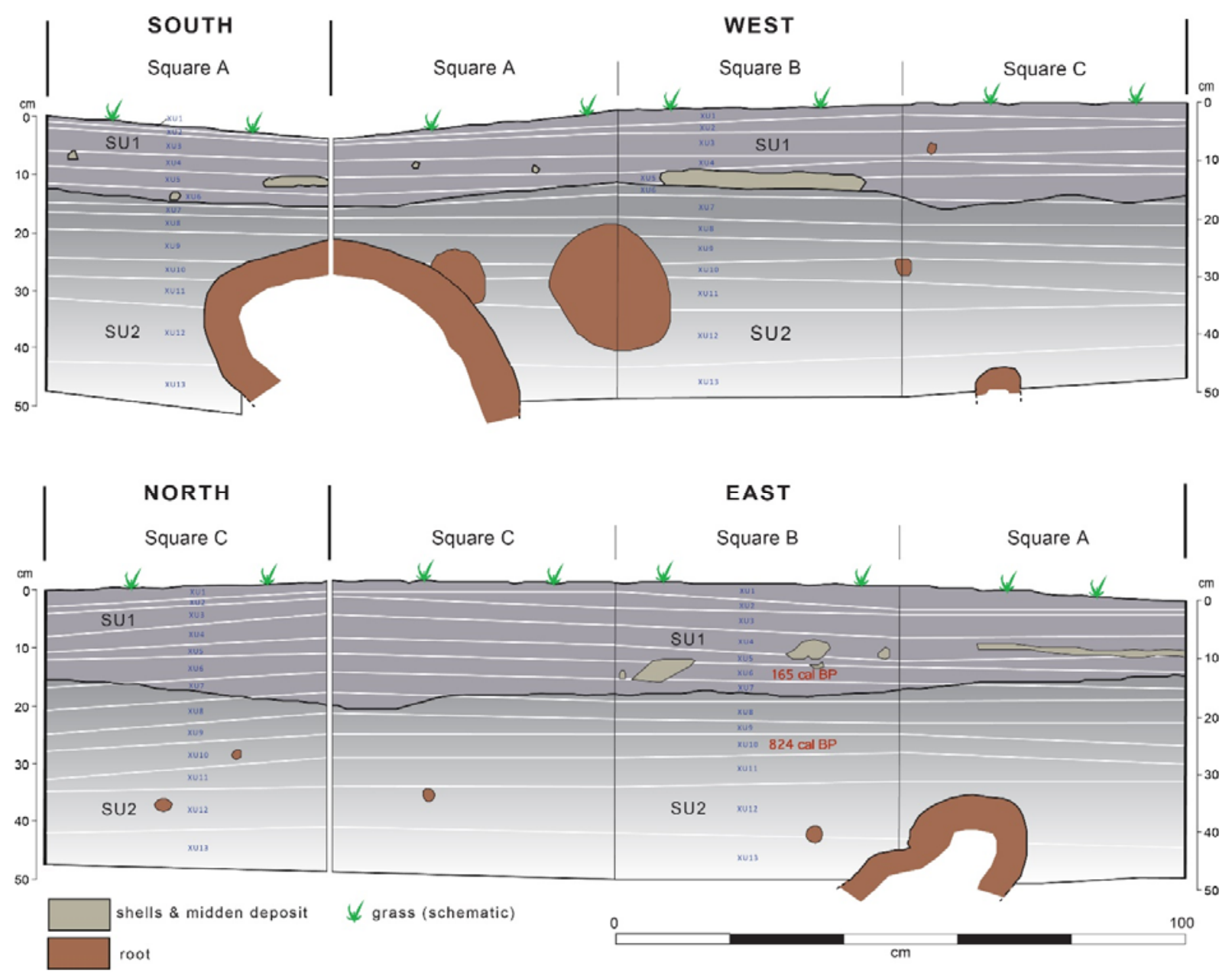

Figure 13. Stratigraphic sections for Squares A, B and C, Site 62. 
Table 12. Data recordings for Square B, Site 62 (after McNiven 1990a:404).

\begin{tabular}{|c|c|c|c|c|c|c|c|c|c|c|}
\hline SU & $\mathbf{X U}$ & $\begin{array}{c}\text { Mean XU } \\
\text { Thickness } \\
\quad(\mathrm{cm})\end{array}$ & $\begin{array}{c}\text { Mean XU } \\
\text { Depth Below } \\
\text { Surface } \\
\text { (cm) }\end{array}$ & $\begin{array}{l}\mathrm{XU} \\
(\mathrm{kg})\end{array}$ & pH & $\begin{array}{c}\text { Charcoal } \\
\text { (g) }\end{array}$ & $\begin{array}{l}\text { Shell } \\
\text { (g) }\end{array}$ & $\begin{array}{c}\text { Bone } \\
\text { (g) }\end{array}$ & $\begin{array}{c}\text { Stone } \\
\text { Artefacts } \\
\text { (g) }\end{array}$ & $\begin{array}{c}\text { Median } \\
\text { Age (cal } \\
\text { BP) }\end{array}$ \\
\hline 1 & 1 & 3 & 3 & 4.5 & 5 & 10.02 & 47.1 & 0 & 0 & \\
\hline 1 & 2 & 2 & 5 & 5.5 & & 15.40 & 4.00 & 0.10 & 0 & \\
\hline 1 & 3 & 4 & 9 & 6.1 & 4 & 9.64 & 111.5 & 0 & 0 & \\
\hline 1 & 4 & 3 & 12 & 8.0 & & 6.41 & 1689.6 & 0.01 & 0 & \\
\hline 1 & 5 & 2 & 14 & 7.3 & 5.5 & 33.79 & 884.6 & 0.04 & 0.24 & \\
\hline 1 & 6 & 3 & 17 & 7.6 & & 35.21 & 109.1 & 0 & 6.84 & 165 \\
\hline $1 / 2$ & 7 & 4 & 21 & 9.0 & 6 & 4.98 & 6.8 & 0 & 8.32 & \\
\hline 2 & 8 & 3 & 24 & 9.5 & & 7.65 & 1.3 & 0 & 1.05 & \\
\hline 2 & 9 & 3 & 27 & 10.1 & 5 & 14.68 & 0.1 & 0 & 0.20 & \\
\hline 2 & 10 & 3 & 30 & 10.5 & & 15.42 & 0.3 & 0 & 213.06 & 824 \\
\hline 2 & 11 & 5 & 35 & 11.3 & 5 & 12.78 & 0.1 & 0 & 2.29 & \\
\hline 2 & 12 & 8 & 43 & 20.7 & & 5.80 & 0 & 0 & 0.29 & \\
\hline 2 & 13 & 7 & 50 & 17.7 & 4 & 1.24 & 0 & 0 & 0.71 & \\
\hline Total & & & & 127.8 & & 173.02 & 2854.5 & 0.15 & 233 & \\
\hline
\end{tabular}

Table 13. Mollusc MNI by XU for Square B, Site 62 (after McNiven 1990a:205, 1991a:98).

\begin{tabular}{|l|r|r|r|r|r|r|r|r|r|}
\hline \multicolumn{1}{|c|}{ Taxon } & \multicolumn{1}{c|}{$\mathbf{1}$} & \multicolumn{1}{c|}{$\mathbf{2}$} & \multicolumn{1}{c|}{$\mathbf{1}$} & \multicolumn{1}{c|}{$\mathbf{4}$} & \multicolumn{1}{c|}{$\mathbf{6}$} & \multicolumn{1}{c|}{ T } & Total MNI & \% MNI \\
\hline Anadara trapezia & 0 & 0 & 1 & 4 & 10 & 2 & 0 & 17 & 5.12 \\
\hline Cerithidea largillierti & 0 & 0 & 0 & 0 & 1 & 0 & 0 & 1 & 0.30 \\
\hline Nerita balteata & 0 & 0 & 0 & 0 & 1 & 0 & 0 & 1 & 0.30 \\
\hline Pyrazus ebeninus & 4 & 0 & 2 & 18 & 9 & 3 & 1 & 37 & 11.14 \\
\hline Saccostrea spp. & 1 & 2 & 8 & 151 & 89 & 20 & 2 & 273 & 82.23 \\
\hline Trichomya hirsuta & 0 & 0 & 0 & 0 & 3 & 0 & 0 & 3 & 0.90 \\
\hline Total & $\mathbf{5}$ & $\mathbf{2}$ & $\mathbf{1 1}$ & $\mathbf{1 7 3}$ & $\mathbf{1 1 3}$ & $\mathbf{2 5}$ & $\mathbf{3}$ & $\mathbf{3 3 2}$ & $\mathbf{1 0 0 . 0 0}$ \\
\hline
\end{tabular}

Table 14. Data recordings for Square C, Site 62 (after McNiven 1990a:405, with additional data from Smith 2016 ).

\begin{tabular}{|c|c|c|c|c|c|c|c|c|}
\hline SU & $\mathbf{X U}$ & $\begin{array}{c}\text { Mean XU } \\
\text { Thickness } \\
\quad(\mathrm{cm}) \\
\end{array}$ & $\begin{array}{c}\text { Mean XU Depth } \\
\text { Below Surface } \\
(\mathrm{cm})\end{array}$ & $\begin{array}{l}\mathrm{XU} \\
(\mathrm{kg})\end{array}$ & $\begin{array}{c}\text { Charcoal } \\
\text { (g) }\end{array}$ & $\begin{array}{l}\text { Shell } \\
\text { (g) }\end{array}$ & $\begin{array}{c}\text { Bone } \\
\text { (g) }\end{array}$ & $\begin{array}{l}\text { Artefact } \\
\text { (g) }\end{array}$ \\
\hline 1 & 1 & 2 & 2 & 2.2 & 9.89 & 0 & 0 & $\underline{0}$ \\
\hline 1 & 2 & 2 & 4 & 4.6 & 14.88 & 0 & 1.35 & 0 \\
\hline 1 & 3 & 4 & 8 & 6.7 & 9.27 & 35.02 & 0.52 & 0 \\
\hline 1 & 4 & 2 & 10 & 6.3 & 6.38 & 429.19 & .0 .01 & 0 \\
\hline 1 & 5 & 3 & 13 & 6.6 & 34.01 & 807.96 & 0.04 & 0 \\
\hline 1 & 6 & 4 & 17 & 8.4 & 24.31 & 46.11 & 0 & 0 \\
\hline $1 / 2$ & 7 & 3 & 20 & 9.8 & 4.85 & 0 & 0 & 0 \\
\hline 2 & 8 & 4 & 24 & 9 & 7.3 & 0 & 0 & 0.50 \\
\hline 2 & 9 & 3 & 27 & 10.1 & 14.25 & 0 & 0 & 0.18 \\
\hline 2 & 10 & 4 & 31 & 10.5 & 3.3 & 0 & 0 & 213.36 \\
\hline 2 & 11 & 5 & 36 & 11.5 & 12.31 & 0 & 0 & 2.32 \\
\hline 2 & 12 & 7 & 43 & 20.7 & 5.44 & 0 & 0 & 0.23 \\
\hline 2 & 13 & 7 & 50 & 18.2 & 1.14 & 0 & 0 & 0 \\
\hline Total & & & & 124.6 & 147.33 & 1318.28 & 1.92 & 216.59 \\
\hline
\end{tabular}


Table 15. Mollusc NISP by XU for Square C, Site 62.

\begin{tabular}{|l|r|r|r|r|r|r|}
\hline \multicolumn{1}{|c|}{ Species } & $\mathbf{3}$ & $\mathbf{4}$ & $\mathbf{5}$ & $\mathbf{6}$ & Total NISP & \% NISP \\
\hline Anadara trapezia & 0 & 5 & 16 & 7 & 28 & 2.73 \\
\hline Conuber sordidum & 0 & 0 & 2 & 0 & 2 & 0.20 \\
\hline Pyrazus ebeninus & 1 & 8 & 48 & 21 & 78 & 7.61 \\
\hline Saccostrea spp. & 2 & 329 & 494 & 92 & 917 & 89.46 \\
\hline Total & $\mathbf{3}$ & $\mathbf{3 4 2}$ & $\mathbf{5 6 0}$ & $\mathbf{1 2 0}$ & $\mathbf{1 0 2 5}$ & $\mathbf{1 0 0 . 0 0}$ \\
\hline
\end{tabular}

Table 16. Mollusc MNI by XU for Square C, Site 62.

\begin{tabular}{|l|r|r|r|r|r|r|}
\hline \multicolumn{1}{|c|}{ Species } & \multicolumn{1}{c|}{$\mathbf{3}$} & \multicolumn{1}{c|}{$\mathbf{4}$} & \multicolumn{1}{c|}{$\mathbf{1}$} & \multicolumn{1}{c|}{ Total MNI } & \% MNI \\
\hline Anadara trapezia & 0 & 3 & 5 & 2 & 10 & 5.49 \\
\hline Conuber sordidum & 0 & 0 & 2 & 0 & 2 & 1.10 \\
\hline Pyrazus ebeninus & 1 & 6 & 18 & 3 & 28 & 15.38 \\
\hline Saccostrea spp. & 2 & 89 & 47 & 4 & 142 & 78.02 \\
\hline Total & $\mathbf{3}$ & $\mathbf{9 8}$ & $\mathbf{7 2}$ & $\mathbf{9}$ & $\mathbf{1 8 2}$ & $\mathbf{1 0 0}$ \\
\hline
\end{tabular}

Table 17. Volume-corrected mollusc MNI estimates for dominant species for Squares B and C, Site 62.

\begin{tabular}{|c|c|c|c|c|c|c|c|c|c|}
\hline \multirow{2}{*}{\multicolumn{2}{|c|}{ Square B }} & SU1m ${ }^{3}$ & SU2 $m^{3}$ & Total & \multirow{2}{*}{\multicolumn{2}{|c|}{ Square C }} & SU1m ${ }^{3}$ & SU2m ${ }^{3}$ & Total \\
\hline & & 0.0425 & 0.0825 & 0.125 & & & 0.0425 & 0.0825 & 0.125 \\
\hline \multirow[t]{2}{*}{ All shell } & MNI & 332 & 0 & 332 & \multirow[t]{2}{*}{ All shell } & MNI & 182 & 0 & 182 \\
\hline & $\mathrm{MNI} / \mathrm{m}^{3}$ & 7812 & 0 & 2656 & & $\mathrm{MNI} / \mathrm{m}^{3}$ & 4282 & 0 & 1456 \\
\hline \multirow{2}{*}{$\begin{array}{l}\text { Anadara } \\
\text { trapezia }\end{array}$} & MNI & 17 & 0 & 17 & \multirow{2}{*}{$\begin{array}{l}\text { Anadara } \\
\text { trapezia }\end{array}$} & MNI & 10 & 0 & 10 \\
\hline & $\mathrm{MNI} / \mathrm{m}^{3}$ & 400 & 0 & 136 & & $\mathrm{MNI} / \mathrm{m}^{3}$ & 235 & 0 & 80 \\
\hline \multirow{2}{*}{$\begin{array}{l}\text { Pyrazus } \\
\text { ebeninus }\end{array}$} & MNI & 37 & 0 & 37 & \multirow{2}{*}{$\begin{array}{l}\text { Pyrazus } \\
\text { ebeninus }\end{array}$} & MNI & 28 & 0 & 28 \\
\hline & $\mathrm{MNI} / \mathrm{m}^{3}$ & 871 & 0 & 296 & & $\mathrm{MNI} / \mathrm{m}^{3}$ & 659 & 0 & 224 \\
\hline \multirow{2}{*}{$\begin{array}{l}\text { Saccostrea } \\
\text { spp. }\end{array}$} & MNI & 273 & 0 & 273 & \multirow{2}{*}{$\begin{array}{l}\text { Saccostrea } \\
\text { spp. }\end{array}$} & MNI & 142 & 0 & 142 \\
\hline & $\mathrm{MNI} / \mathrm{m}^{3}$ & 6424 & 0 & 2184 & & $\mathrm{MNI} / \mathrm{m}^{3}$ & 1721 & 0 & 1136 \\
\hline \multirow{2}{*}{$\begin{array}{l}\text { Trichomya } \\
\text { hirsuta }\end{array}$} & MNI & 3 & 0 & 3 & \multirow{2}{*}{$\begin{array}{l}\text { Conuber } \\
\text { sordidum }\end{array}$} & MNI & 2 & 0 & 2 \\
\hline & $\mathrm{MNI} / \mathrm{m}^{3}$ & 71 & 0 & 24 & & $\mathrm{MNI} / \mathrm{m}^{3}$ & 47 & 0 & 16 \\
\hline
\end{tabular}

Table 18. Mollusc weights (g) by XU for Square C, Site 62.

\begin{tabular}{|l|r|r|r|r|r|r|}
\hline \multicolumn{1}{|c|}{ Species } & \multicolumn{1}{c|}{$\mathbf{3}$} & \multicolumn{1}{c|}{$\mathbf{4}$} & \multicolumn{1}{c|}{$\mathbf{5}$} & \multicolumn{1}{c|}{$\mathbf{6}$} & \multicolumn{1}{c|}{$\begin{array}{c}\text { Total } \\
\text { (g) }\end{array}$} & \multicolumn{1}{c|}{ Weight } \\
\hline Anadara trapezia & 0 & 14.19 & 77.18 & 14.33 & 105.7 & 8.02 \\
\hline Conuber sordidum & 0 & 0 & 1.15 & 0 & 1.15 & 0.09 \\
\hline Pyrazus ebeninus & 17.48 & 183.93 & 320.74 & 18.10 & 540.25 & 40.98 \\
\hline Saccostrea spp. & 17.54 & 231.07 & 408.89 & 13.13 & 670.63 & 50.87 \\
\hline Indeterminate & 0 & 0 & 0 & 0.55 & 0.55 & 0.04 \\
\hline \multicolumn{1}{|c|}{ Total } & $\mathbf{3 5 . 0 2}$ & $\mathbf{4 2 9 . 1 9}$ & $\mathbf{8 0 7 . 9 6}$ & $\mathbf{4 6 . 1 1}$ & $\mathbf{1 3 1 8 . 2 8}$ & $\mathbf{1 0 0}$ \\
\hline
\end{tabular}

Table 19. Molluscan diversity measures for Squares B and C, Site 62.

\begin{tabular}{|l|r|r|}
\hline \multicolumn{1}{|c|}{ Diversity Measure } & \multicolumn{1}{c|}{ Square B } & \multicolumn{1}{c|}{ Square C } \\
\hline NTAXA & 6 & 4 \\
\hline Individuals & 332 & 182 \\
\hline Simpson's Index (1-D) & 0.3087 & 0.3644 \\
\hline Shannon Index of Diversity ('H) & 0.6351 & 0.6906 \\
\hline Shannon Index of Evenness (E) & 0.3545 & 0.4982 \\
\hline
\end{tabular}




\section{Taxon-Specific Analysis (Square C)}

Saccostrea spp.

Saccostrea spp. was the most abundant taxon in Square C, with a NISP of 917 (Table 15), and an MNI of 142 (Table 16). Heights were obtained for 50 valves, lengths for 52 valves, and depths for 39 valves; the descriptive statistics are detailed in Table 20. Single sample $t$ test results indicated statistically significant differences for all three variables (Table 21), perhaps related to the modal distribution. However, further non-parametric Kolmogorov-Smirnov testing (Table 22) and the size-frequency histograms (Figure 14) indicated normal distributions.

Four of the 49 valves $(8 \%)$ are juveniles or subadults as they measure between $9.69 \mathrm{~mm}$ and $15.85 \mathrm{~mm}$. As demonstrated in Figure 14, the majority of the valves from Square $\mathrm{C}$ fall into the $25-55 \mathrm{~mm}$ range with a peak at $35-$ $40 \mathrm{~mm}$ and a second, smaller, peak at $45-50 \mathrm{~mm}$ suggesting that the shellfish were harvested from a relatively mature population not subject to over-exploitation. Three valves exceeded $60 \mathrm{~mm}$ in height. None of the 142 valves included in the MNI exhibited the elongated hinges or beaks indicative of 'old' oysters (Galtsoff 1964). Only five of the valves (0.1\%) exhibited evidence of being cemented to other oysters suggesting that, as at Site $75 \mathrm{~b}$, the bed from which they were obtained was not tightly packed or clustered. Twenty-eight valves $(19.71 \%)$ were cemented to eight $P$. ebeninus shells; one of the $P$. ebeninus was literally covered by seven Saccostrea spp. lower valves. While only three valves had flat bases suggesting they grew directly on rock or a firmly packed surface, ten $(7.04 \%)$ were noted to be deeply fluted or ribbed. The valves resemble the descriptions provided by Kent (1992:4) of sand and bed oysters. The mean valve depth is $12.96 \mathrm{~mm}$ and, as Figure 14 demonstrates, most of the valves are between $7.5 \mathrm{~mm}$ and $17.5 \mathrm{~mm}$ in depth. Taken with the high occurrence of valves attached to $P$. ebeninus shells the data suggest that although there was an adult (sexually mature) population of Saccostrea spp. provisioning the area around Site 62, it was not growing in clusters or reefs and was instead based around firm substrates and Hercules club whelk shells for support. The fragmentation ratio for Saccostrea was 6.45, considerably lower than that for Site $75 \mathrm{~b}$. None of the shells exhibited evidence of burning.

\section{Pyrazus ebeninus}

$P$. ebeninus was the second most abundant species in Square C, with a NISP of 78 and MNI of 28 (Tables 15-16). The breakage patterns observed were similar to those at Site $75 \mathrm{~b}$. Only two complete specimens were recovered, rendering statistical analyses of shell height impossible. The specimens were $59.66 \mathrm{~mm}$ and $60.08 \mathrm{~mm}$ in height, within the range at which sexual maturity is reached (Vohra 1965). The fragmentation ratio was low at 2.78. Meehan (1982) noted that Telescopium and Terebralia specimens took three to four minutes to cook on a fire, before the shells were broken to extract the meat. This short exposure to heat does not leave evidence of burning (see also Robins and Stock 1990), and none was observed on the Square C P. ebeninus shells.

\section{Anadara trapezia}

The A. trapezia MNI was surprisingly low at 10 , with a NISP of 28. Although length measurements were obtained from 11 valves, this sample was too small for a statistically valid analysis. The lengths ranged from $20.4 \mathrm{~mm}$ to $40.75 \mathrm{~mm}$ with a mean value of $32.98 \mathrm{~mm}$ (std. dev. 5.47), placing the majority in the range of sexually mature adults. The fragmentation ratio was low at 2.8. None of the shells exhibited evidence of burning.

\section{Other Molluscan Species}

Two Conuber sordidum were recovered. The shell heights were $8.04 \mathrm{~mm}$ and $15.33 \mathrm{~mm}$, representing a juvenile and a subadult. Neither of the shells exhibited evidence of burning.

\section{Crustacean Remains (Square C)}

One plate of a crustacean of the class Cirripedia (barnacle) weighing $0.09 \mathrm{~g}$ was recovered.

\section{Vertebrate Remains (Square C)}

One Sillago ciliata (summer whiting) otolith $8.05 \mathrm{~mm}$-long and $0.09 \mathrm{~g}$ in weight was recovered from XU2. McNiven (1990a:206, 1991a:100) recorded an unburnt summer whiting otolith weighing $0.10 \mathrm{~g}$ from XU2 in Square B. Given the similarity in weights it is likely that the two form a pair. As for Site 75b, McNiven (1990a:206, 1991a:100) expressed doubts about the discard of the otolith, as recent dingo scats containing fish bones had been observed near the site. As at Site $75 \mathrm{~b}$, the otolith did not exhibit obvious evidence of chewing or gastric etching, but it was unworn, suggesting the possibility of primary rather than secondary deposition.

In Square B, $0.15 \mathrm{~g}$ of bone (including the otolith, the only identifiable element) was recovered, and in Square A (the only faunal analysis on that square) there were four fragments of bone $(0.77 \mathrm{~g})$ - one from a macropod long bone $(0.32 \mathrm{~g})$, and one burnt and calcined piece perhaps from a mammal $(0.26 \mathrm{~g})$ (McNiven 1991a). In comparison, 282 bone fragments totalling $1.92 \mathrm{~g}$ were recovered from Square C. Two fragments with a total weight of $0.01 \mathrm{~g}$ were unidentifiable, while 266 of the fragments $(1.83 \mathrm{~g})$ came from long bones of a small mammal or lizard, and the remaining 14 fragments $(0.08 \mathrm{~g})$ from ribs of a small mammal or lizard. All the bones were burned black, suggesting a non-dingo source of discard.

\section{Stone Artefacts (Square C)}

The stone assemblage from Square C comprised two flakes, 13 flaked pieces, and one core. Raw materials included silcrete $(n=11)$, quartz $(n=3)$, quartzite $(n=1)$, and one unidentified type. The assemblage was unremarkable (for details see Smith 2016) except for the core, which was a reused bevel-edged artefact. The core came from XU10, the excavation unit associated with the date of $824 \mathrm{cal} \mathrm{BP}$, and below the main midden deposit. McNiven (1991a:100) recorded 12 bevel-edged tools and 10 bevel flakes in a deflated assemblage on the intertidal flats adjacent to Site 62, which he considered to be robust evidence for plant food processing at the site.

\section{Discussion}

The data presented above have demonstrated that Aboriginal people have been using the eastern periphery of Tin Can Inlet for at least the past 900 years. However, the faunal assemblages from Sites 62 and 75b presented here indicate specialised (albeit varied) use of estuarine marine resources 
Table 20. Descriptive statistics for Saccostrea spp. for Square C, Site 62.

\begin{tabular}{|c|c|c|r|r|r|r|}
\hline Attribute & \multicolumn{1}{c|}{$\mathbf{~ N}$} & \multicolumn{1}{c|}{ Range } & \multicolumn{1}{c|}{ Minimum } & \multicolumn{1}{c|}{ Maximum } & \multicolumn{1}{c|}{ Mean } & \multicolumn{1}{c|}{ Std. Deviation } \\
\hline Valve height $(\mathrm{mm})$ & 50 & 57.48 & 9.69 & 67.17 & 39.4686 & 12.89428 \\
\hline Valve length $(\mathrm{mm})$ & 52 & 53.09 & 5.50 & 58.59 & 27.0594 & 11.44094 \\
\hline Valve depth $(\mathrm{mm})$ & 39 & 20.49 & 2.54 & 23.03 & 12.9677 & 4.67346 \\
\hline
\end{tabular}

Table 21. Single sample $t$ test results for Saccostrea spp. for Square C, Site 62.

\begin{tabular}{|c|c|c|c|c|c|c|}
\hline \multicolumn{7}{|c|}{ One-Sample Test } \\
\hline & \multicolumn{6}{|c|}{ Test Value $=0$} \\
\hline & \multirow[t]{2}{*}{$\mathbf{t}$} & \multirow[t]{2}{*}{ df } & \multirow[t]{2}{*}{ Sig. (2-tailed) } & \multirow[t]{2}{*}{$\begin{array}{c}\text { Mean } \\
\text { Difference }\end{array}$} & \multicolumn{2}{|c|}{$\begin{array}{l}95 \% \text { Confidence Interval } \\
\text { of the Difference }\end{array}$} \\
\hline & & & & & Lower & Upper \\
\hline Valve height & 21.644 & 49 & .000 & 39.46860 & 35.8041 & 43.1331 \\
\hline Valve length & 17.055 & 51 & .000 & 27.05942 & 23.8742 & 30.2446 \\
\hline Valve depth & 17.328 & 38 & .000 & 12.96769 & 11.4527 & 14.4827 \\
\hline
\end{tabular}

Table 22. Kolmogorov-Smirnov results for Saccostrea spp. for Square C, Site 62.

\begin{tabular}{|c|c|c|c|c|}
\hline \multicolumn{5}{|c|}{ One-Sample Kolmogorov-Smirnov Test } \\
\hline & & Valve Height & Valve Length & Valve Depth \\
\hline \multicolumn{2}{|l|}{$\mathrm{N}$} & 50 & 52 & 39 \\
\hline \multirow[t]{2}{*}{ Normal Parameters ${ }^{\mathrm{a}, \mathrm{b}}$} & Mean & 39.4686 & 27.0594 & 12.9677 \\
\hline & Std. Deviation & 12.89428 & 11.44094 & 4.67346 \\
\hline \multirow[t]{3}{*}{ Most Extreme Differences } & Absolute & .086 & .114 & .068 \\
\hline & Positive & .069 & .114 & .051 \\
\hline & Negative & -.086 & -.084 & -.068 \\
\hline \multicolumn{2}{|l|}{ Test Statistic } & .086 & .114 & .068 \\
\hline \multicolumn{2}{|l|}{ Asymp. Sig. (2-tailed) } & $.200^{\mathrm{c}, \mathrm{d}}$ & $.087^{\mathrm{c}}$ & $.200^{\mathrm{c}, \mathrm{d}}$ \\
\hline \multicolumn{5}{|l|}{ a. Test distribution is Normal. } \\
\hline \multicolumn{5}{|l|}{ b. Calculated from data. } \\
\hline \multicolumn{5}{|c|}{ c. Lilliefors Significance Correction. } \\
\hline \multicolumn{5}{|c|}{ d. This is a lower bound of the true significance. } \\
\hline
\end{tabular}



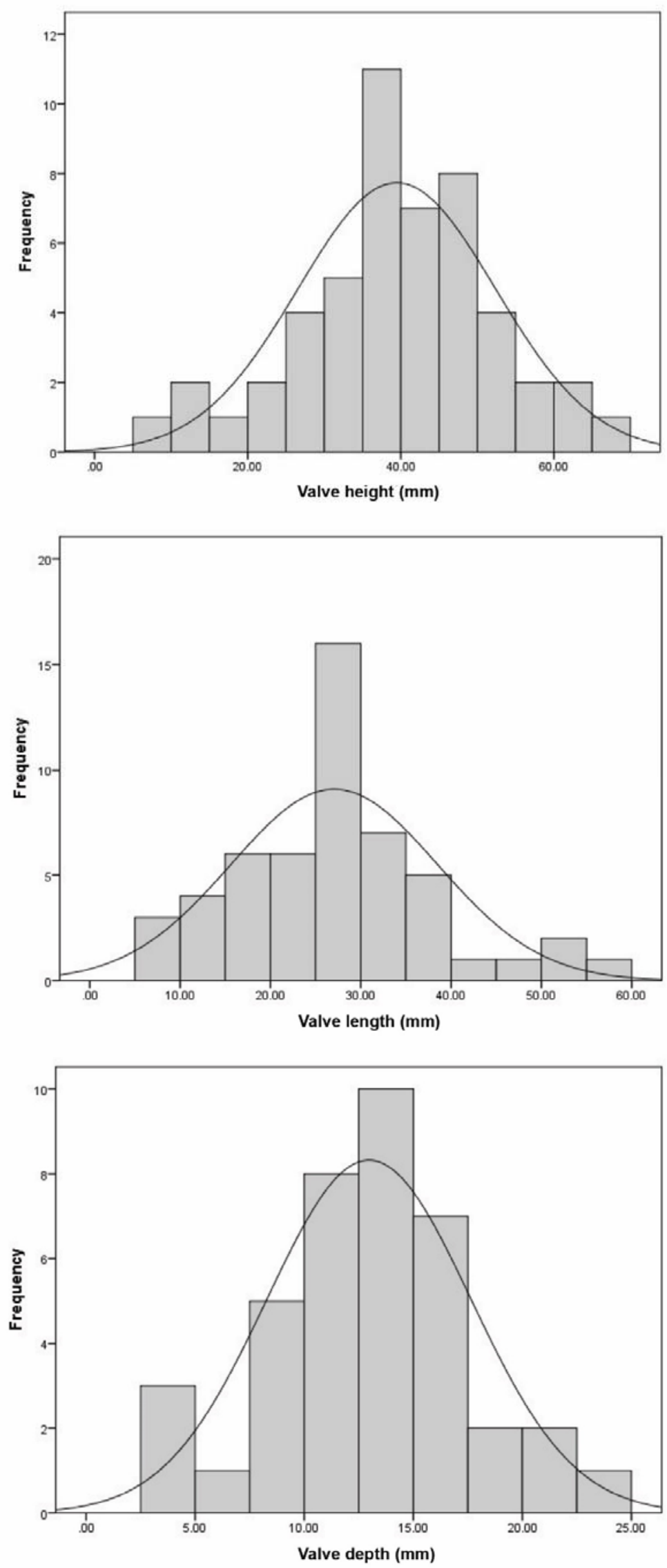

Figure 14. Histograms of Saccostrea spp. size frequency plotted against normal distribution curve for valve height, length and depth, Square C, Site 62. 
only within the past $200-400$ years. The following discussion elaborates on the broader implications of these chronological and spatial differences in patterns of subsistence and settlement within the context of development of estuarine resources in the inlet over the past 8000 years.

\section{Why a Subsistence Specialisation on Shellfish Across Tin Can Inlet?}

The presence of marine shellfish in Sites 62 and $75 \mathrm{~b}$ is not unexpected given the close proximity of estuarine shellfish beds to both sites (see above) and nineteenth century records of Aboriginal people in the Great Sandy Region eating estuarine shellfish. As one late nineteenth century European observed, 'The waters of Sandy Strait, between Fraser's Island and the mainland, afforded plenty of food to the natives. Fish, crabs, and delicious small oysters are still plentiful' (The Vagabond 1886; see also Devitt 1979).

In marked contrast to marine shellfish, Sites 62 and $75 \mathrm{~b}$ exhibited minuscule amounts of vertebrate remains. As McNiven (1991a:102) observed, the acidity of the sediments is unlikely to account for the dearth of vertebrate remains from the sites, especially as more bone was recovered from Site 62 which exhibited more persistently acidic $\mathrm{pH}$ levels. Terrestrial vertebrate species in the region, and for the Wallum zone in general, are low in both diversity and abundance. In common with terrestrial vertebrate remains from other archaeological sites across Cooloola (McNiven 1990a), the paucity of terrestrial animal bones in Sites 62 and $75 \mathrm{~b}$ may simply relate to lack of local availability. As Dwyer et al. (1979a:81) remarked, 'the nutritional reward' for huntergatherers exploiting Cooloola's mammal resource base, particularly macropods, 'would have been scant'.

The general lack of fish bone in the assemblages is less easily explained. In some respects, poor recovery of fish bones may be methodological and relate to the use of $3 \mathrm{~mm}$ mesh sieves; smaller mesh sizes (e.g. $1 \mathrm{~mm}$ ) may have enhanced recovery (see Ross and Duffy 2000; Ross and Tomkins 2011; Ulm 2002b; Walters 1989; but see Vale and Gargett 2002 for a contrary view). McNiven (1985, 1991a:102) noted that fish bones were macroscopically absent in midden sections along the coastline of Tin Can Inlet, and suggested that the discard of fish remains may have been spatially and/or temporally separated from the discard of molluscan remains. In support of this hypothesis, McNiven (1991b:20-21) cited an 1836 record of an Aboriginal fishing camp south of Inskip Point that would have survived only until the next high tide. Clearly ethnographic analogies must be used cautiously, but Eipper (1841), Nique and Hartenstein (1841) and Petrie (1904) made no mention of Aboriginal use of differing locations for consumption of fish, shellfish, or terrestrial and avian fauna during the colonial period in the Moreton Bay region. The role of dingoes in the removal of fish and other vertebrate remains is acknowledged but their impact has yet to be adequately quantified (McNiven 1990b; Ulm 2002b; Walters 1984). The available evidence continues to support McNiven's (1991a) conclusion that the paucity of terrestrial animal and fish bones in Sites 62 and $75 \mathrm{~b}$ is a true reflection of minimal hunting and fishing by people camped at both sites.

This scenario is similar to those presented by Faulkner (2011, 2013) for the Point Blane Peninsula in Arnhem Land; by Clune and Harrison (2009) for Western Australia's Abydos Plain, and by Morrison $(2003,2015)$ for the Weipa area in Far
North Queensland. In all cases there is a focus on predictable shellfish resources with a corresponding limited representation of vertebrates, both terrestrial and marine. Molluscs in Tin Can Inlet present easily accessed and harvested resources that are both reliable and diverse, especially in an area with depauperate terrestrial large vertebrate populations. The molluscs also represent a high resource biomass compared to that of fish and terrestrial vertebrates, with their fecundity also a factor in their selection as a food resource (e.g. Faulkner 2013; Mannino and Thomas 2002; Rowland 1994). Generally speaking, grazing and filterfeeding molluscs have high total biomass and dense populations (Mannino and Thomas 2002:456) and, as Faulkner (2013:180) noted, 'ecological data suggest that molluscs, and particularly bivalves, are capable of yielding very high output under specific conditions'. In this instance. they represent the dominant prey species in a particular landform with differential landscape and resource structure largely devoid of other animal resources, and where resources were located in the coastal zone (see Bailey 1975:58; Faulkner 2013:180). The scarcity of terrestrial faunal remains suggests that a limited range of subsistence activities was undertaken at the sites (e.g. Faulkner 2013; McNiven 1989; Morrison 2003, 2015; Smith 2016; Walters et al. 1987), and reflects the depauperate terrestrial fauna associated with Wallum floristic communities. Similar depauperate terrestrial faunal communities exist in a number of coastal areas around Australia, and as Faulkner (2013:180) observed in relation to the Point Blane Peninsula, 'the comparative lack of hard skeletal elements, such as teeth or otoliths, suggests that vertebrate fauna may not have been as an important part of coastal economies compared with the more recent past.'

Clearly shellfishing would not have been the only subsistence activity undertaken at Sites 62 and $75 \mathrm{~b}$ - the people who lived around Tin Can Inlet could not survive on shellfish alone. The backed artefact from Site $75 \mathrm{~b}$ attests to the fact that other subsistence and/or manufacturing activities also took place at the site, as backed artefacts have no archaeological or ethnographic association with molluscan collection and processing. However, access to other resources, particularly freshwater and plant/vegetable foods, would allow a focus on shellfish as the predominant prey at least in the short-term (e.g. Bailey 1975; Bourke 2002; Bourke 2004; Clune and Harrison 2009; Faulkner 2013; Mannino and Thomas 2002; Meehan 1982; Morrison 2003, 2015).

\section{Why the Absence of Large Marine Vertebrates in the Middens?}

The lack of bone recovery from large marine vertebrates such as dugongs, dolphins and sea turtles is curious given the welldocumented occurrence of these animals in Tin Can Inlet and the large calorific windfall of each. Nineteenth century historical records for the Cooloola region (including Tin Can Inlet) fail to mention Aboriginal consumption of either dugongs, dolphins or sea turtles (McNiven 1992a). In contrast, nineteenth century records for K'gari and Great Sandy Strait document that Aboriginal people hunted dugongs (Devitt 1979:77; Lauer 1977:17; Viator 1879), dolphins (Flinders 1814:10; cf. Curtis 1841:69), and sea turtles (Devitt 1979:77), and feasted on occasional stranded whales (Anonymous 1874). Indeed, Aboriginal people were a 'prominent feature' and an 'institution' of the colonial dugong fishery of Hervey Bay (Viator 1879). As one nineteenth 
century European observed in relation to potential foods for Fraser Island Aboriginal people, 'the adjacent seas swarm with mullet, crabs, oysters, and dugong' (Anonymous 1875). Meston (1923) added that the Aboriginal people of K'gari 'had unlimited supplies of food, including dugong ("yoo-ungan"), turtle ("milbeer"), crabs ("nal-loor"), oysters ("teebarr"), and a great variety of splendid fish'. A visitor to the Bogimbah mission station on the west coast of K'gari in 1899 noted that Aboriginal people had hunted 27 turtles and 11 dugongs over the previous seven months (Anonymous 1899).

Yet of the hundreds of middens recorded across the Great Sandy Region, none from Cooloola have revealed remains of large marine vertebrates (McNiven 1990a), while on K'gari a few fragments of sea turtle bone have been recorded within middens at Waddy Point (McNiven, pers. obs.) and dugong bone has been found in a midden on the east coast between Happy Valley and Yidney (Morwood 1975). However it needs to be kept in mind that no middens have been recorded in detail or excavated on the west coast of K'gari which overlooks the dugong habitats of Great Sandy Strait and Hervey Bay. In contrast, reliable ethnohistorical and archaeological evidence exists for Aboriginal hunting and consumption of dugongs and sea turtles for the Moreton Bay region to the immediate south (for an overview see McNiven 2006:115-116). Nonetheless, as McNiven observed, the archaeological presence of dugong and sea turtles is meagre, with dugong bone recovered from only seven sites and sea turtle remains from only four sites in the Moreton Region. The reasons for this are most likely multi-facetted. Moreton and Ross (2011:67) state that traditional dugong hunting in the Gorenpul-Dandrabin community of Moreton Bay (Quandamooka) was strictly men's business, as was the butchery and sharing of the meat and fat, and that dugong hunting was a dangerous activity undertaken only at certain times of the year. Petrie (1904:67) also commented that 'Dugong were only to be caught at certain seasons'. The processing technique described (Petrie 1904:68) of cooking, filleting and distributing the dugong where it was brought to shore implies that skeletal elements may not have been taken back to home bases. The same applies to the processing of sea turtles (Petrie 1904:82-83; see Bourke 2004 for a discussion on the absence of dugong bones at Hope Inlet, Darwin). As noted above, absence of bone in Sites 62 and 75b from Tin Can Inlet cannot simply be put down to poor preservation. The absence may indicate that the animals were not hunted by occupants of the two sites over the past 400 years, although this does not mean that dugongs and turtles were not hunted and consumed while camping at other locations in the region. Environmental factors, including seagrass patterning, may have influenced the distribution of dugongs and sea turtles during the periods of occupation of Sites 62 and 75b (see below), but there may also have been context-specific social circumstances not conducive to such large-game hunting (see Bird et al. 2013). In contrast, marine subsistence focused on the collection of shellfish and to a much lesser extent fishing.

\section{Does Micro-Habitat Dynamism Explain Diverse Shellfishing Practices at Tin Can Inlet?}

The low diversity of shellfish species at Sites 62 and 75b, and most particularly the absence or minimal occurrence of $A$. trapezia and T. hirsuta, is surprising given the abundance of those taxa, most notably T. hirsuta, elsewhere in the region (e.g. Booral Shell Mound - Frankland 1990; Smith 2016). The absence is therefore unlikely to reflect taste preference, foraging choice, or differential processing and discard. The presence of the species elsewhere in the region suggests a relationship between environmental conditions and microhabitat structure affecting species distribution. For example, although $P$. ebeninus and $A$. trapezia inhabit broadly similar habitat zones, $P$. ebeninus are mobile surface detrital feeders and A. trapezia are less mobile burrowing filter feeders, suggesting sedimentary structure and input, as well as nutrient input, will likely have an impact in combination with salinity, water temperature, and wave energy. The absence of $T$. hirsuta indicates a lack of suitable structures on which to settle (Gosling 2003), and this is discussed further below. The species composition therefore appears to be closely related to highly localised environmental and ecological conditions prevailing during the periods of occupation.

Dredge et al. (1977) identified a low-saline wedge in the southern sections of Tin Can Inlet, and that the hydrology of this wedge was strongly influenced by the inflow of freshwater from creeks draining through the coastal sand plain fed by springs emanating from the western side of the Cooloola sandmass. They also noted extensive seagrass beds related to the stability of the local watershed, with a resultant lack of silt in the estuary (Dredge et al. 1977:11). Dredge et al.'s (1977) mangrove and seagrass map (see Figure 2) shows the intertidal zone at Poverty Point near the location of Site $75 \mathrm{~b}$ had seagrass beds but is devoid of mangroves, while Site 62 adjacent to the intertidal zone west of the mouth of Cameron Creek had neither. Fisheries Research Consultants (1994) reported that many of the seagrass beds recorded by Dredge et al. had contracted, become sparser, or disappeared entirely; local fishermen advised that the seagrass beds were always in a state of flux (Fisheries Research Consultants 1994:4-8). Factors affecting seagrass distribution include salinity, temperature, light exposure and intensity, currents, tidal exposure, sediment and nutrient characteristics, and flood events (Fisheries Research Consultants 1994:10; McKenzie and Campbell 2003; McKenzie et al. 2014). Mangroves similarly have a mosaic-like distribution affected by salinity levels, soil characteristics including drainage, and, importantly, latitude and temperature. Many of the mangrove species in the inlet are at or near the southern limits of their distribution (Fisheries Research Consultants 1994:116) and the areas in which they occur have also varied since regular monitoring commenced in the 1980s. It is clear therefore that Tin Can Inlet is a dynamic environment, and that this dynamism is reflected both in the shellfish taxa recovered from the archaeological deposits as well as more broadly in terms of the modern occurrence of these taxa. Fisheries Research Consultants (1994:25) identified three factors influencing the distribution and abundance of molluscs as depth, sediment character, and the presence/absence of seagrass beds and associated flora, while acknowledging that the distribution and biology of the macro-molluscs of the region remain poorly known. What is apparent from the Fisheries Research Consultants' report is that the archaeological molluscan evidence accurately reflects the occurrence, distribution, and abundance of molluscan species in the adjacent intertidal areas.

The substrates in the Great Sandy Strait and Tin Can Inlet are mostly soft and range from silts and muds to coarse shelly sands (Fisheries Research Consultants 1994:25). These 
substrates are not typically associated with T. hirsuta or Isognomon spp. which require hard surfaces (rocks or mangrove roots) on which to attach themselves, and these taxa were absent during the Fisheries Research Consultants survey although mangroves were recorded. Generally speaking, there was a high correspondence between species diversity and abundance, and the presence of seagrass beds. However, $A$. trapezia were observed to occur more commonly in bare substrates or beds of Cymodocea serrulata than in beds of the dominant Zostera capricorni, the density of which may prevent spatfall larvae from penetrating the sediments and settling. Detrital-feeding whelks and carnivorous gastropods were present in all seagrass beds. The distribution and abundance of Saccostrea spp. was noted to be linked to the availability of suitable substrates on which spatfall could settle and attach, and that mud whelks commonly served this purpose (Fisheries Research Consultants 1994:27). In the late 1880 s it was observed that 'a very heavy fall of spat' had taken place on banks previously without oysters within Tin Can Inlet over a two year period following floods in 1887 (Fison 1889). Fison (1889) 'noticed young oysters of at least four or five different ages, the oldest not more than about twelve months, all on the one bank'. In 1914, oyster banks in Tin Can Inlet 'were being destroyed through "sanding up", i.e., being covered with sand being driven in by wind and waves" (Anonymous 1914). As suggested above, the molluscan assemblages of Sites 62 and $75 \mathrm{~b}$ are therefore accurate reflections of the localised dynamics of micro-habitats. The juvenile mussels at Site $75 \mathrm{~b}$ most likely represent tidallytransported isolated spat that managed to settle, but the local conditions did not support development of a viable colony. Similarly, the isolated occurrence of Isognomon spp. suggests a change in local environmental conditions around 200 years ago that allowed the establishment of a small but viable population for a brief period.

The minimal occurrence of $A$. trapezia in both sites indicates that during the periods of occupation the adjacent seagrass beds were not conducive to settlement of larvae and establishment of colonies. The absence of hard substrates for development of oyster reefs is indicated by the morphology of the oyster shells as well as the high proportion of valves attached to P. ebeninus shells.

\section{Why are the Sites so Recent?}

Various environmental and social factors have contributed to the restricted chronologies of Sites 62 and $75 \mathrm{~b}$ to $<1000-1500$ years ago. As detailed above, a broad range of palaeoenvironmental evidence indicates that not only did the sea arrive in the Great Sandy Region around 8000 years ago, but so did estuarine resources such as shellfish. As such, the onset of midden formation at Sites 62 and $75 \mathrm{~b}$ at 165 and 340 cal BP respectively cannot be put down to recent development of shellfish resources. Indeed, these midden deposits developed some 7000 years after estuarine shellfish became established in the region. In the Moreton Bay region to the south, varying dates for the establishment of estuarine middens have been linked to staggered development of estuarine resources over the past 5000 years (e.g. St. Helena Island - Alfredson 1983:84; Hope Island - Walters et al. 1987; Sandstone Point - Hall 1999:175) and for Moreton Bay more broadly (e.g. Hall 1982:92, 1999; Morwood 1987:343; Walters 1992:176). In the case of late Holocene estuarine middens, increased availability of estuarine resources was tied to independent geomorphological evidence (e.g. Flood 1981; Hekel et al. 1979) for a fall in sea levels and associated coastal progradation over the past 2000-3000 years. Such resource development interpretations break down in the case of estuarine middens dating to the past 1000 years where delayed onset of estuarine resources for 7000 years after arrival of the sea is implausible. For a number of archaeologists, the development of late Holocene estuarine middens has been linked to local demographic changes and increased social demands on marine resources in terms of fish (e.g. Sandstone Point - Nolan 1986; Walters 1989; cf. Ulm 2002b) and shellfish (e.g. Maroochy River mouth - McNiven 1989; Tin Can Inlet - McNiven 1991a). More recently, Smith (2016:219) observed that while the intensification noted for the late Holocene is reflected in an increased number of sites across the region, low site accumulation rates are maintained and there are minimal resource impacts. She considered this may be evidence of population variability linked with an increased resource base (in terms of area, but not in basic character), changing foraging patterns, and socio-economic reorganisation in a complex, non-linear pattern in the more recent past.

The distinct chronological phases of resource use at Sites 62 and 75b, and the implications of increased exploitation of local marine resources in the past 200-300 years, were interpreted by McNiven (1991a, 1999, 2006) in part as evidence of social fissioning and secondary augmentation of Recent Phase developments during the early European contact period in the mid-to-late nineteenth century. While these post-contact developments cannot be dismissed, the date of $340 \mathrm{cal} \mathrm{BP}$ for the onset of midden formation at Site $75 \mathrm{~b}$ indicates pre-European exploitation of the estuarine resources. The dynamic environment of the inlet is also a consideration, but the tidal regimes were well established by 800-900 years ago (Ryan et al. 2003; Smith 2016). The molluscan data presented above do not demonstrate signs of over-exploitation or resource depression (Claassen 1998; Faulkner 2013; Mannino and Thomas 2002; Smith 2016), suggesting that if there were recent increased exploitation of marine resources, it was of a scale insufficient to leave demonstrable physical traces at these two sites.

A terrestrial environmental dimension that may be critical to understanding the recent antiquity of Sites 62 and $75 \mathrm{~b}$ is landform development. As mentioned above, the midHolocene sea level highstand would have inundated all areas along the eastern margins of Tin Can Inlet currently $<1.5 \mathrm{~m}$ above current sea level between $\sim 7000$ and at least 2000 years ago. It is only with rapid sea level fall over the past 1000 2000 years that low elevation areas such as Sites 62 and $75 b$ would have become available for occupation. In this sense, Sites 62 and $75 \mathrm{~b}$ cannot be used as an indicator of when Aboriginal people first began to occupy and use the eastern margins of Tin Can Inlet. Such early evidence would only potentially be available in higher elevation ( $>5 \mathrm{~m}$ ASL) sand dunes that would have continued to be exposed during the mid-Holocene highstand. Such areas can be found in patches not along the current coastline but further inland across the coastal sand plains that are today hundreds of metres and even kilometres inland. This issue of inland mid-Holocene shorelines was discussed in detail by Hall and Lilley (1987) in relation to the New Brisbane Airport site: Ulm (2011:455) later commented 'The simplest explanation for the low number of coastal sites dating to before the late Holocene is 
that the majority of extant coastal landscapes only became available for occupation in this period'. This point has also been made forcefully more recently by Robins et al. (2015:204) who note that 'Knowledge of landscape history is also prerequisite to finding older sites, the identification of which is essential if cultural chronologies are to be based on a more representative sample of archaeological sites'. Indeed, this paper concurs with these views with the estimated $<1000-1500$ year age of Sites 62 and $75 b$ associated in part with the likely formation of associated landforms with sea level fall over the past 2000 years. In terms of this paper, it is clear that future archaeological research aimed at documenting pre-2000 year old sites along Tin Can Inlet will need to focus on higher elevation $(>5 \mathrm{~m})$ dunes located inland across the coastal sand plain and adjacent to what would have been the mid-Holocene highstand palaeoshoreline. Until these locations have been sampled for archaeological materials, McNiven's (1991a:103) contention that Tin Can Inlet witnessed a major increase in site activity within the past 1000 years remains hypothetical.

Another terrestrial environmental dimension that may have relevance to the late occupation of Sites 62 and $75 \mathrm{~b}$ is vegetation change. Although direct chronological correlations are lacking from the available environmental reconstructions for K'gari, there is evidence of a decrease in rainforest Araucarian species around Lake Allom at $\sim 450$ BP (Donders et al. 2006), and an increase in dry sclerophyll species, Myrtaceae (including Eucalyptus spp.), Casuarinaceae, Leptospermum, Melaleuca, Poaceae, and Callitris in the upper levels of the Wathumba and Moon Point fenland cores over the past 500 years or so (Moss et al. 2016). Taken together, these studies may indicate an increase in the mixed woodland and forest vegetation zones which now occur on the eastern periphery of Tin Can Inlet, and which have demonstrated a high correlation with sites containing midden deposits. While there may have been an associated impact on the development of favourable habitats for the molluscan species reflected in the middens, new vegetation patterns may have increased the attractiveness of the eastern margins of Tin Can Inlet as a place to establish longer-term residential campsites. The change in the vegetation structure may have allowed a move from more specialised resource-based ephemeral occupation based around plant exploitation in the rainforest/swamp areas evidenced by the bevel tools in the deflated artefact assemblage, and the reused bevel tool from Site 62, to more intensive use of the marine/estuarine resources fostered by more favourable camping or longerterm residential loci.

\section{Conclusion}

This paper has shown the potential value of an historical ecology approach to southeast Queensland coastal archaeology. Employing the premise that socio-cultural elements, archaeological economic data, and environmental and ecological data are inextricably linked (e.g. Milner 2013; Thompson 2013) has allowed a more holistic approach than the previous studies permitted. Detailed understanding of the habitat requirements of different species of shellfish found within midden Sites 62 and 75 b provided new insights into potential reasons for similarities and differences between the two shellfish assemblages. Equally important, an historical ecology perspective provides invigorated scope to further explore the potential effects of Aboriginal land- and sea-use practices on altering the nature and availability of estuarine and swamp resources. In this sense, the full explanatory potential of the dynamic and recursive relationship between Aboriginal people and estuarine resources in understanding the long-term development of midden deposits in southeast Queensland has yet to be realised.

In addition to the identification of old coastal landforms away from the present coastline and exploration of their archaeological potential, further research is required on potential chronological changes in the development of estuarine resources in different regions of southeast Queensland since arrival of the sea $\sim 8000$ years ago. In particular, further research is required to test the hypothesis that estuarine resources did increase in availability within the past 2000-3000 years. Yet as Nolan (1986:97-98) pointed out, care needs to be taken not to create circular arguments whereby the late development of estuarine middens is taken as proxy evidence for the late development of estuarine resources.

Finally, this study has demonstrated the utility of the reanalysis of existing assemblages and datasets. Questions can still be asked of data derived from appropriately accessioned assemblages when coupled with contemporary approaches to analysis including palaeoenvironmental reconstructions, morphometric analyses of molluscs, and statistical analyses.

\section{Acknowledgements}

Excavation of Sites 62 and 75b in 1985 was made possible by the generous assistance provided to Ian McNiven by Bryce Barker, Kathy Frankland, Alicia Jamieson, Scean Kearns, Miles Kemp, Fiona Noble and Anna Nolan. The Queensland Museum Aboriginal and Torres Strait Islander Consultative Committee gave permission for the reanalysis of archaeological assemblages from coastal southeast Queensland as part of Smith's PhD research. We thank John Healy (Curator, Malacology, Natural Environments) for his expert advice, and Brit Asmussen (Senior Curator, Archaeology, Indigenous Culture, Cultures and Histories), Nick Hadnutt (Curator and formerly Collections Manager, Cultures and Histories), and Assistant Collection Managers Peter Volk and David Parkhill (Cultures and Histories) for access to the archaeological collections. The School of Social Science, The University of Queensland, provided laboratory facilities and technical support. The research was in part supported by Smith's Australian Postgraduate Award. Tam Smith particularly thanks Patrick Faulkner (University of Sydney, formerly of The University of Queensland) for his research guidance and friendship, and for his assistance with the statistical analyses. Elaine Brown generously provided historical information on the Figure 5 photographs. Sean Ulm (James Cook University) provided advice on $\Delta \mathrm{R}$ values for radiocarbon dating. Kara Rasmanis (Monash University) drafted Figure 1 . We thank the three anonymous reviewers whose comments helped to sharpen aspects of the paper and clarify our arguments.

\section{References}

Ainis, A.F., R.L. Vellanoweth, Q.G. Lapeña and C.S. Thornber 2014 Using non-dietary gastropods in coastal shell middens to infer kelp and seagrass harvesting and paleoenvironmental conditions. Journal of Archaeological Science 49:343-360. https://doi.org/10.1016/ j.jas.2014.05.024

Alfredson, G. 1983 St Helena Island - A changing pattern of exploitation? Australian Archaeology 17:79-86.

Anonymous 1870 Down the coast. Brisbane Courier 30 September, p.3.

Queensland Archaeological Research | Vol. 22 | 2019 | 31 
Anonymous 1873 Colonial topics: Dugong fishery. Northern Argus 27 September, p.3.

Anonymous 1874 Article. Maryborough Chronicle 5 November, p.2.

Anonymous 1875 The Wide Bay and Burnett district. Maryborough Chronicle 23 October, pp.4-5.

Anonymous 1876 Dugong fishing in Queensland. Illustrated Adelaide News 1 November, p.7.

Anonymous 1877 Article. The Queenslander 30 June, p.6.

Anonymous 1880 Local and general. Maryborough Chronicle 1 June, p.2.

Anonymous 1888 Local news. Maryborough Chronicle 1 May, p.2.

Anonymous 1896 General news. Maryborough Chronicle 5 November, p.2.

Anonymous 1899 "The poor blacks." Aboriginal station at Fraser's Island. A recent visit. Brisbane Courier 22 April, p.4.

Anonymous 1914 Oyster banks at Tin Can. Maryborough Chronicle 27 January, p.5.

Atahan, P., H. Heijnis, J. Dodson, K. Grice, P. Le Métayer, K. Taffs, S. Hembrow, M. Woltering and A. Zawadzki 2015 Pollen, biomarker and stable isotope evidence of late Quaternary environmental change at Lake McKenzie, southeast Queensland. Journal of Paleolimnology 53(1):139-156. https://doi.org/10.1007/s10933-0149813-3

Bailey, G.N. 1975 The role of molluscs in coastal economies: The results of midden analysis in Australia. Journal of Archaeological Science 2(1):45-62. https://doi.org/10.1016/0305-4403(75)90045-X

Barr, C., J. Tibby, J.C. Marshall, G.B. McGregor, P.T. Moss, G.P. Halverson and J. Fluin 2013 Combining monitoring, models and palaeolimnology to assess ecosystem response to environmental change at monthly to millennial timescales: The stability of Blue Lake, North Stradbroke Island, Australia. Freshwater Biology 58(8):1614-1630. https://doi.org/10.1111/fwb.12154

Barry D.H. and P.R. Campbell 1979 A survey of the mammals and herptiles of Fraser Island, with comments on the Cooloola Peninsula, North Stradbroke, Moreton and Bribie Islands. In P.K. Lauer (ed.), Fraser Island, pp.147-177. Occasional Papers in Anthropology 8. St Lucia, QLD: Anthropology Museum, University of Queensland.

Beumer, J. and I. Halliday 1994 Effects of Habitat Disturbance on Coastal Fisheries Resources of Tin Can Bay/Great Sandy Strait. Brisbane: Fisheries Services, Queensland Department of Primary Industries.

Bird, D.W., B.F. Codding, R. Bliege Bird, D.W. Zeanah and C.J. Taylor 2013 Megafauna in a continent of small game: Archaeological implications of Martu camel hunting in Australia's Western Desert. Quaternary International 297:155-166. https://doi.org/10.1016/j.quaint.2013.01.011

Bourke, P.M. 2004 Three Aboriginal shell mounds at Hope Inlet: Evidence for coastal, not maritime late Holocene economies on the Beagle Gulf mainland, northern Australia. Australian Archaeology 59:10-22. https://doi.org/10.1080/03122417.2004.11681787

Bourke, T. 2002 Shell mounds and stone axes: Prehistoric resource procurement strategies at Hope Inlet, northern Australia. Bulletin of the Indo-Pacific Prehistory Association 6:35-44.

Bowen, G. 1998 Towards a generic technique for dating stone fish traps and weirs. Australian Archaeology 47:39-43. https://doi.org/ $\underline{10.1080 / 03122417.1998 .11681606}$

Braje, T. 2007 Archaeology, Human Impacts, and Historical Ecology on San Miguel Island, California. Unpublished PhD thesis, Department of Anthropology, University of Oregon, Eugene.
Braje, T.J. and J.M. Erlandson 2009 Molluscs and mass harvesting in the middle Holocene: Prey size and resource ranking on San Miguel Island, Alta California. California Archaeology 1(2):269290. https://doi.org/10.1179/cal.2009.1.2.269

Braje, T.J., D.J. Kennett, J.M. Erlandson and B.J. Culleton 2007 Human impacts on nearshore shellfish taxa: A 7,000 year record from Santa Rosa Island, California. American Antiquity 72(4):735756. https://doi.org/10.2307/25470443

Braje, T.J., T.C. Rick, R.L. DeLong and J.M. Erlandson 2011 Archaeology and historical ecology of California Channel Island marine mammals. In T.J. Braje and T.C. Rick (eds), Human Impacts on Seals, Sea Lions, and Sea Otters: Integrating Archaeology and Ecology in the Northeast Pacific, pp.273-296. Berkeley: University of California Press.

Braje, T.J., T.C. Rick and J.M. Erlandson 2012 A trans-Holocene historical ecological record of shellfish harvesting on California's Northern Channel Islands. Quaternary International 264:109-120. https://doi.org/10.1016/j.quaint.2011.09.011

Brown, E. 1999 Colonial enterprise: Pettigrew and Sim's Dundathu sawmill, 1862-1893. In J. Dargavel and B. Libbis (eds), Australia's Ever-Changing Forests: Proceedings of the Fourth National Conference on Australian Forest History, pp.239-246. Canberra: Centre for Resource and Environmental Studies, Australian National University, and Australian Forest History Society.

Brown, E. 2000 Cooloola Coast: Noosa to Fraser Island. The Aboriginal and Settler Histories of a Unique Environment. St Lucia, QLD: University of Queensland Press.

Brown, E. 2004 William Pettigrew 1825-1906. Sawmiller, Surveyor, Shipowner and Citizen: An Immigrant's Life in Colonial Queensland. Unpublished PhD thesis, University of Queensland, Brisbane.

Bruinsma, C. and K. Danaher 2000 Queensland Coastal Wetland Resources: Round Hill Head to Tin Can Inlet. Brisbane: Queensland Department of Primary Industries.

Buroker, N.E., W.K. Hershberger and K.K. Chew 1979 Population genetics of the Family Ostreidae. I. Intraspecific studies of Crassostrea gigas and Saccostrea commercialis. Marine Biology 54(2):157-169. https://doi.org/10.1007/BF00386595

Cagnazzi, D.D.B., P.L. Harrison, G.J.B. Ross and P. Lynch 2011 Abundance and site fidelity of Indo-Pacific Humpback dolphins in the Great Sandy Strait, Queensland, Australia. Marine Mammal Science 27(2):255-281. https://doi.org/10.1111/j.1748-7692.2009. $\underline{\text { 00296.x }}$

Carpenter, C.E. and V.H. Niem 1998 The Living Marine Resources of the Western Pacific. Volume 1. Virginia: Old Dominion University Press.

Catterall, C.P. and I.R. Poiner 1987 The potential impact of human gathering on shellfish populations, with reference to some NE Australian intertidal flats. OIKOS 50(1):114-122. https://doi.org/ $\underline{10.2307 / 3565407}$

Claassen, C.L. 1998 Shells. Cambridge: Cambridge University Press.

Claassen, C.L. 2000 Quantifying shell: Comments on Mason, Peterson, and Tiffany. American Antiquity 65(2):415-418. https://doi.org/10.2307/2694068

Clune, G. and R. Harrison 2009 Coastal shell middens of the Abydos Plain, Western Australia. Archaeology in Oceania 44(S1):70-80. https://doi.org/10.1002/j.1834-4453.2009.tb00069.x

Cooley, P.J. 2017 Initiation and Growth of Mid-Holocene Coral Reefs, Cleveland Point, Moreton Bay, Queensland. Unpublished MAppSci thesis, Queensland University of Technology, Brisbane. 
Corkeron, P.J. 1995 Humpback whales (Megaptera novaeangliae) in Hervey Bay, Queensland: Behaviour and responses to whalewatching vessels. Canadian Journal of Zoology 73(7):1290-1299. https://doi.org/10.1139/z95-153

Cotter, M. 1996. Holocene environmental change in Deception Bay, southeast Queensland: A palaeogeographical contribution to MRAP Stage II. In S. Ulm, I. Lilley and A. Ross (eds), Australian Archaeology '95: Proceedings of the 1995 Australian Archaeological Association Annual Conference, pp.193-203. Tempus 6. St Lucia, QLD: Anthropology Museum, University of Queensland.

Crumley, C. 1994 (ed.) Historical Ecology: Cultural Knowledge and Changing Landscapes. Santa Fe, New Mexico: School of American Research.

Curtis, J. 1841 Shipwreck of the Stirling Castle. London: George Virtue.

Daley, B., P. Griggs and H. Marsh 2008 Exploiting marine wildlife in Queensland: The commercial dugong and marine turtle fisheries, 1847-1969. Australian Economic History Review 48(3):227-265. https://doi.org/10.1111/j.1467-8446.2008.00240.x

Devitt, J. 1979 Fraser Island: Aboriginal Resources and Settlement Pattern. Unpublished BA (Hons) thesis, Department of Anthropology and Sociology, University of Queensland, Brisbane.

Devoy, J.N. 1902 Narrative and Reminiscences of a Yachting Cruise in Sandy Straits and Hervey Bay, Queensland. Brisbane: Outridge Publishing Co. Ltd.

Donders, T.H., F. Wagner and H. Visscher 2006 Late Pleistocene and Holocene subtropical vegetation dynamics recorded in perched lake deposits on Fraser Island, Queensland, Australia. Palaeogeography, Palaeoclimatology, Palaeoecology 241(34):417-439. https://doi.org/10.1016/j.palaeo.2006.04.008

Dredge, M., H. Kirkman and M. Potter 1977 A Short-Term Biological Survey. Tin Can Inlet/Great Sandy Strait. CSIRO Division of Fisheries and Oceanography Report 68. Cronulla: Marine Laboratory.

Dwyer, P.D., M. Hockings and J. Willmer 1979a Mammals of Cooloola and Beerwah. Proceedings of the Royal Society of Queensland 90:65-84.

Dwyer, P.D., J. Kikkawa and G.J. Ingram 1979b Habitat relations of vertebrates in subtropical heathlands of coastal southeastern Queensland. In R.L. Specht (ed.), Ecosystems of the World 9A. Heathlands and Related Scrublands. Descriptive Studies, pp.281299. Amsterdam: Elsevier Scientific Publishing Co.

Eipper, C. 1841 Observations made on a journey to the natives at Toorbul, August 12, 1841. Colonial Observer 14 October, p.10.

Erlandson, J. and R. Colten 1991 Hunter-Gatherers of Early Holocene Coastal California. Los Angeles: Institute of Archaeology, University of California.

Erlandson, J. and M. Glassow 1997 Archaeology of the California Coast during the Mid-Holocene. Los Angeles: Institute of Archaeology, University of California.

Erlandson, J.M., T.C. Rick and T.J. Braje 2009 Fishing up the food web?: 12,000 years of maritime subsistence and adaptive adjustments on California's Channel Islands. Pacific Science 63(4):711-724. https://doi.org/10.2984/049.063.0411

Erlandson, J.M., T.C. Rick, T.J. Braje, A. Steinberg and R.L. Vellanoweth 2008 Human impacts on ancient shellfish: A 10,000 year record from San Miguel Island, California. Journal of Archaeological Science 35(8):2144-2152. https://doi.org/10.1016/ j.jas.2008.01.014
Erlandson, J.M., T.C. Rick, J.A. Estes, M.H. Graham, T.J. Braje and R.L. Vellanoweth 2005 Sea otters, shellfish, and humans: 10,000 years of ecological interaction on San Miguel Island, California. In D.K. Garcelon and C.A. Schwemm (eds), Proceedings of the 6th California Islands Symposium, pp.56-68. Arcata, CA: National Park Service Technical Publication CHIS-05-01, Institute for Wildlife Studies.

Erlandson, J.M., T.C. Rick and R. Vellanoweth 2004 Human impacts on ancient environments: A case study from California's Northern Channel Islands. In S.M. Fitzpatrick (ed.), Voyages of Discovery: The Archaeology of Islands, pp.51-83. Westport, CT: Praeger.

Erlandson, J.M., T.C. Rick, R.L. Vellanoweth and D.J. Kennett 1999 Marine subsistence at a 9300 year old shell midden on Santa Rosa Island, California. Journal of Field Archaeology 26(3):255-265. https://doi.org/10.1179/ifa.1999.26.3.255

Erlandson, J., M. Tveskov and R. Byram 1998 The development of maritime adaptations on the southern northwest coast of North America. Arctic Anthropology 35(1):6-22.

Evans, R. and J. Walker 1977 "These strangers, where are they going?" Aboriginal-European relations in the Fraser Island and Wide Bay region 1770-1905. University of Queensland Anthropology Museum, Occasional Papers in Anthropology 8:39-105.

Faulkner, P. 2006 The Ebb and Flow: An Archaeological Investigation of Late Holocene Economic Variability on the Coastal Margin of Blue Mud Bay, Northern Australia. Unpublished PhD thesis, The Australian National University, Canberra.

Faulkner, P. 2008 Patterns of chronological variability in occupation on the coastal margin of Blue Mud Bay. Archaeology in Oceania 43(2):81-88. https://doi.org/10.1002/j.1834-4453.2008.tb00033.x

Faulkner, P. 2010 Morphometric and taphonomic analysis of granular ark (Anadara granosa) dominated shell deposits of Blue Mud Bay, northern Australia. Journal of Archaeological Science 37(8):1942-1952. https://doi.org/10.1016/j.jas.2010.02.021

Faulkner, P. 2011 Late Holocene mollusc exploitation and changing near-shore environments: A case study from the coastal margin of Blue Mud Bay, northern Australia. Environmental Archaeology 16(2):137-150. https://doi.org/10.1179/174963111X1311080326 $\underline{0976}$

Faulkner, P. 2013 Life on the Margins: An Archaeological Investigation of Late Holocene Economic Variability, Blue Mud Bay, Northern Australia. Terra Australis 38. Canberra: ANU E Press.

Field, A. 2014 Discovering Statistics using IBM SPSS Statistics: And Sex and Drugs and Rock ' $n$ ' Roll. 4th ed. Los Angeles: Sage.

Fisheries Research Consultants 1993 A Study of Marine and Intertidal Habitats of the Great Sandy Region. Wellington Point, QLD: Fisheries Research Consultants.

Fisheries Research Consultants 1994 Establishment of Long-Term Seagrass Monitoring Sites within the Great Sandy Strait and Tin Can Inlet. Wellington Point, QLD: Fisheries Research Consultants.

Fison, C.S. 1889 Report on the oyster fisheries of Moreton Bay and Great Sandy Island Strait. Queensland Votes and Proceedings 3:937-941.

Flinders, M. 1814 A Voyage to Terra Australis. Vol. 2. London: G. and W. Nicol.

Flood, P.G. 1981 Carbon-14 dates from the coastal plains of Deception Bay, southeastern Queensland. Queensland Government Mining Journal 81:19-23.

Foley, S. 1994 The Badtjala People. Hervey Bay, QLD: Thoorgine Educational and Cultural Centre Aboriginal Corporation Inc. 
Frankland, K. 1990 Booral: A Preliminary Investigation of an Archaeological Site in the Great Sandy Strait Region, South-East Queensland. Unpublished BA (Hons) thesis, Department of Anthropology and Sociology, The University of Queensland, Brisbane.

Frawley, J. 2017 Oyster culture in the estuary worlds of southern Queensland. RCC Perspectives: Transformations in Environment and Society 2017/2:11-17. https://doi.org/10.5282/rcc/7904

Galtsoff, P.S. 1964 The American Oyster Crassostrea virginica Gmelin. Fishery Bulletin of the Fisheries and Wildlife Service 64. Washington, D.C.: United States Department of the Interior, Fish and Wildlife Service, Bureau of Commercial Fisheries.

Gillies, C.L., I.M. McLeod, H.K. Alleway, P. Cook, C. Crawford, C. Creighton, B. Diggles, J. Ford, P. Hamer, G. Heller-Wagner, E. Lebrault, A. Le Port, K. Russell, M. Sheaves and B. Warnock 2018 Australian shellfish ecosystems: Past distribution, current status and future direction. PloS ONE 13(2):e0190914. https://doi.org/10.1371/ journal.pone.0190914

Gillieson, D.S. and J. Hall 1982 Bevelling bungwall bashers: A usewear study from southeast Queensland. Australian Archaeology $14: 43-61$.

Giovas, C.M. 2009 The shell game: Analytic problems in archaeological mollusc quantification. Journal of Archaeological Science 36(7):1557-1564. https://doi.org/10.1016/j.jas.2009.03.017

Giovas, C.M., M. Clark, S.M. Fitzpatrick and J. Stone 2013 Intensifying collection and size increase of the tessellated nerite snail (Nerita tessellata) at the Coconut Walk site, Nevis, northern Lesser Antilles, AD 890-1440. Journal of Archaeological Science 40(11):4024-4038. https://doi.org/10.1016/j.jas.2013.05.008

Giovas, C.M, S.M. Fitzpatrick, M. Clark and M. Abed 2010 Evidence for size increase in an exploited mollusc: Humped conch (Strombus gibberulus) at Chelechol ra Orrak, Palau, from ca. 3,0000 BP. Journal of Archaeological Science 37(11):2788-2798. https://doi.org/10.1016/j.jas.2010.06.013

Glassow, M.A. 2000 Weighing vs. counting shellfish remains: A comment on Mason, Peterson, and Tiffany. American Antiquity 65(2):407-414. https://doi.org/10.2307/2694067

Gosling, E. 2003 Bivalve Molluscs: Biology, Ecology and Culture. Oxford: Blackwell Publishing.

Grimes, K.G. 1992a Fraser Island, Queensland. Sheet SG 56-3 (Part) International Index. 1:250,000 Geological Series Explanatory Notes. Brisbane: Department of Resource Industries, Geological Survey of Queensland.

Grimes, K.G. 1992b Shallow Stratigraphic Drilling in the Wide Bay and Maryborough 1:250 000 Sheet Areas. Queensland Resource Industries Record 1991/30. Brisbane: Department of Resource Industries Queensland.

Hall, J. 1980 Minner Dint: A recent Aboriginal midden on Moreton Island, southeast Queensland. University of Queensland Anthropology Museum, Occasional Papers in Anthropology 10:94112.

Hall, J. 1982 Sitting on the crop of the bay: An historical and archaeological sketch of Aboriginal settlement and subsistence in Moreton Bay, southeast Queensland. In S. Bowdler (ed.), Coastal Archaeology in Eastern Australia: Proceedings of the 1980 Valla Conference on Australian Prehistory, pp.79-95. Occasional Papers in Prehistory 11. Canberra: Department of Prehistory, Research School of Pacific Studies, Australian National University.

Hall, J. 1984 Exploratory excavation at Toulkerrie midden (LB: B75), Moreton Island, S.E. Queensland. Queensland Archaeological Research 1:61-84. https://doi.org/10.25120/qar.1.1984.208
Hall, J. 1999 The impact of sea level rise on the archaeological record of the Moreton region, southeast Queensland. In J. Hall and I.J. McNiven (eds), Australian Coastal Archaeology, pp.169-184. Research Papers in Archaeology and Natural History 31. Canberra: Archaeology and Natural History Publications, Research School of Pacific and Asian Studies, Australian National University.

Hall, J. and G. Bowen 1989 An excavation of a midden complex at the Toulkerrie oystermans lease, Moreton Island, S.E. Queensland. Queensland Archaeological Research 6:3-27. https://doi.org/ 10.25120/qar.6.1989.135

Hall, J. and I. Lilley 1987 Excavation at the New Brisbane Airport site (LB:C69): Evidence for early mid-Holocene coastal occupation in Moreton Bay, SE Queensland. Queensland Archaeological Research 4:54-79. http://dx.doi.org/10.25120/qar.4.1987.172

Hammer, Ø. 2001 PAST PAleontological STatistics. Natural History Museum, University of Oslo, Oslo, Norway. Version 3.04.

Harris, M., M. Weisler and P. Faulkner 2015 A refined protocol for calculating MNI in archaeological molluscan shell assemblages: A Marshall Islands case study. Journal of Archaeological Science 57:168-179. https://doi.org/10.1016/j.jas.2015.01.017

Harrold, A.G., W.J.F. McDonald, M.S. Hopkins, J. Walker, C.S. Sandercoe and C.H. Thompson 1987 Studies in Landscape Dynamics in the Cooloola-Noosa River Area, Queensland. 5. Vascular Plants. CSIRO Division of Soils Divisional Report 89. Glen Osmond, SA: Commonwealth Scientific and Industrial Research Organisation.

Healy, J., M. Norman, D. Potter and R. Willan 2011 Molluscs. In P. Davie (ed.), Wild Guide to Moreton Bay and Adjacent Coasts, pp.119-196.Vol. 2. 2nd ed. Brisbane: Queensland Museum.

Healy, J.M. and D.G. Potter 2010 A preliminary checklist of the marine bivalves (Mollusca: Bivalvia) of Moreton Bay, Queensland. In P.J.F. Davie and J.A. Phillips (eds), Proceedings of the Thirteenth International Marine Biological Workshop, The Marine Fauna and Flora of Moreton Bay, Queensland. Memoirs of the Queensland Museum-Nature 54(3):235-252. Brisbane: Queensland Museum.

Heap, E.G. 1966 In the wake of the raftsmen: A survey of early settlement in the Maroochy district up to the passing of Macalister's Act (1868). Part III. Queensland Heritage 1(5):9-20.

Hekel, H., W.T. Ward, M. Jones and D.E. Searle 1979 Geological development of northern Moreton Bay. In A. Bailey and N.C. Stevens (eds), Northern Moreton Bay Symposium: Proceedings of a Symposium held at the Abel Smith Lecture Theatre, University of Queensland, September 23-24, 1978, pp.7-18. Brisbane: Royal Society of Queensland.

Hembrow, S.C., K.H. Taffs, P. Atahan, J. Parr, A. Zawadzki and H. Heijnis 2014 Diatom community response to climate variability over the past 37,000 years in the sub-tropics of the Southern Hemisphere. Science of the Total Environment 468-469:774-784. https://doi.org/ $\underline{10.1016 / j . s c i t o t e n v .2013 .09 .003}$

Hembrow, S.C., K.H. Taffs, P. Atahan, A. Zawadzki, H. Heijnis and J. Parr 2018 Mid-Holocene palaeolimnological record of a Southern Hemisphere subtropical lake spanning the last $\sim 6000$ years: Lake Jennings, Fraser Island, Australia. The Holocene 28(4):558-569. https://doi.org/10.1177\%2F09596836177355580

Hill, P.J. 1992 Capricorn and northern Tasman Basins: Structure and depositional systems. Exploration Geophysics 23(2):153-162. https://doi.org/10.1071/EG992153

Hiscock, P. 1985 The need for a taphonomic perspective in stone artefact analysis. Queensland Archaeological Research 2:82-97. https://doi.org/10.25120/qar.2.1985.197

Hofmann G.W. 1980 Quaternary sediments and geological history of the Pine Rivers area, southeast Queensland. Queensland Government Mining Journal 81:502-512. 
Hogg, A.G., Q. Hua, P.G. Blackwell, M. Niu, C.E. Buck, T.P. Guilderson, T.J. Heaton, J.G. Palmer, P.J. Reimer, R.W. Reimer, C.S. Turney and S.R.H. Zimmerman 2013 SHCal13 Southern Hemisphere calibration, 0-50,000 years cal BP. Radiocarbon 55(4):1889-1903. https://doi.org/10.2458/azu_is rc.55.16783

Howitt, A.W. 1904 The Native Tribes of South-East Australia. London: Macmillan and Co. Ltd.

Jerardino, A. 1997 Changes in shellfish species composition and mean shell size from a late-Holocene record of the west coast of southern Africa. Journal of Archaeological Science 24(11):10311044. https://doi.org/10.1006/jasc.1997.0182

Jerardino, A., G.M. Branch and R. Navarro 2008 Human impact on precolonial west coast marine environments of South Africa. In T.C. Rick and J.M. Erlandson (eds), Human Impacts on Ancient Marine Ecosystems: A Global Perspective, pp.279-296. Berkeley: Angeles: University of California Press.

Kamminga, J. 1981 The bevelled pounder: An Aboriginal stone tool type from southeast Queensland. Proceedings of the Royal Society of Queensland 92:31-36.

Kent, B.W. 1992 Making Dead Oysters Talk: Techniques for Analyzing Oysters from Archaeological Sites. Baltimore: Maryland Historical \& Cultural Publications for Maryland Historical Trust, Historic St. Mary's City, Jefferson Patterson Park and Museum.

Kerr, J. 1970 The Calooli Creek and Thannae railway, Tin Can Bay: Queensland's first private railway and first Queensland-built locomotive. Queensland Heritage 2(3):14-20.

Krull, E.S., C.H. Thompson and J.O. Skjemstad 2004 Chemistry, radiocarbon ages, and development of a subtropical acid peat in Queensland, Australia. Australian Journal of Soil Research 42(4):411-425. https://doi.org/10.1071/SR03144

Lam, K. and B. Morton 2006 Morphological and mitochondrialDNA analysis of the Indo-West Pacific rock oysters (Ostreidae: Saccostrea species). Journal of Molluscan Studies 72(3):235-245. https://doi.org/10.1093/mollus/eyl002

Lamprell, K.L. and J.M. Healy 1998 Bivalves of Australia. Volume 2. Leiden: Backhuys Publishers.

Lauer, P.K. 1977 Report on a preliminary ethnohistorical and archaeological survey of Fraser Island. In P.K. Lauer (ed.), Fraser Island, pp.1-38. Occasional Papers in Anthropology 8. St Lucia, QLD: Anthropology Museum, University of Queensland.

Lavers, H.J. 1871 A visit to the Aboriginal mission station, Fraser's Island. Maryborough Chronicle 29 August, p.3.

Leonard, N.D., K.J. Welsh, J-x. Zhao, L.D. Nothdurft, G.E. Webb, J. Major, Y. Feng and G.J. Price 2013 Mid-Holocene sea-level and coral reef demise: U-Th dating of subfossil corals in Moreton Bay, Australia. The Holocene 23(12):1841-1852. https://doi.org/ $10.1177 \% 2 \mathrm{~F} 0959683613508156$

Lewis, S.E., C.R. Sloss, C.V. Murray-Wallace, C.D. Woodroffe and S.G. Smithers 2013 Post-glacial sea-level changes around the Australian margin: A review. Quaternary Science Reviews 74:115138. https://doi.org/10.1016/j.quascirev.2012.09.006

Longmore, M. 1998 The mid-Holocene 'dry' anomaly on the mideastern coast of Australia: Calibration of palaeowater depth as a surrogate for effective precipitation using sedimentary loss on ignition in the perched lake sediments of Fraser Island, Queensland. Palaeoclimates: Data and Modelling 3(1-3):135-160.

Loyau, G.E. 1897 The History of Maryborough and Wide Bay and Burnett Districts from the Year 1850 to 1895. Brisbane: Pole, Outridge \& Co.

Lyman, R.L. 2008 Quantitative Paleozoology. Cambridge: Cambridge University Press.
Magurran, A.E. 2004 Measuring Biological Diversity. Oxford: Blackwell Publishing.

Major, J. 2012 Controls on Mid-Holocene Fringing Reef Growth and Termination in a High Latitude, Estuarine Setting, Wellington Point, Southeast Queensland. Unpublished Master of Applied Science thesis, Queensland University of Technology, Brisbane.

Mannino, M.A. and K.D. Thomas 2001 Intensive Mesolithic exploitation of coastal resources? Evidence from a shell deposit on the Isle of Portland (southern England) for the impact of human foraging on populations of intertidal rocky shore molluses. Journal of Archaeological Science 28(10):1101-1114. https://doi.org/ $\underline{10.1006 / j a s c .2001 .0658}$

Mannino, M.A. and K.D. Thomas 2002 Depletion of a resource? The impact of prehistoric human foraging on intertidal mollusc communities and its significance for human settlement, mobility and dispersal. World Archaeology 33(3):452-474. https://doi.org/ $\underline{10.1080 / 00438240120107477}$

Mason, R.D., M.L. Peterson and J.A. Tiffany 1998 Weighing vs. counting: Measurement reliability and the California School of midden analysis. American Antiquity 63(2):303-324. https://doi.org/ $\underline{10.2307 / 2694700}$

Mathew, J. 1910 Two Representative Tribes of Queensland. London: T. Fisher Unwin.

McIntyre, N. 1982 The Geological History and Sedimentology of a Tidal Inlet on a Sand Coastline, Southeast Queensland, Australia. Unpublished MSci thesis, Department of Geology and Mineralogy, University Queensland, Brisbane.

McKenzie, L.J. and S.J. Campbell 2003 Seagrass Resources of the Booral Wetlands and the Great Sandy Strait: February/March 2002. Cairns: Queensland Department of Primary Industries.

McKenzie, L.J., C. Collier and M. Waycott 2014 Reef Rescue Marine Monitoring Program: Inshore Seagrass, Annual Report for the Sampling Period 1st July 2011 - 31st May 2012. Cairns: TROPwater, James Cook University.

McNiven, I.J. 1984 Initiating Archaeological Research in the Cooloola Region, Southeast Queensland. Unpublished BA (Hons) thesis, Department of Anthropology and Sociology, University of Queensland, Brisbane.

McNiven, I.J. 1985 An archaeological survey of the Cooloola Region, S.E. Queensland. Queensland Archaeological Research 2:4-37. https://doi.org/10.25120/qar.2.1985.192

McNiven, I.J. 1989 Aboriginal shell middens at the mouth of the Maroochy River, southeast Queensland. Queensland Archaeological Research 6:28-52. https://doi.org/10.25120/qar.6.1989.136

McNiven, I.J. 1990a Prehistoric Aboriginal Settlement and Subsistence in the Cooloola Region, Coastal Southeast Queensland. Unpublished $\mathrm{PhD}$ thesis, Department of Anthropology and Sociology, The University of Queensland, Brisbane.

McNiven, I.J. 1990b Blowout taphonomy: Non-cultural associations between faunal and stone artefact assemblages along the Cooloola coast, southeast Queensland. Australian Archaeology 31:67-74. https://doi.org/10.1080/03122417.1990.11681390

McNiven, I.J. 1991a Settlement and subsistence activities along Tin Can Bay, southeast Queensland. Queensland Archaeological Research 8:85-107. https://doi.org/10.25120/qar.8.1991.119

McNiven, I.J. 1991b Teewah Beach: New evidence for Holocene coastal occupation in southeast Queensland. Australian Archaeology 33:14-27. https://doi.org/10.1080/03122417.1991.11681428

McNiven, I.J. 1991c The Double Island Point Aboriginal burials, coastal southeast Queensland. Australian Archaeology 32:10-16. https://doi.org/10.1080/03122417.1991.11681405 
McNiven, I.J. 1992a Ethnohistorical reconstructions of Aboriginal lifeways along the Cooloola coast, southeast Queensland. Proceedings of the Royal Society of Queensland 102:5-24.

McNiven, I.J. 1992b Bevel-edged tools from coastal southeast Queensland. Antiquity 66(252):701-709. https://doi.org/10.1017/ $\underline{\text { S0003598X00039405 }}$

McNiven, I.J. 1992c Shell middens and mobility: The use of off-site faunal remains, Queensland, Australia. Journal of Field Archaeology 19(4):495-508.

McNiven, I.J. 1994 Booral: Cultural Heritage Management Plan. Unpublished report to the Queensland Department of Environment and Heritage, Maryborough.

McNiven, I.J. 1999 Fissioning and regionalisation: The social dimensions of changes in Aboriginal use of the Great Sandy Region, southeast Queensland. In J. Hall and I.J. McNiven (eds), Australian Coastal Archaeology, pp.157-168. Canberra: Archaeology and Natural History Publications, Research School of Pacific and Asian Studies, Australian National University.

McNiven, I.J. 2006 Late moves on Donax: Aboriginal marine specialisation in southeast Queensland over the last 6000 years. In S. Ulm and I. Lilley (eds), An Archaeological Life: Papers in Honour of Jay Hall, pp.109-124. Brisbane: Aboriginal and Torres Strait Islander Studies Unit, The University of Queensland.

Meehan, B. 1982 Shell Bed to Shell Midden. Canberra: Australian Institute of Aboriginal Studies.

Meston, A. 1895 Geographic History of Queensland. Brisbane: Government Printer.

Meston, A. 1923 The Dora Dora black: How it happened. World's News 7 July, p. 12.

Miller, O. 1993 Fraser Island Legends. Milton, QLD: The Jacaranda Press.

Milner, N. 2013 Human impacts on oyster resources at the Mesolithic-Neolithic transition in Denmark. In V.D. Thompson and J.C. Waggoner (eds), The Archaeology and Historical Ecology of Small Scale Economies, pp.17-40. Gainsville, FL: University Press of Florida.

Milner, N., J. Barrett and J. Welsh 2007 Marine resource intensification in Viking Age Europe: The molluscan evidence from Quoygrew, Orkney. Journal of Archaeological Science 34(9):14611472. https://doi.org/10.1016/j.jas.2006.11.004

Moreton, D. and A. Ross 2011 Gorenpul-Dandrabin Knowledge. In P. Davie (ed.), Wild Guide to Moreton Bay and Adjacent Coasts, pp.59-67. Vol. 1. 2nd ed. Brisbane: Queensland Museum.

Morrison, M. 2003 Old boundaries and new horizons: The Weipa shell mounds reconsidered. Archaeology in Oceania 38(1):1-8. https://doi.org/10.1002/j.1834-4453.2003.tb00516.x

Morrison, M. 2015 Late Holocene Aboriginal shellfish production strategies in northern Australia: Insights from Prunung (Red Beach), Weipa, Cape York Peninsula. Queensland Archaeological Research 18:1-27. https://doi.org/10.25120/qar.18.2015.3498

Morton, R.M. and T. Healy 1992 Fisheries Resources of the Fraser Island Region. Brisbane: Queensland Fisheries Management Authority.

Morwood, M.J. 1975 Report on a Preliminary Archaeological Survey of Fraser Island Conducted 19.4.75. Unpublished report to Cultural Heritage Branch, Queensland Department of Environment and Heritage, Brisbane.

Morwood, M.J. 1987 The archaeology of social complexity in southeast Queensland. Proceedings of the Prehistoric Society 53:337350. https://doi.org/10.1017/S0079497X00006265

Moss, P., A. Dudgeon, F. Shapland, L. Withers, C. Brownhall, D. Terzano, L. Petherick and C. Sloss 2013 Investigation into the
Vegetation and Fire History of the EPBC, Ramsar and WHA Wetlands of the Great Sandy Straits, South East Queensland. Unpublished report to Burnett Mary Regional Group, Bundaberg, Queensland.

Moss, P.T., J. Tibby, F. Shapland, R. Fairfax, P. Stewart, C. Barr, L. Petherick, A. Gontz and C. Sloss 2016 Patterned fen formation and development from the Great Sandy Region, south-east Queensland, Australia. Marine and Freshwater Research 67(6):816-827. https://doi.org/10.1071/MF14359

Murray-Jones, S. 1999 Conservation and Management in Variable Environments: The Surf Clam, Donax deltoides. Unpublished PhD thesis, Department of Biological Sciences, University of Wollongong, Wollongong.

Nique, P. and W. Hartenstein 1841 The Aborigines. Diary of Messrs. Nique and Hartenstein, of the German Mission to the Aborigines, at Moreton Bay, during a journey to Toorbal, a district of country to the Northward. The Colonial Observer 28 October, p.27.

Nolan, A. 1986 Sandstone Point: Temporal and Spatial Patterns of Aboriginal Site Use at a Midden Complex, South-East Queensland. Unpublished BA (Hons) thesis, Department of Anthropology and Sociology, The University of Queensland, Brisbane.

Nunn, P.D., R. Hunter-Anderson, M.T. Carson, F. Thomas, S. Ulm and M.J. Rowland 2007 Times of plenty, times of less: Lastmillennium societal disruption in the Pacific Basin. Human Ecology 35(4):385-401. https://doi.org/10.1007/s10745-006-9090-5

Pallant, J. 2013 SPSS Survival Manual: A Step by Step Guide to Data Analysis using SPSS. 5th ed. Sydney: Allen \& Unwin Publishing.

Payenberg, T.H.D., R. Boyd, J. Beaudoin, K. Ruming, S. Davies, J. Roberts and S.C. Lang 2006 The filling of an incised valley by shelf dunes - An example from Hervey Bay, east coast of Australia. In R.W. Dalrymple, D.A. Leckieand and R.W. Tillman (eds), Incised Valleys in Time and Space, pp.87-98. Society for Sedimentary Geology (SEPM) Special Publication 85. https://doi.org/10.2110/ pec. 06.85 .0087

Petrie, C.C. 1904 Tom Petrie's Reminiscences of Early Queensland. Brisbane: Watson, Ferguson and Co.

Petrie, W.R. 1921 A Note on "Dundathu" Kauri (Agathis robusta). Forestry Bulletin 4. Queensland Forest Services, Department of Public Lands, Queensland. Brisbane: Government Printer.

Preen, A. and H. Marsh 1995 Response of dugongs to large-scale loss of seagrass from Hervey Bay, Queensland Australia. Wildlife Research 22(4):507-519. https://doi.org/10.1071/WR9950507

QBPA (Queensland Beach Protection Authority) 1989 Hervey Bay Beaches: A Detailed Study of Coastline Behaviour along the Mainland Beaches of Hervey Bay, South-East Queensland, Australia. Brisbane: Queensland Beach Protection Authority.

Reeve, R., I.F. Fergus and C.H. Thompson 1985 Studies in Landscape Dynamics in the Cooloola-Noosa River Area, Queensland. 4. Hydrology and Water Chemistry. CSIRO Division of Soils Divisional Report 77.

Reeves, J.M., T.T. Barrows, T.J. Cohen, A.S. Kiem, H.C. Bostock, K.E. Fitzsimmons, J.D. Jansen, J. Kemp, C. Krause, L. Petherick and S.J. Phipps 2013 Climate variability over the last 35,000 years recorded in marine and terrestrial archives in the Australian region: An OZ-INTIMATE compilation. Quaternary Science Reviews 74:21-34. https://doi.org/10.1016/j.quascirev.2013.01.001

Reeves, W. and O. Miller 1964 The Legends of Moonie Jarl. Brisbane: Jacaranda Press.

Reimer, P.J., E. Bard, A. Bayliss, J.W. Beck, P.G. Blackwell, C. Bronk Ramsey, C.E. Buck, H. Cheng, R.L. Edwards, M. Friedrich, P.M. Grootes, T.P. Guilderson, H. Haflidason, I. Hajdas, C. Hatté, T.J. Heaton, D.L. Hoffman, A.G. Hogg, K.A. Hughen, K.F. Kaiser, B. Kromer, S.W. Manning, M. Niu, R.W. Reimer, D.A. Richards, 
E.M. Scott, J.R. Southon, R.A. Staff, C.S.M. Turney and J. van der Plicht 2013 IntCal13 and Marine13 radiocarbon age calibration curves 0-50,000 years cal BP. Radiocarbon 55(4):1869-1887. https://doi.org/10.2458/azu is rc. 55.16947

Reitz, E.J. and E.S. Wing 2008 Zooarchaeology. Cambridge: Cambridge University Press.

Rick, T.C. and J.M. Erlandson 2008 Archaeology, historical ecology, and the future of the world's oceans. In T.C. Rick and J.M. Erlandson (eds), Human Impacts on Ancient Marine Ecosystems: A Global Perspective, pp.297-308. Berkeley: University of California Press.

Rick, T.C., J.M. Erlandson and R.L. Vellanoweth 2001 Paleocoastal marine fishing on the Pacific coast of the Americas: Perspectives from Daisy Cave, California. American Antiquity 66(4):595-613. https://doi.org/10.2307/2694175

Rick, T.C., J.M. Erlandson, R.L. Vellanoweth and T.J. Braje 2005 From Pleistocene mariners to complex hunter-gatherers: The archaeology of the California Channel Islands. Journal of World Prehistory 19(3):169-228. https://doi.org/10.1007/s10963-0069004-x

Robins, R.P. and E.C. Stock 1990 The burning question: A study of molluscan remains from a midden on Moreton Island. In S. Solomon, I. Davidson and D. Watson (eds), Problem Solving in Taphonomy: Archaeological and Palaeontological Studies from Europe, Africa and Oceania, pp.80-100. Tempus 2. St Lucia, QLD: Anthropology Museum, University of Queensland.

Robins, R., J. Hall and E. Stock 2015 Geoarchaeology and the archaeological record in the coastal Moreton Region, Queensland, Australia. Quaternary International 385:191-205. https://doi.org/ 10.1016/j.quaint.2015.02.060

Ross, A., and R. Duffy 2000 Fine mesh screening of midden material and the recovery of fish bone: The development of flotation and deflocculation techniques for an efficient and effective procedure. Geoarchaeology 15(1):21-41. https://doi.org/10.1002/(SICI)15206548(200001)15:1\%3C21::AID-GEA2\%3E3.0.CO;2-L

Ross, A. and H. Tompkins 2011 Fishing for data-The value of finemesh screening for fish-bone recovery: A case study from Peel Island, Moreton Bay, Queensland. Technical Reports of the Australian Museum 23:133-145. https://doi.org/10.3853/j.18354211.23.2011.1574

Rowland, M.J. 1994 Size isn't everything: Shells in mounds, middens and natural deposits. Australian Archaeology 39:118-124. https://doi.org/10.1080/03122417.1994.11681536

Russell, H.S. 1845 Exploring excursions in Australia. The Journal of the Royal Geographical Society of London 15:305-327.

Russell, H.S. 1888 The Genesis of Queensland. Sydney: Turner \& Henderson.

Ryan, D.A., A.D. Heap, L. Radke and D. T. Heggie 2003 Conceptual Models of Australia's Estuaries and Coastal Waterways: Applications for Coastal Resource Management. Canberra: Geoscience Australia, Department of Industry, Tourism and Resources.

Sandercoe, C. 1986 Womalah State Forest and Part of the Cooloola National Park Map $(1: 25,000)$. 1st ed. Brisbane: Department of Forestry.

Satterthwait, L. and A. Heather 1987 Determinants of earth circle site location in the Moreton region, southeast Queensland. Queensland Archaeological Research 4:5-53. http://dx.doi.org/ 10.25120/qar.4.1987.171

Sloss, C.R., L. Nothdurft, Q. Hua, S.G. O’Connor, P.T. Moss, D. Rosendahl, L.M. Petherick, R.A. Nanson, L.L. Mackenzie, A. Sternes, G.E. Jacobsen and S. Ulm 2018 Holocene sea-level change and coastal landscape evolution in the southern Gulf of Carpentaria,
Australia. The Holocene 28(9):1411-1430. https://doi.org/ $10.1177 \% 2 \mathrm{~F} 0959683618777070$

Smith, A.D. 1992 An Archaeological Site Location and SubsistenceSettlement Analysis of Bribie Island, Southeast Queensland. Unpublished BA (Hons) thesis, Department of Anthropology and Sociology, University of Queensland, Brisbane.

Smith, A.D. 2003 Archaeological Spatial Variability on Bribie Island, Southeast Queensland. Unpublished MA thesis, School of Social Science, University of Queensland, Brisbane.

Smith, A.D. 2016 Archaeological Expressions of Holocene Cultural and Environmental Change in Coastal Southeast Queensland. Unpublished $\mathrm{PhD}$ thesis, School of Social Science, University of Queensland, Brisbane.

Smith, G.S. 1981 Southern Queensland oyster industry. Journal of the Royal Historical Society of Queensland 11(3):45-58.

Smith, G.S. 1985 The Queensland Oyster Fishery: An Illustrated History. Brisbane: Queensland Department of Primary Industries.

Steele, J.G. 1983 Aboriginal Pathways in Southeast Queensland and the Richmond River. St Lucia, QLD: University of Queensland Press.

Stockton, J. 1974 Report of an Archaeological Survey in the Vicinity of Bribie Island, Southeast Queensland. Unpublished BA (Hons) thesis, Department of Anthropology and Sociology, University of Queensland, Brisbane.

Stuiver, M. and P.J. Reimer 1993 Extended 14C data base and revised CALIB $3.014 \mathrm{C}$ calibration program. Radiocarbon 35(1):215-230. https://doi.org/10.1017/S0033822200013904

Szabó, K.A. 2009 Molluscan remains from Fiji. In G. Clark and A. Anderson (eds), The Early Prehistory of Fiji, pp.183-212. Canberra: ANU E Press.

The Vagabond 1886 Fraser Island and the Wide Bay district, Queensland. Australasian Sketcher with Pen and Pencil 13 January, p.10.

Thomson, J.M. 1954 The genera of oysters and the Australian species. Australian Journal of Marine and Freshwater Research 5(1):132-168. https://doi.org/10.1071/MF9540132

Thompson, C.H. and A.W. Moore 1984. Studies in Landscape Dynamics in the Cooloola-Noosa River Area, Queensland. 1. Introduction, General Description and Research Approach. CSIRO Division of Soils Divisional Report 73.

Thompson, V.D. 2013 Whispers on the Landscape. In V.D. Thompson and J.C. Waggoner Jr (eds), The Archaeology and Historical Ecology of Small Scale Economies, pp.1-13. Gainsville, FL: University Press of Florida. https://doi.org/10.5744/ florida/9780813042428.003.0001

Thorne, E. 1876 The Queen of the Colonies; or, Queensland as I Knew It. London: Sampson Low, Marston, Searle, and Rivington.

Tindale, N.B. 1974 Aboriginal Tribes of Australia. Berkeley: University of California Press.

Ulm, S., 2002a Marine and estuarine reservoir effects in central Queensland, Australia: determination of $\Delta \mathrm{R}$ values. Geoarchaeology 17(4):319-348. https://doi.org/10.1002/gea.10017

Ulm, S. 2002b Reassessing marine fishery intensification in southeast Queensland. Queensland Archaeological Research 13:7996. https://doi.org/10.25120/qar.13.2002.70

Ulm, S. 2006a Coastal Themes: An Archaeology of the Southern Curtis Coast, Queensland. Terra Australis 24. Canberra: ANU E Press.

Ulm, S. 2006b Australian marine reservoir effects: A guide to $\Delta \mathrm{R}$ values. Australian Archaeology 63:57-60. https://doi.org/10.1080/ $\underline{03122417.2006 .11681838}$

Queensland Archaeological Research | Vol. 22 | 2019 | 37 
Ulm, S. 2011 Coastal foragers on southern shores: Marine resource use in northeast Australia since the late Pleistocene. In N.F. Bicho, J.A. Haws and L.G. Davis (eds), Trekking the Shore: Changing Coastlines and the Antiquity of Coastal Settlement, pp.441-461. Springer: New York. https://doi.org/10.1007/978-1-4419-8219$\underline{3 \_19}$

Ulm, S. and J. Hall 1996 Radiocarbon and cultural chronologies in south Queensland prehistory. In S. Ulm, I. Lilley and A. Ross (eds), Australian Archaeology '95: Proceedings of the 1995 Australian Archaeological Association Annual Conference, pp.45-62. Tempus 6. St Lucia, QLD: University of Queensland Anthropology Museum.

Ulm, S., F. Petchey and A. Ross 2009 Marine reservoir corrections for Moreton Bay, Australia. Archaeology in Oceania 43(3):160-166. https://doi.org/10.1002/j.1834-4453.2009.tb00060.x

Vale, D. and R.H. Gargett 2002 Size matters: 3-mm sieves do not increase richness in a fishbone assemblage from Arrawarra I, an Aboriginal Australian shell midden on the mid-north coast of New South Wales, Australia. Journal of Archaeological Science 29(1):57-63. https://doi.org/10.1006/jasc.2001.0704

Viator 1879 Dugong fishing in Queensland. - No VII. - "The Aborigines." Leader 24 May, p.1.

Vohra, F.C. 1965 Ecology of Intertidal Zostera Flats of Moreton Bay. Unpublished PhD thesis, Department of Zoology, University of Queensland, Brisbane.

Walker, J., B. Lees, J. Olley and C. Thompson 2018 Dating the Cooloola coastal dunes of south-eastern Queensland, Australia. Marine Geology 398:73-85. https://doi.org/10.1016/j.margeo. $\underline{2017.12 .010}$

Walters, I. 1984 Gone to the Dogs: a Study of Bone Attrition at a Central Australian Campsite. Mankind 14(5):389-400. https://doi.org/10.1111/j.1835-9310.1984.tb01265.x

Walters, I. 1989 Intensified fishery production at Moreton Bay, southeast Queensland, in the late Holocene. Antiquity 63(239):215224. https://doi.org/10.1017/S0003598X00075943

Walters, I. 1992 Farmers and their fires, fishers and their fish: Production and productivity in pre-European south-east Queensland. Dialectical Anthropology 17(2):167-182. https://doi.org/ $\underline{10.1007 / B F 00258089}$

Walters, I., P. Lauer, A. Nolan, G. Dillon and M. Aird 1987 Hope Island: Salvage excavation of a Kombumerri site. Queensland Archaeological Research 4:80-95. https://doi.org/10.25120/ qar.4.1987.173

Ward, W.T. and K.G. Grimes 1987 History of coastal dunes at Triangle Cliff, Fraser Island, Queensland. Australian Journal of Earth Sciences 34(3):325-333. https://doi.org/10.1080/ $\underline{08120098708729414}$

Ward, W.T. and J.L.F. Hacker 2006 Brisbane Airport: An alluvial landscape veiled by marine sediments. Australian Journal of Earth Sciences 53(6):1001-1012. $\quad$ https://doi.org/10.1080/ $\underline{08120090600880933}$

Watson, F.J. 1946 Vocabularies of Four Representative Tribes of South Eastern Queensland. Supplement to Journal of the Royal Geographical Society of Australasia (Queensland) 34(48).
Williams, A.N., S. Ulm, T. Sapienza, S. Lewis and C.S.M. Turney 2018 Sea-level change and demography during the last glacial termination and early Holocene across the Australian continent. Quaternary Science Reviews 182:144-154. https://doi.org/ $\underline{10.1016 / \text { j.quascirev.2017.11.030 }}$

Wilson, B.R. 1993 Australian Marine Shells. Vol. 1 \& 2. Kallaroo, WA: Odyssey Publishing.

Withers, L. 2013 Palaeoenvironmental Reconstructions of a Subtropical Empodisma minus Wetland Comprising of Extensive Areas of Patterned Fen: The Great Sandy Region, Southeast Queensland. Unpublished Honours thesis, School of Geography, Planning and Environmental Management, University of Queensland, Brisbane.

Woltering, M., P. Atahan, K. Grice, H. Heijnis, K. Taffs and J. Dodson 2014 Glacial and Holocene terrestrial temperature variability in subtropical east Australia as inferred from branched GDGT distributions in a sediment core from Lake McKenzie. Quaternary Research 82(1):132-145. https://doi.org/10.1016/ j.yqres.2014.02.005

Wood, P.A. 1972 A possible Holocene shoreline at Maroochydore, Queensland. Queensland Government Mining Journal 73(850):331.

Wood, P.A. and H. King 1974 A Holocene molluscan fauna from Maroochydore, Queensland. Memoirs of the Queensland Museum 17(1):69-71.

Woolley, L.M. 2016 Histories of the Great Sandy Straits. Pukpuk Publications.

Zuschin, M. and R.J. Stanton Jr 2001 Experimental measurement of shell strength and its taphonomic interpretation. Palaios 16(2):161170. $\quad$ https://doi.org/10.1669/0883-1351(2001)016\%3C0161 :EMOSSA\%3E2.0.CO;2

Citation: Smith, T. and I.J. McNiven 2019 Aboriginal marine subsistence foraging flexibility in a dynamic estuarine environment: The late development of Tin Can Inlet (southeast Queensland) middens revisited. Queensland Archaeological Research 22:1-38. https://doi.org/10.25120/qar.22.2019.3670 